\title{
Architectural Organization of the African Elephant Diencephalon and Brainstem
}

\author{
Busisiwe C. Maseko ${ }^{a}$ Nina Patzke ${ }^{a}$ Kjell Fuxe ${ }^{b}$ Paul R. Manger ${ }^{a}$ \\ ${ }^{a}$ School of Anatomical Sciences, Faculty of Health Sciences, University of the Witwatersrand, Johannesburg, \\ Republic of South Africa; ${ }^{b}$ Department of Neuroscience, Karolinska Institutet, Stockholm, Sweden
}

\author{
Key Words \\ Elephant $\cdot$ Dorsal thalamus $\cdot$ Epithalamus $\cdot$ Hypothalamus . \\ Midbrain · Pons · Medulla oblongata $\cdot$ Proboscidae $\cdot$ Brain \\ evolution · Mammalia
}

\begin{abstract}
The current study examined the organization of the diencephalon and brainstem of the African elephant (Loxodonta africana) - a region of the elephant brain that has not been examined for at least 50 years. The current description, employing material amenable for use with modern neuroanatomical methods, shows that, for the most part, the elephant diencephalon and brainstem are what could be considered typically mammalian, with subtle differences in proportions and topology. The variations from these previous descriptions, where they occurred, were related to four specific aspects of neural information processing: (1) the motor systems, (2) the auditory and vocalization systems, (3) the orexinergic satiety/wakefulness centre of the hypothalamus and the locus coeruleus, and (4) the potential neurogenic lining of the brainstem. For the motor systems, three specific structures exhibited interesting differences in organization - the pars compacta of the substantia nigra, the facial motor nerve nucleus, and the inferior olivary nuclear complex, all related to the timing and learning of movements and likely related to the control of the trunk. The dopaminergic neurons of the substantia nigra appear to form distinct islands separated from each other by large fibre pathways, an appearance
\end{abstract}

unique to the elephant. Each island may send topologically organized projections to the striatum forming a dopaminergic innervation mosaic that may relate to trunk movements. At least five regions of the combined vocalization production and auditory/seismic reception system were specialized, including the large and distinct nucleus ellipticus of the periaqueductal grey matter, the enlarged lateral superior olivary nucleus, the novel transverse infrageniculate nucleus of the dorsal thalamus, the enlarged dorsal column nuclei and the ventral posterior inferior nucleus of the dorsal thalamus. These specializations, related to production and reception of infrasound, allow the proposal of a novel concept regarding the reception and localization of infrasonic sources. The orexinergic system of the hypothalamus displayed a medial hypothalamic parvocellular cluster of neurons in addition to the magnocellular clusters typical of mammals located in the lateral hypothalamus, and a novel medial division of the locus coeruleus was observed in the pons. These systems are related to appetitive drive and promotion of wakefulness, two aspects of elephant behaviour that appear to be inextricably linked. Lastly, we observed an extensive potential neurogenic lining of the ventricles throughout the brainstem that is present in even quite old elephants, although the function of these cells remains elusive. These observations combined demonstrate that, while much of the elephant brainstem is typically mammalian, certain aspects of the anatomy related to specialized behaviour of elephants are present and instructive in understanding elephant behaviour.

(c) 2013 S. Karger AG, Basel

\begin{tabular}{ll}
\hline KARGER & $\begin{array}{l}\text { ( } 2013 \text { S. Karger AG, Basel } \\
0006-8977 / 13 / 0822-0083 \$ 38.00 / 0\end{array}$ \\
$\begin{array}{l}\text { E-Mail karger@karger.com } \\
\text { www.karger.com/bbe }\end{array}$ & $\begin{array}{l}\text { This is an Open Access article licensed under the terms of the } \\
\text { Creative Commons Attribution-NonCommercial 3.0 Un- } \\
\text { ported license (CC BY-NC) (www.karger.com/OA-license), } \\
\text { applicable to the online version of the article only. Distribu- } \\
\text { tion permitted for non-commercial purposes only. }\end{array}$
\end{tabular}

Paul Manger

School of Anatomical Sciences, Faculty of Health Sciences

University of the Witwatersrand, 7 York Road

Parktown, Johannesburg 2193 (Republic of South Africa)

E-Mail Paul.Manger@wits.ac.za 


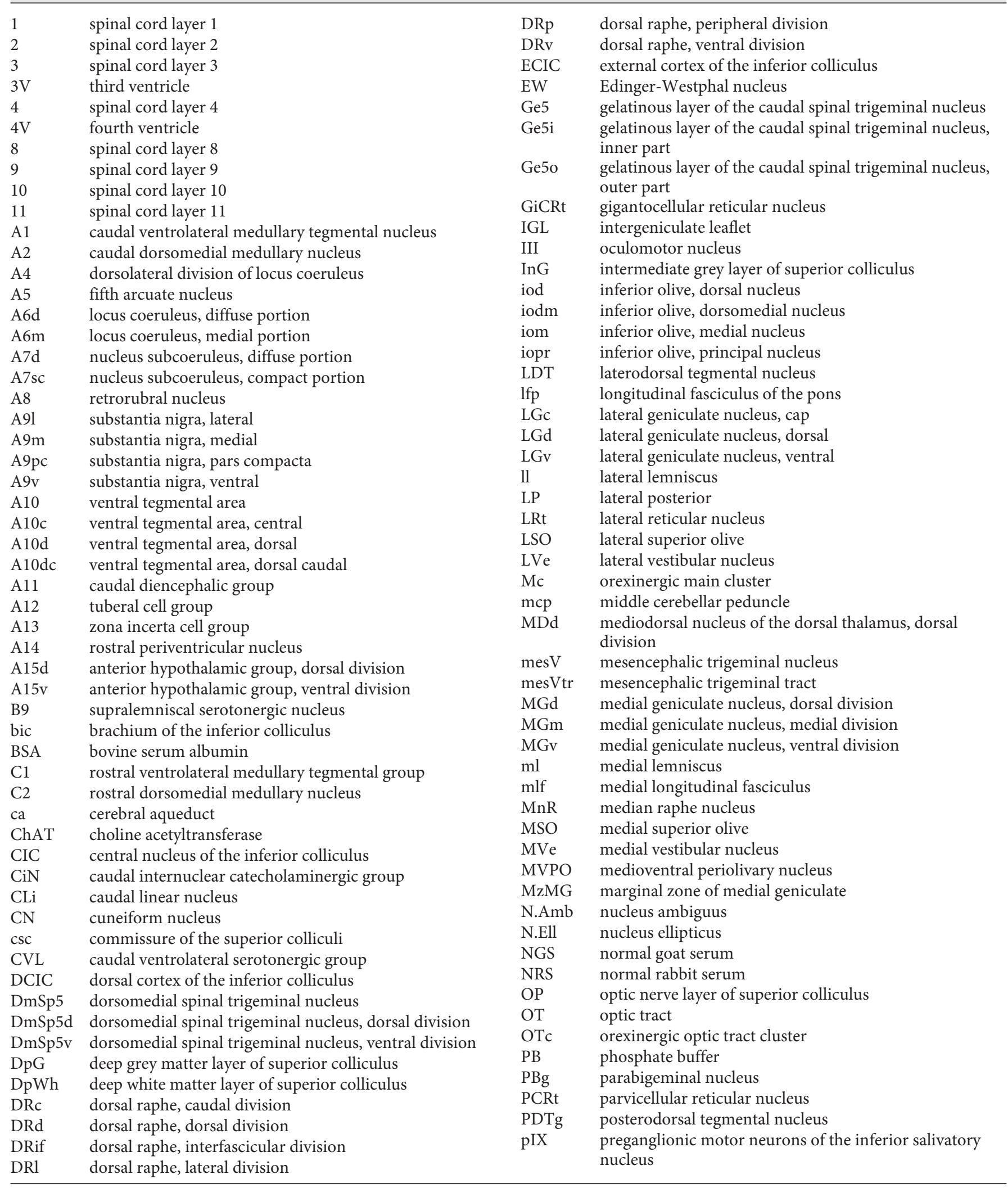




\begin{tabular}{|c|c|}
\hline \multicolumn{2}{|c|}{ Abbreviations used in this paper } \\
\hline PPT & pedunculopontine tegmental nucleus \\
\hline PrVdm & principal trigeminal nucleus, dorsomedial \\
\hline PrVvl & principal trigeminal nucleus, ventrolateral \\
\hline PV & paraventricular thalamic nuclei \\
\hline pVII & $\begin{array}{l}\text { preganglionic motor neurons of the superior salivatory } \\
\text { nucleus or facial nerve }\end{array}$ \\
\hline $\mathrm{RMg}$ & raphe magnus nucleus \\
\hline $\mathrm{ROb}$ & raphe obscurus nucleus \\
\hline $\mathrm{RPa}$ & raphe pallidus nucleus \\
\hline RtTg & reticulotegmental nucleus of the pons \\
\hline RVL & rostral ventrolateral serotonergic group \\
\hline scp & superior cerebellar peduncle \\
\hline Sp5 & spinal trigeminal tract \\
\hline $\mathrm{Sp} 5 \mathrm{c}$ & spinal trigeminal nucleus, caudal part \\
\hline Sp5l & spinal trigeminal nucleus, lateral part \\
\hline Sp5o & spinal trigeminal nucleus, oral part \\
\hline SPO & superior paraolivary nucleus \\
\hline $\mathrm{SuG}$ & superficial grey matter of superior colliculus \\
\hline TIN & transverse infrageniculate nucleus \\
\hline VA & ventral anterior nucleus of the dorsal thalamus \\
\hline $\mathrm{VeCb}$ & vestibulocerebellar nucleus \\
\hline VIIdm & facial nerve nucleus, dorsomedial division \\
\hline VIIdv & facial nerve nucleus, dorsoventral division \\
\hline VIIv & facial nerve nucleus, ventral division \\
\hline VIIvm & facial nerve nucleus, ventromedial division \\
\hline & Vmb basal ventromedial nucleus of the dorsal thalamus \\
\hline Vmot & motor nucleus of the trigeminal nerve \\
\hline VPI & ventral posterior inferior nucleus of the dorsal thalamus \\
\hline VPL & principal ventrolateral nucleus of the thalamus \\
\hline VPM & principal ventromedial nucleus of the thalamus \\
\hline VPO & ventral pontine nucleus \\
\hline VTA & ventral tegmental area \\
\hline $\mathrm{X}$ & dorsal motor vagus nucleus \\
\hline XII & hypoglossal nucleus \\
\hline $\mathrm{xscp}$ & decussation of the superior cerebellar peduncle \\
\hline Zic & orexinergic zona incerta cluster \\
\hline Zo & zonal layer of the superior colliculus \\
\hline
\end{tabular}

\section{Introduction}

The gross anatomical appearance of the diencephalon and brainstem of the elephant was recently detailed by Shoshani et al. [2006]. Shoshani et al. [2006] noted that the general appearance of the elephant diencephalon is not markedly different from that of the human one, although it appears relatively large. Visible medial and lateral geniculate bodies in the dorsal thalamus, a distinct hypothalamic sulcus delimiting the dorsal thalamus from the hypothalamus, a very large massa intermedia thalami, and relatively small and flattened mammillary bodies were noted. In a histological study of the elephant diencephalon, Abe [1952] noted that the various subnuclei and anatomical divisions of the dorsal thalamus were indistinct, also noting that the internal medullary lamina was difficult to discern. The one feature of the dorsal thalamus that appears to be specific to the elephant is the presence of a laminated transverse infrageniculate nucleus located ventral and lateral to the medial geniculate body [Precechtel, 1925; Abe, 1952]. The hypothalamus also appears to have the typical mammalian morphology [Diepen et al., 1956].

Describing the gross anatomical appearance of the elephant brainstem, Shoshani et al. [2006] noted the standard vertebrate subdivisions of midbrain (mesencephalon), pons (metencephalon) and medulla oblongata (myelencephalon). In each of these subdivisions, the majority of structures visible to the naked eye in general gross anatomical dissections of mammalian brains, including the corpora quadrigemina, the cerebral aqueduct, the central grey matter, red nuclei, substantia nigra, cerebral peduncles, cerebellar peduncles, cranial nerves and their nuclei, the median sulcus, hypoglossal and vagal trigones, pyramids, and olives, were observed. Specifically in the elephant, Shoshani et al. [2006] noted the presence of the nucleus ellipticus in the midbrain, as previously described [Precechtel, 1925], the relatively flat and narrow appearance of the pons, and the large and flattened pyramidal tracts [Verhaart and Kramer, 1958; Verhaart, 1962, 1963]. Despite these observations, there appears to be nothing specific within the elephant brainstem that has not been observed in other mammals.

While substantial data regarding the basic organization of the elephant diencephalon and brainstem has been provided, much of this data is geared towards observations of the gross anatomical aspects of the brain [e.g. Shoshani et al., 2006] or were undertaken at a time, and on specimens, where the range of neuroanatomical stains that could be applied to the tissue was limited [Precechtel, 1925; Abe, 1952; Diepen et al., 1956; Verhaart, 1962, 1963; reviewed in Cozzi et al., 2001]. The acquisition of African elephant tissue that was perfusion-fixed with paraformaldehyde following a short autolysis time [Manger et al., 2009] has provided the opportunity to re-examine the anatomy of the elephant diencephalon and brainstem using a range of neuroanatomical stains, including several immunohistochemical stains, to reveal specific neuronal populations. The burgeoning understanding of the structure and function of the mammalian diencephalon and brainstem [e.g. Klemm and Vertes, 1990; Jones, 2007], along with tissue that can be processed with modern neuroanatomical techniques, permits a detailed re-examination of the structure of these regions of the elephant brain. 


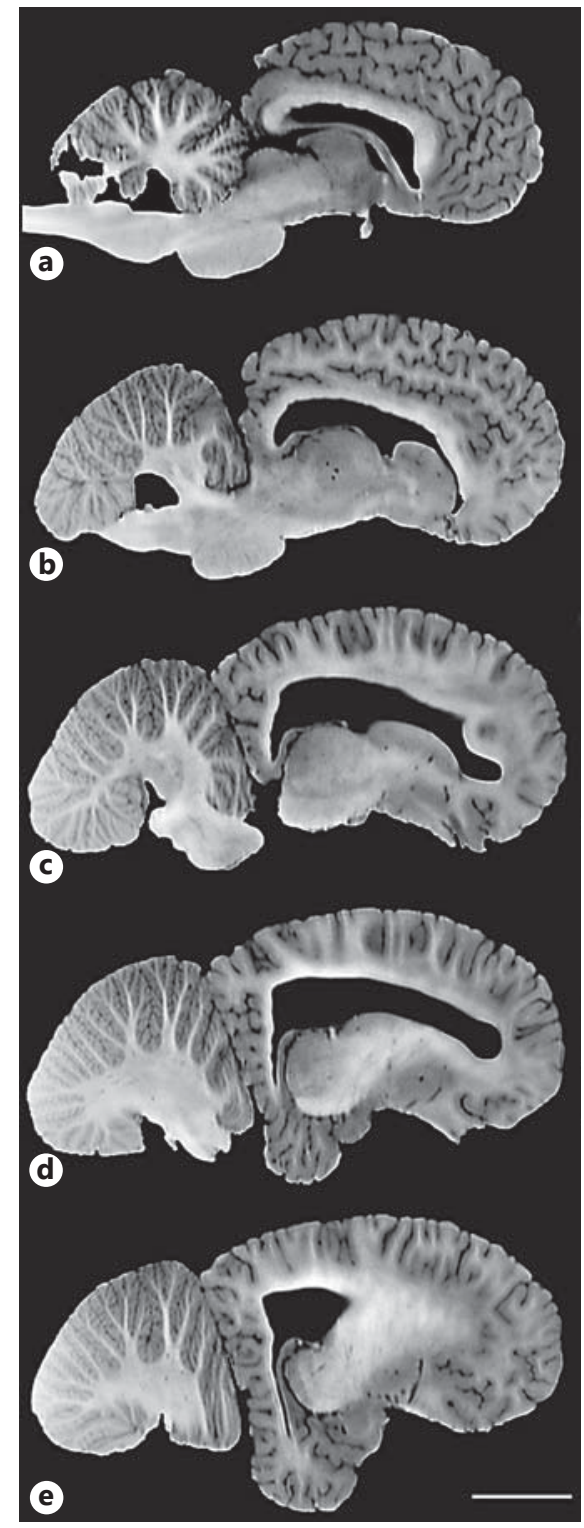

Fig. 1. MR images of a series of sagittal sections (a being the most medial and e being the most lateral) showing the general structure of the diencephalon and brainstem of the specimen (LA1) sectioned and stained in the current study. Each section is approximately $10 \mathrm{~mm}$ apart. Scale bar $=5 \mathrm{~cm}$.

Given the important role these regions of the brain will play in the life history of the elephant, for example in control of the musculature of the trunk or the muscles controlling the production of infrasound [Endo et al., 2001; Herbst et al., 2012], a better understanding of the anatomy of the elephant diencephalon and brainstem may lead to greater insights into these enigmatic mammals. The
African elephant belongs to the supercohort Afrotheria, which includes the manatees and dugongs, hyraxes, elephant shrews, tenrecs, golden moles and aardvarks [Tabuce et al., 2008]. Morevoer, the Afrotheria are part of the Atlantogenata, which form a group of mammals distinct to the Boreoeutheria, which includes primates, carnivores and rodents among others [Asher et al., 2009]; thus, studies of the Afrotheria and Atlantogenata are important for our understanding of the organization of the ancestral Eutherian brain.

\section{Materials and Methods}

The brains of three adult male African elephants (Loxodonta africana) were acquired following a planned cull in Southeastern Zimbabwe. The animals were sacrificed and perfused in the field under the permission and supervision of the Zimbabwe Parks and Wildlife Management Authority [Manger et al., 2009]. All animals were treated and used according to the guidelines of the University of Witwatersrand Animal Ethics Screening Committee (AESC), which parallel those of the NIH for the care and use of animals in scientific experimentation [see Manger et al., 2009, for a detailed account of the acquisition of elephant brains]. The head of each elephant was perfused through the carotid arteries initially with a rinse of 100 liters of $0.9 \%$ saline, followed by fixation with 100 liters of $4 \%$ paraformaldehyde in $0.1 \mathrm{M}$ phosphate buffer (PB). The brains were then removed from the skull and post-fixed (in $4 \%$ paraformaldehyde in $0.1 \mathrm{M} \mathrm{PB}$ ) for $72 \mathrm{~h}$ at $4^{\circ} \mathrm{C}$. Thereafter, the brains were transferred to a solution of $30 \%$ sucrose in $0.1 \mathrm{M} \mathrm{PB}$ at $4{ }^{\circ} \mathrm{C}$ until they had equilibrated and were then transferred to an antifreeze solution containing 30\% glycerol, 30\% ethylene glycol, $30 \%$ distilled water and $10 \% 0.244 \mathrm{M} \mathrm{PB}$. Once again the brains were allowed to equilibrate in the solution at $4^{\circ} \mathrm{C}$ and were then moved to the $-20^{\circ} \mathrm{C}$ freezer for storage until sectioning. Prior to sectioning, all three brains underwent MR imaging (fig. 1) to reveal the general anatomy of the diencephalon and brainstem of the elephant [full MRI protocol previously described in Manger et al., 2010, 2012; Maseko et al., 2011, 2012]. As all three specimens showed a similar anatomy (i.e. there was no obvious variance between the three specimens), we selected a single specimen (LA1, brain mass 5,145 g) to section for subsequent staining.

The diencephalon and brainstem (midbrain, pons and medulla oblongata) were dissected from the remainder of the brain as a single block of tissue. This block of tissue was placed in $30 \%$ sucrose in $0.1 \mathrm{M} \mathrm{PB}$ at $4{ }^{\circ} \mathrm{C}$ until it had equilibrated. The tissue was then frozen in crushed dry ice and mounted onto an aluminium stage that was attached to a freezing microtome modified to allow the sectioning of large tissue blocks. The entire tissue block was sectioned on the coronal plane, with a section thickness of $50 \mu \mathrm{m}$. A 1-in-20 series of sections was taken, and the first 9 sections were stained for Nissl substance, myelin, choline acetyltransferase (ChAT), tyrosine hydroxylase (TH), serotonin (5HT), orexin/ hypocretin $(\mathrm{Ox})$, parvalbumin $(\mathrm{PV})$, calbindin $(\mathrm{CB})$, and calretinin (CR). The remaining 11 sections were not stained and are kept in storage in the antifreeze solution at $-20^{\circ} \mathrm{C}$ (allowing for further work if needed). Sections used for the Nissl series were 
mounted on $1 \%$ gelatine-coated glass slides, allowed to dry for 1 week, cleared for $24 \mathrm{~h}$ in a solution of $1: 1$ chloroform and $100 \%$ alcohol and then stained with $1 \%$ cresyl violet. The sections used for the myelin series were stored in $5 \%$ formalin for 2 weeks at $4{ }^{\circ} \mathrm{C}$ before being mounted on $1.5 \%$ gelatine-coated slides, allowed to dry for 1 week and then stained using a modified silver staining technique [Gallyas, 1979].

The sections for free-floating immunohistochemical staining were treated for $30 \mathrm{~min}$ in an endogenous peroxidase inhibitor (49.2\% methanol: $49.2 \% 0.1 \mathrm{M} \mathrm{PB}: 1.6 \%$ of $30 \%$ hydrogen peroxide) followed by three 10 -min rinses in $0.1 \mathrm{M} \mathrm{PB}$. Sections were then preincubated for $2 \mathrm{~h}$, at room temperature, in blocking buffer [ $3 \%$ normal goat serum (NGS) for TH, 5HT, Ox, PV, CB and CR sections; $3 \%$ normal rabbit serum (NRS) for ChAT sections, $2 \%$ bovine serum albumin (BSA) and $0.25 \%$ Triton-X in $0.1 \mathrm{M}$ $\mathrm{PB}]$. Thereafter, sections were incubated in the primary antibody solution in blocking buffer for $48 \mathrm{~h}$ at $4^{\circ} \mathrm{C}$. An anti-choline acetyltransferase antibody (ChAT, AB144P; Millipore, raised in goat) at a dilution of 1:1,500 was used to reveal cholinergic neurons. An anti-tyrosine hydroxylase antibody (TH, AB151; Millipore, raised in rabbit) at a dilution of 1:7,500 revealed the catecholaminergic neurons. Serotonergic neurons (5HT) were revealed using an anti-serotonin antibody (AB938; Millipore, raised in rabbit) at a dilution of 1:10,000. Orexinergic neurons (OxA) were revealed using an anti-orexin-A antibody (AB3704; Millipore, raised in rabbit) at a dilution of 1:3,000. To reveal parvalbumin immunoreactivity (PV) we used anti-parvalbumin antibody (PV-28; SWANT, raised in rabbit) at a dilution of $1: 20,000$. To reveal calbindin immunoreactivity $(\mathrm{CB})$ we used anti-calbindin antibody (CB-300; SWANT, raised in rabbit) at a dilution of 1:10,000. To reveal calretinin immunoreactivity (CR) we used anti-calretinin antibody (7699/4; SWANT, raised in rabbit) at a dilution of 1:10,000.

The primary antibody incubation was followed by three 10 min rinses in $0.1 \mathrm{MPB}$ and the sections were then incubated in a secondary antibody solution (a 1:1,000 dilution of biotinylated anti-goat IgG, BA-5000; Vector Labs, for ChAT sections, or a 1:1,000 dilution of biotinylated anti-rabbit IgG, BA-1000; Vector Labs, for the remaining sections, in a blocking buffer containing 3\% NRS/ NGS and $2 \% \mathrm{BSA}$ in $0.1 \mathrm{M} \mathrm{PB}$ ) for $2 \mathrm{~h}$ at room temperature. This was followed by three 10 -min rinses in $0.1 \mathrm{M} \mathrm{PB}$, after which sections were incubated for $1 \mathrm{~h}$ in Avidin-Biotin solution (Vector Labs, 1:125 dilution in $0.1 \mathrm{M} \mathrm{PB}$ ), followed by three 10 -min rinses in $0.1 \mathrm{M}$ PB. Sections were then placed in a solution of $0.05 \%$ $3,3^{\prime}$-diaminobenzidine (DAB) in $0.1 \mathrm{M} \mathrm{PB}$ for $5 \mathrm{~min}$, followed by the addition of $3 \mu \mathrm{l}$ of $3 \%$ hydrogen peroxide to each $1 \mathrm{ml}$ of solution in which each section was immersed. Chromatic precipitation was visually monitored and verified under a low-power stereomicroscope. Staining continued until such time as the background stain was at a level that would assist reconstruction without obscuring the immunopositive structures. Precipitation was arrested by placing sections in $0.1 \mathrm{M} \mathrm{PB}$, followed by two more rinses in this solution. Sections were then mounted on $1 \%$ gelatine-coated glass slides, air dried for 1 week at room temperature, dehydrated in a graded series of alcohols, cleared in xylene and coverslipped with Depex. To ensure lack of non-specific staining of the immunohistochemical protocol, we ran tests on five sections where we omitted the primary antibody, and five sections where we omitted the secondary antibody. In both cases no staining was observed. The observed immunostaining patterns support the specificity of the antibodies for the antigens in the African elephant, as they were compatible with observations made in other species [e.g. Bhagwandin et al., 2008, 2011; Gravett et al., 2009, 2011; Paxinos et al., 2009; Dell et al., 2010, 2012; Kruger et al., 2010, 2012; Pieters et al., 2010]. It was not feasible to undertake Western blot control testing in the elephant materials.

Sections were examined under a low-power stereomicroscope and, using a camera lucida, the architectonic borders of the sections were traced following the Nissl-and myelin-stained sections. Sections containing the immunopositive neurons were matched to the drawings and the neurons were marked. All drawings were then scanned and redrawn using the Canvas 8 drawing program. The architectonic nomenclature employed in this study was adapted from Jones [2007] and an atlas of the rat brain by Paxinos et al. [2009]. The nomenclature used for the cholinergic system was adapted from Woolf [1991], Limacher et al. [2008], Bhagwandin et al. [2008], Gravett et al. [2009], Dell et al. [2010] and Pieters et al. [2010], the catecholaminergic from Dahlström and Fuxe [1964], Hökfelt et al. [1984], Smeets and González [2000], Limacher et al. [2008], Bhagwandin et al. [2008], Gravett et al. [2009], Dell et al. [2010] and Pieters et al. [2010], the serotonergic from Törk [1990], Limacher et al. [2008], Bhagwandin et al. [2008], Gravett et al. [2009], Dell et al. [2010] and Pieters et al. [2010], and the orexinergic from Kruger et al. [2010], Bhagwandin et al. [2011], Gravett et al. [2011] and Dell et al. [2012]. While we used the standard nomenclature for the catecholaminergic system in this paper, we realize that the neuronal groups we revealed with tyrosine hydroxylase immunohistochemistry may not directly correspond with these nuclei as has been described in previous studies by Dahlström and Fuxe [1964], Hökfelt et al. [1976], Meister et al. [1988], Kitahama et al. [1990, 1996] and Ruggiero et al. [1992]; however, given the striking similarity of the results of the tyrosine hydroxylase immunohistochemistry to that seen in other mammals, we feel this terminology is appropriate. Clearly further studies in elephants with a wider range of antibodies, such as those to phenylethanolamine-N-methyltransferase (PNMT), dopamine- $\beta$-hydroxylase (DBH) and aromatic L-amino acid decarboxylase (AADC) would be required to fully determine the implied homologies ascribed in this study [e.g. Weihe et al., 2006].

\section{Results}

\section{Diencephalon}

The general appearance and location of the diencephalon of the African elephant, herein referring to the dorsal thalamus, ventral thalamus, epithalamus and hypothalamus, resembles that seen in most other mammals [Jones, 2007; Paxinos et al., 2009]. Our description is based on a template drawn from Jones' [2007] seminal work on the thalamus, but where the architecture differs we relied on descriptions of other species for both nomenclature and clarity [i.e. while Jones, 2007, provides a comprehensive template of the mammalian diencephalon, it does not always cover all aspects of mammalian diencephalic ana- 
tomical variation and does not include the hypothalamus; thus, we used Paxinos et al., 2009, as the template for description of the hypothalamus].

\section{Dorsal Thalamus}

The nuclear mass that comprises the dorsal thalamus of the elephant was found in a position typical of mammals, and while it is has been reported to be large it does not appear to be large in relation to the overall size of the elephant brain. As noted by Abe [1952] the internal medullary lamina is quite indistinct in many places, making the global divisions of anterior, medial, intralaminar and ventral nuclear complexes not particularly straightforward. Despite this, the typical mammalian nuclear groups, in positions typical of that seen across mammals [Jones, 2007], were evident, and these include the: ventral nuclei, medial geniculate complex, lateral geniculate nucleus, lateral posterior-pulvinar complex, posterior nuclear group, intralaminar nuclei, medial nuclei, and anterior nuclei (online suppl. fig. A-L; for all online suppl. material, see www.karger.com/doi/10.1159/000352004). Each of these nuclear groups is further subdivided, sometimes into several distinct nuclei, but again, for the most part, the individual nuclei within these groups conform to what has been observed in other mammals [Jones, 2007]. A more comprehensive description of each of the nuclear groups follows.

\section{The Ventral Nuclei}

The Ventral Posterior Nuclei. The ventral posterior nuclei represent the thalamic nuclei where the ascending trigeminal and medial lemniscal $(\mathrm{ml})$ pathways terminate in the dorsal thalamus. This nuclear cluster exhibited an ovoid shape, occupying almost the entire ventral aspect of the mid-level dorsal thalamus (online suppl. fig. E-J). Four distinct nuclei were observed in the ventral posterior nuclear complex in the elephant, these being the ventral posterior medial (VPM), ventral posterior lateral (VPL), ventral posterior inferior (VPI) and basal ventromedial $(\mathrm{Vmb})$ nuclei. The architecture of the VPM and VPL was very similar, but these nuclei could be distinguished on the basis of the presence of a thin, heavily myelinated, arcuate lamella separating the two nuclei. In the elephant, the VPL nucleus was slightly larger than the VPM nucleus, but not substantially so. In both nuclei the larger, intensely stained (for Nissl substance), relay cells exhibited a lobulated appearance due to the investment of afferent fibres, but this was more marked in the VPL than in the VPM where the distribution of the relay cells was more homogeneous. Despite this difference in the extent of the lobular appearance, no distinct lamella that could be directly related to different body parts of the elephant, as seen in mammals such as primates and rodents [Jones, 2007], was apparent. Within both nuclei a highdensity, homogenous distribution of interneurons immunoreactive for calbindin and a lower-density, homogenous distribution of interneurons immunoreactive for calretinin were observed. Both nuclei exhibited a moderately densely stained neuropil immunoreactive for parvalbumin.

A distinct VPI nucleus was found at the very ventral aspect of the ventral nuclear complex, occupying a position ventral to the arcuate lamella and dorsal to the zona incerta. Within this nucleus the density of larger relay cells was lower than that seen in the VPM and VPL, and while it has been argued that this is the result of the entry of the medial lemniscus into the VPL [Jones, 2007], the density of cells immunoreactive for both calbindin and calretinin was similar to that seen in the VPM and VPL. Medial and ventral to the VPM nucleus the Vmb nucleus was observed. This nucleus was readily distinguished from the VPM due to the lack of large relay cells and the presence of densely packed small to medium sized neurons. As with the other nuclei of the ventral posterior group, a high density of calbindin and a lower density of calretinin-immunoreactive neurons, and a neuropil immunoreactive for parvalbumin, were observed. The presence of the $\mathrm{Vmb}$ nucleus belies the observation of the lack of taste receptors in the tongue of the elephant [Kubota, 1966], and it is likely that observations on better quality material of the elephant tongue will reveal taste receptors in the circumvallate papillae.

The Ventral Lateral Nuclei. The ventral lateral nuclei of the elephant occupy the ventral nuclear mass rostral to the ventral posterior nuclei (online suppl. fig. A-D). This mass extends through to the rostral pole of the dorsal thalamic nuclear mass and was divisible into 3 nuclei in the elephant: a ventral anterior nucleus (VA), a ventral lateral nucleus (VL) and a smaller, principal ventral medial nucleus (VMP). In all three nuclei, large, intensely stained (for Nissl substance) relay cells were evident in a low to moderate density. We could not find any evidence for anterior and posterior divisions of the VL or a parvocellular division of VA as observed in primates [Jones, 2007]. Throughout all three nuclei a high density of calbindin and a lower density of calretinin-immunoreactive neurons, and a neuropil immunoreactive for parvalbumin, were observed. While the outer borders of the ventral lateral nuclei were readily defined, the borders of the nuclei when juxtaposed within the complex were not so clear. A 
distinguishing feature of the VL nucleus was the distinct lobulated appearance of the relay cells, with large intervening fascicules. The VA nucleus, which was found dorsal and rostral to the VL, did not exhibit such distinct lobulation of the relay cells. The VMP nucleus, which was located on the ventromedial tip of the VL, did not exhibit any lobulation, with the relay cells exhibiting a homogeneous distribution throughout the nucleus. While we were able to distinguish a border between the VMP and the VA, this border may represent a transitional zone between the two nuclei, as it has been proposed that the VA is a rostral extension of the VMP [Jones, 2007]. In smaller mammals, the VA nucleus is difficult to identify, but in primates both the VMP and the VA are in receipt of nigral inputs [Jones, 2007]. Given the large size and unusual appearance of the elephant substantia nigra (see below), it is possible that the elephant may show a distinct VA nucleus not recorded in other mammals. Even if the VA nucleus was just a rostral extension of the VMP nucleus, the large size of the region designated as VA in the elephant is likely related to the large substantia nigra. It is possible that with connectional studies and other stains more nuclei would be evident in the elephant; however, the overall appearance of the ventral lateral nuclei in the elephant is reminiscent of that seen in cats [Jones, 2007].

\section{The Medial Geniculate Complex}

The medial geniculate nuclear complex was the most caudal nuclear mass observed in the dorsal thalamus of the elephant, forming a distinct, large, caudolateral bulge on the surface of the diencephalon extending caudal to the optic tract (online suppl. fig. K, L). Within the medial geniculate complex we found the three classically identified mammalian nuclei, the ventral (MGv), dorsal (MGd) and medial $(\mathrm{MGm})$ medial geniculate nuclei [Jones, 2007], as well as a distinct marginal zone of the medial geniculate (MzMg) [Paxinos et al., 2009] and the transverse infrageniculate nucleus (TIN) described by Precechtel [1925] and Abe [1952]. The largest of the medial geniculate nuclei was the MGd, which occupied the upper half of the medial geniculate complex throughout its full rostrocaudal extent. The larger relay cells found within the MGd appeared to be organized in rough dorsoventrally oriented columns throughout the nucleus. The MGd was readily distinguished from the other medial geniculate nuclei by the presence of a homogeneous low density of parvalbumin-immunoreactive neurons, and a high density of calretinin-immunopositive neurons. Calbindin-immunopositive neurons were found in a high homogeneous density throughout the MGd. Ventral to the MGd, the MGv nucleus was evident through the rostrocaudal extent of the medial geniculate complex. Within the MGv the relay cells appeared to be oriented in roughly horizontal columns, making this nucleus readily distinguishable from the MGd. In addition, the MGv exhibited a lower density of calretinin-immunopositive neurons and, while having a neuropil immunoreactive for parvalbumin, displayed no parvalbumin-immunopositive cells. The MGv was slightly smaller in size than the MGd. At the medial aspect of the MGv, between it and the brachium of the inferior colliculus, a distinct region of slightly larger relay cells was assigned to the MGm nucleus. This nucleus, apart from cell size, was distinguished from the MGv on the basis of the larger relay cells being arranged in more distinct columns that were oriented on a dorsomedial to ventrolateral plane. Staining for parvalbumin, calbindin, and calretinin in the MGm was the same as that seen for the MGv. The MGm was relatively small in size when compared to the MGd and MGv nuclei.

Small, but distinct, clusters of neurons surrounding the caudal edges of the MGd, MGv and MGm formed what is termed here the marginal zone of the medial geniculate (MzMG). The cells within the MzMG were clearly not thalamic relay cells due to their small size and appeared to have a more flattened, almost fusiform morphology, which is not typical of thalamic relay cells. These clusters lacked any immunoreactivity for parvalbumin and calbindin but appeared to contain a small number of calretinin-immunopositive neurons. The transverse infrageniculate nucleus (TIN) appears to be a novel nucleus of the medial geniculate complex only found in elephants (fig. 2). This moderately large nucleus was observed to share a medial border with the ventral and lateral aspects of the MGv and a lateral border with the optic tract, making it the most ventral and lateral nucleus of the medial geniculate complex. Interestingly, this nucleus was relatively cell sparse, with thalamic relay neurons only being found in the ventrolateral half of the nucleus. In the mediodorsal half of the nucleus a small number of calretininimmunopositive neurons were found. This segregation of relay cells and inhibitory interneurons gives the TIN a bilaminate appearance. The TIN was readily distinguished from the MGv not only by these two features but also by the lower density of calbindin-immunopositive neurons within the nucleus. It is difficult to determine on the basis of the current study whether the TIN is a completely novel elephantine nucleus, or whether it is a specialized portion of the MGv. 


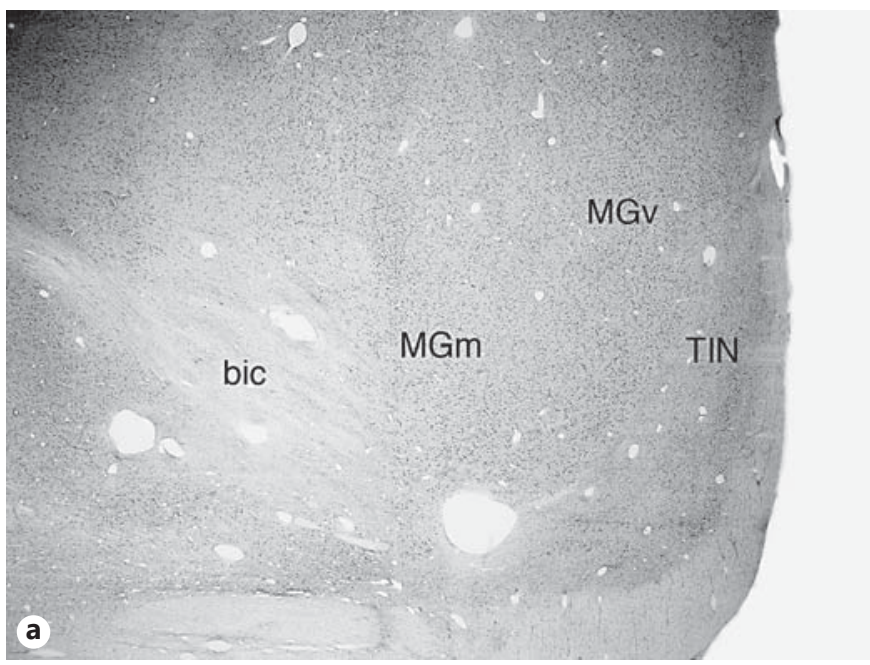

Fig. 2. Low-power photomicrographs of the ventral half of the medial geniculate complex demonstrating the location of the transverse infrageniculate nucleus (TIN), a novel medial geniculate nucleus in the elephant dorsal thalamus, in adjacent sections. a Nissl stain. b Myelin stain. c Calretinin stain. Medial is to the left and dorsal to the top. Scale bar in $\mathbf{b}=5 \mathrm{~mm}$ and applies to all. bic $=$ Brachium of the inferior colliculus; $\mathrm{MGm}=$ medial geniculate nucleus, medial division; $\mathrm{MGv}=$ medial geniculate nucleus, ventral division.

The Lateral Geniculate Nucleus

As pointed out by Jones [2007] the lateral geniculate nucleus, as referred to across mammals, should really be considered a nuclear complex, as it is composed of more than one nucleus. In the elephant we readily recognized a dorsal lateral geniculate nucleus (LGd), a ventral lateral geniculate nucleus (LGv) and an intergeniculate leaflet (IGL) (the latter two are described below as they form part of the ventral thalamus). In the elephant this nuclear complex was observed on the dorsolateral surface of the dorsal thalamic nuclear mass and was completely segregated from the main nuclear mass by a thin sheet of white matter (online suppl. fig. I, J). The dorsal lateral geniculate nucleus has a variable appearance across mammals, but the appearance of this nucleus in the elephant resembles mostly what is described as the 'rodent'
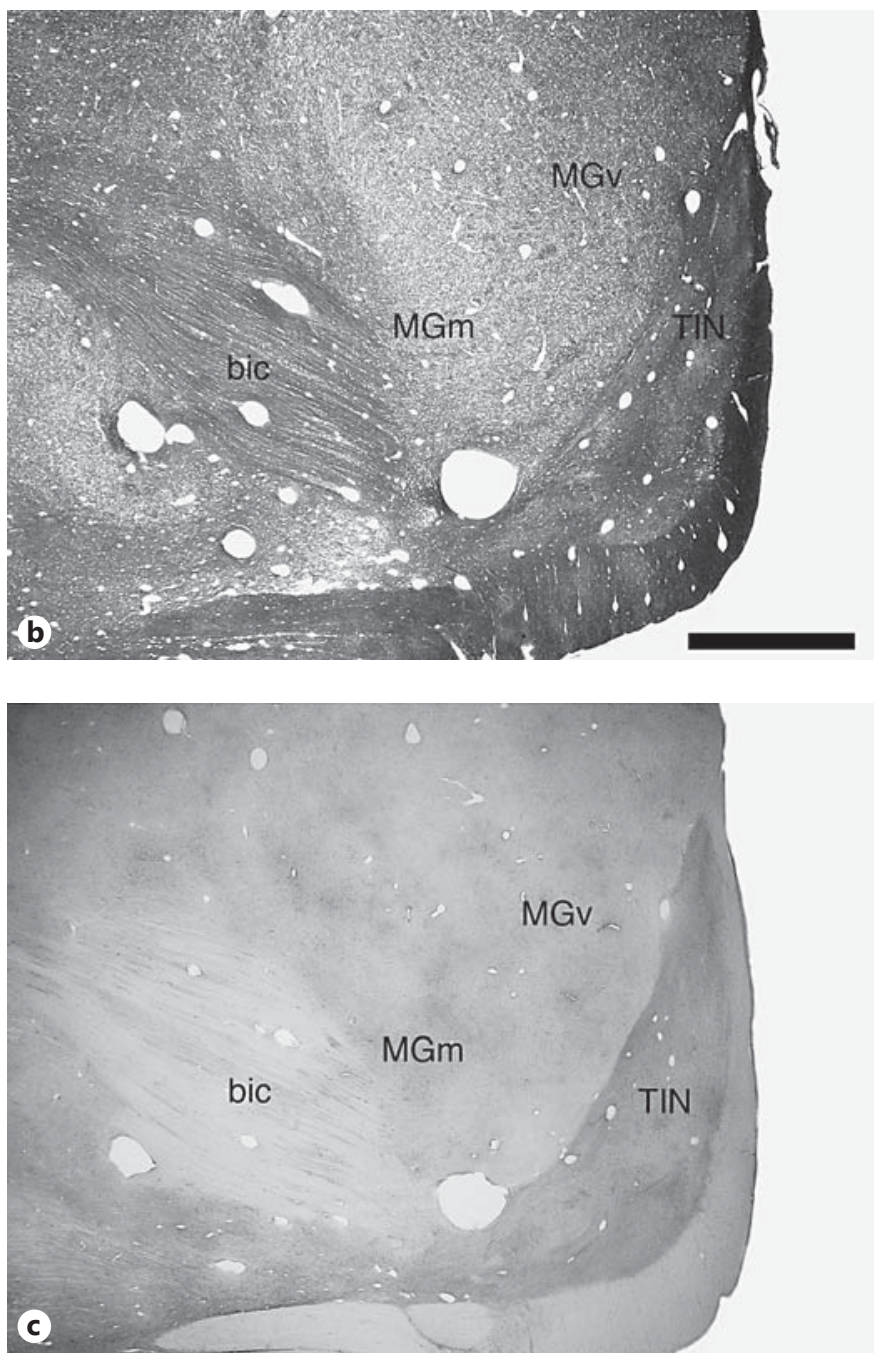

or 'generalized' form of the nucleus by Jones [2007]. The main mass of the dorsal lateral geniculate nucleus in the elephant was composed of a homogeneous mass of neurons of various sizes, with many of the smaller neurons immunopositive for calbindin and calretinin. We could not identify any lamination on the basis of the stains used in the current study, or a region that could be identified as part of the dorsal lateral geniculate nucleus that received input from the ipsilateral eye; however, tracing studies from the retina may reveal either lamination or an ipsilateral region, but to undertake such a study, while interesting, would be a very difficult task. On the very dorsal aspect of the dorsal lateral geniculate nucleus we observed a thin lamina of cells that evinced an intense immunoreactivity to parvalbumin in the neuropil (fig. 3). We have referred to this lamina as the cap of the lateral 

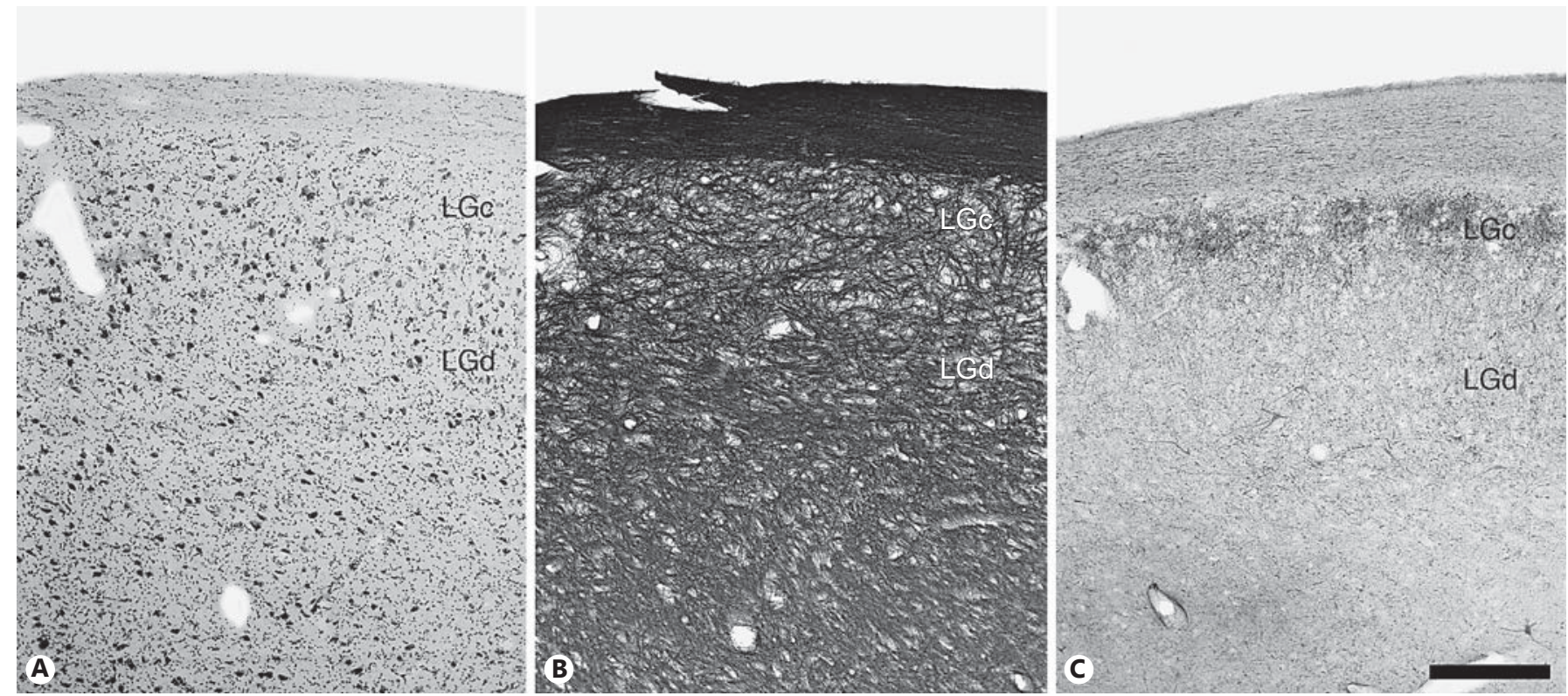

Fig. 3. Low-power photomicrographs illustrating the 'cap' region of the lateral geniculate nucleus (LGc) that lies above the dorsal lateral geniculate nucleus (LGd). a Nissl stain. b Myelin stain. c Parvalbumin stain. Note the dense parvalbumin-immunopositive neuropil within the LGc. Medial is to the left and dorsal to the top. Scale bar in $\mathbf{c}=500 \mu \mathrm{m}$ and applies to all.

geniculate nucleus (LGc), and it likely corresponds to the small-celled region in rodents [or intercalated lamina in primates, Jones, 2007] in receipt of tectal afferents. Our observations indicate that the lateral geniculate nuclear complex of the elephant can be considered to be a very typical or generalized form of lateral geniculate nucleus as described by Jones [2007].

\section{The Lateral Posterior-Pulvinar Complex}

A large block of the dorsal thalamic nuclear mass, located in the dorsolateral mid-level of the elephant dorsal thalamus, is considered here as the lateral posterior (LP)pulvinar complex (online suppl. fig. C-K) based on the recommendation with regard to the terminology of this region by Jones [2007]. In his cross-species comparison of this region of the dorsal thalamus, Jones [2007] identified five specific 'anchoring regions' that appear to demarcate this complex across mammalian species. Jones indicates that rostrally the LP-pulvinar complex should be adjacent to the posterior border of the ventral lateral nucleus. In the elephant, the rostral border of the LP-pulvinar complex is found dorsal to the posterior border of the ventral anterior nucleus and separated from this nucleus by a distinct myelin-dense lamella. Medially, the elephant LP-pulvinar complex is separated from both the superior colliculus and the pretectal area by a thin myelinated lamella, close to the suprageniculate nucleus, which is similar to the second anchor described by Jones [2007]. Laterally, the elephant LP-pulvinar complex is also separated from the lateral geniculate nucleus by a thin myelinated lamella, forming the third anchor of this region as seen in other mammals. The fourth anchor region present in the elephant LP-pulvinar complex is the ventral border of the LP-pulvinar complex being in apposition with the medial nucleus of the posterior nuclear complex. Lastly, Jones [2007] described the fifth anchor region being where the posterior aspect of the LP-pulvinar complex fades into the dorsal part of the medial geniculate complex. In the elephant the LP-pulvinar complex is clearly segregated from the medial geniculate complex by a distinct heavily myelinated lamella. Thus, the elephant LP-pulvinar complex clearly exhibits three of the five anchors as described by Jones [2007], while the remaining two anchors are in very similar positions, but with intervening myelinated lamella. Given this, we can be confident that the region outlined in the current study as the LP-pulvinar complex of the elephant is indeed this nuclear mass.

Across mammalian species, the LP-pulvinar complex is known to have several distinct subdivisions, but these 
are perhaps the most contentious architectural subdivisions of the dorsal thalamus, with several different authors outlining several different schemata, even in the same species [Jones, 2007]. In the elephant we did not see any distinct and reliable enough demarcations that could be used to subdivide the LP-pulvinar complex. From the lateral aspect of the LP-pulvinar complex a number of large fascicules enter the LP-pulvinar complex and give the lateral half to two thirds of this nuclear mass a striated appearance. One may elect to use the termination of the investing fasciculi as a marker to subdivide the LP-pulvinar complex into medial and lateral nuclei, but the homogeneous nature of the relay cell population, a homogenous high density of calbindin- and calretinin-immunoreactive neurons, and a homogenous neuropil immunoreactivity for parvalbumin argue against this parcellation. It is highly likely that connections, such as projection from the superior colliculus, or histochemistry for acetylcholine, would provide accurate parcellation of the elephant LP-pulvinar complex into several distinct nuclei, but given the absence of this data it is most prudent to currently consider this a singular nuclear mass.

\section{The Posterior Group of Nuclei}

The posterior group of nuclei appears to be a historical construct composed of nuclei that can, using modern techniques, be assigned to other nuclear complexes, such as the intralaminar nuclei, the lateral posterior-pulvinar complex or the medial geniculate complex [Jones, 2007]. Despite these possibilities, the posterior group of nuclei has not yet undergone such a transformation, as further information is required in order to make these various assignations and re-establish the placement of these nuclei; thus, for the purposes of the current description, we maintain the posterior group of nuclei as a separate entity within the dorsal thalamic nuclear mass. Normally, the posterior group of nuclei is thought to contain a limitans-suprageniculate nucleus and a posterior nucleus (made up of medial, lateral and intermediate divisions) [Jones, 2007]. In the African elephant we could readily identify a distinct limitans nucleus, as well as dorsal and ventral subdivisions of the suprageniculate nucleus (online suppl. fig. E-K). The posterior nucleus was observed to be quite large in the elephant, and while it likely contained the subdivisions normally recognized in carnivores and primates, these were not readily identifiable on the basis of the stains used in the current study.

The limitans and suprageniculate nuclei were located in the caudal, medial aspect of the dorsal thalamic nuclear mass, medial to the medial geniculate complex, caudal to the ventral posterior nuclei, lateral to the pretectal region, and medioventral to the lateral posterior-pulvinar complex and the brachium of the inferior colliculus. The limitans nucleus was most readily observable when it was located ventral and medial to the brachium of the inferior colliculus. In this location a moderate-density, wide band (3-4 mm) of deeply stained (for Nissl substance) relay cells was evident. The limitans nucleus was also readily delimited due to the presence of a parvalbumin-immunopositive neuropil (not present in the suprageniculate nuclei), a high density of calbindin-immunoreactive neurons (not present in the ventral suprageniculate nucleus) and a moderate density of calretinin-immunopositive neurons (whereas there is a high density of these neurons in the dorsal suprageniculate nucleus). The rostral portion of the limitans nucleus was observed to be isolated as small clusters of cells invested in the posterior nucleus and the lateral posterior-pulvinar complex.

Dorsomedial and ventromedial to the limitans nucleus were the dorsal suprageniculate and ventral suprageniculate nuclei, respectively. The dorsal suprageniculate nucleus was located in the dorsal medial aspect of the caudal portion of the dorsal thalamic nuclear mass, sharing a medial border with the superior colliculus/pretectal area, a dorsolateral border with the lateral posterior-pulvinar complex, a ventral border with the ventral suprageniculate nucleus and a lateral border with the limitans nucleus and the brachium of the inferior colliculus. The density of relay cells in the dorsal suprageniculate nucleus was moderate, and they were all intensely stained (for Nissl substance). This nucleus lacked a parvalbumin-immunopositive neuropil and had high densities of both calbindin- and calretinin-immunopositive neurons. These features made this nucleus readily distinguishable from those in the adjacent dorsal thalamic nuclear mass. The ventral suprageniculate nucleus occupied the ventromedial corner of the caudal portion of the dorsal thalamic nuclear mass and shared a dorsal medial border with the dorsal suprageniculate nucleus and the limitans nucleus in the caudal aspect and the posterior nucleus and the ventral posterior nuclei in its more rostral aspect. In the ventral suprageniculate nucleus the density of palely stained (for Nissl substance) relay cells was significantly lower than in the surrounding nuclei, and this nucleus lacked a parvalbumin-immunopositive neuropil and had a high density of calbindin-immunopositive neurons and a low to moderate density of calretinin-immunopositive neurons. The neurons forming this nucleus were seen to extend laterally, ventral to the limitans nucleus, to almost reach the medial geniculate complex. The general appear- 
ance and location of these three nuclei, the limitans and suprageniculate nuclei, in the elephant are consistent with those observed in other mammals [Jones, 2007].

In the elephant the posterior nucleus was quite large, being located, for the most part, ventral to the lateral posterior-pulvinar complex, dorsal to the ventral posterior nuclei and, in the more rostral aspects, lateral to the intralaminar nuclei. The appearance of cells throughout the region designated as the posterior nucleus in the elephant was homogenous, with a low to moderate density of palely staining (for Nissl substance) relay cells being found throughout the nucleus. This nucleus also exhibited a parvalbumin-immunopositive neuropil and had a high density of calbindin-immunopositive neurons and a low to moderate density of calretinin-immunopositive neurons throughout. This combination of features made the delineation of the borders of this nucleus reasonably straightforward. Jones [2007] has argued that the portion of the posterior nucleus overlying the ventral posterior nuclei should be designated as the medial subdivision of the posterior nucleus, and this would be consistent for the elephant. The lateral subdivision of the posterior nucleus, indicated by Jones [2007] to lie in a position lateral to the ventral posterior nuclei, does not appear to be evident in the elephant, nor does the intermediate division of the posterior nucleus, which Jones [2007] indicates to be an expansion of the posterior nucleus located beneath the lateral posterior-pulvinar complex extending towards the medial geniculate complex. Thus, in the elephant, in comparison to other mammals, the posterior nucleus appears to be composed mostly of what would be called the medial division of the posterior nucleus in other mammals [Jones, 2007]. A significant rostromedial expansion of the posterior nucleus in the elephant is evident (online suppl. fig. E-K), lying between the rostromedial poles of the ventral posterior nuclei and the lateral posterior-pulvinar complex. While this may still form part of the medial division of the posterior nucleus, this expansion may be more related to the lateral posterior-pulvinar complex [like the anterior pulvinar nucleus of primates, Jones, 2007] than the ventral posterior nuclei. Potentially, further examination with a greater range of stains than used in the current study may determine whether the entire posterior nucleus of the elephant should be considered a direct homology of the medial division of the posterior nucleus of other mammals, or whether additional subdivisions are evident.

\section{The Intralaminar Nuclei}

The intralaminar nuclei are those generally thought of as being within or associated with the internal medullary lamina; however, the internal medullary laminar is not a distinct structure in the elephant, as seen in other mammals, making the delineation of these nuclei in the elephant a more difficult task. Moreover, the nuclei associated with the midline of the dorsal thalamic nuclear mass have been included in the intralaminar nuclei [Jones, 2007]. The intralaminar nuclei are generally divided into a rostral group (containing the central medial, paracentral, central lateral and rhomboid nuclei) and a caudal group (containing the centre médian and parafascicular nuclei) [Jones, 2007], although this terminology appears confusing, as the centre médian nucleus, forming part of the posterior group of intralaminar nuclei, is clearly located in a position rostral to the central medial nucleus, part of the anterior group, in the elephant and in many other mammalian species [Jones, 2007]. Moreover, in the elephant, the parafascicular nucleus and the centre médian nucleus do not appear to share any topological similarity, making their grouping appear to be of no purpose. Given these difficulties, in the current description of the intralaminar nuclei in the elephant we will group all of the nuclei assigned to the intralaminar nuclei under the one rubric and describe them in a rostral to caudal progression to attempt to prevent any interpretational difficulties (online suppl. fig. B-I).

The most rostral intralaminar nuclei encountered are found approximately $10 \mathrm{~mm}$ from the rostral pole of the dorsal thalamic nuclear mass and include the rhomboid, centre médian and central lateral nuclei. The rhomboid nucleus in the elephant is located immediately ventral to the third ventricle, spanning across the midline (in the massa intermedia thalami) as a single, clearly rhomboidshaped, nuclear mass. This nucleus is, in comparative terms, not particularly large (with a maximum rostrocaudal extent of less than $5 \mathrm{~mm}$ ). While it had a high density of intensely stained (for Nissl substance) neurons and a high density of calbindin-immunopositive neurons, no other structures were stained in this nucleus with the techniques used in the current study. In a position immediately caudal to and lateral to the rhomboid nucleus, and dorsal to the medioventral nucleus (see below), a distinct centre médian nucleus was observed. This nucleus also spanned the midline as a single nuclear mass and had small wing-like extensions from the lateral aspect of the nucleus extending towards the central lateral nucleus. The centre médian nucleus was substantially larger than either the rhomboid or the medioventral nuclei, with a rostrocaudal extent of over $15 \mathrm{~mm}$. It was readily distinguished from the surrounding nuclei by having horizontally oriented columns of cells that extended into the 
wings of this nucleus. Moreover, the centre médian nucleus had a neuropil immunoreactive for parvalbumin and high densities of both calbindin- and calretinin-immunopositive neurons.

A distinct central lateral nucleus was observed to lie between the medial nuclei (medial border) and the lateral posterior-pulvinar complex and posterior nucleus (lateral border), within the incipient internal medullary lamina. This nucleus was composed of a high density of intensely stained (for Nissl substance) neurons arranged in rows running parallel to the curved borders of this nucleus. This wing-like extension of the midline nuclei was observed to curve around the lateral aspect of the medial nuclei and was found in this location until the caudal level coincident with the fasciculus retroflexus. The medial border of this nucleus was readily distinguished from the centre médian nucleus by the absence of any parvalbumin-immunopositive neuropil, although as with the centre médian nucleus a high density of calbindin- and calretinin-immunopositive neurons was found homogenously distributed throughout the nucleus. Caudal to the centre médian nucleus, a large nuclear mass, the central medial nucleus, was observed. The central medial nucleus was, as in other mammals, the largest of the intralaminar nuclei in the elephant, with a mediolateral extent of over $15 \mathrm{~mm}$, a dorsoventral extent of over $5 \mathrm{~mm}$, and a rostrocaudal extent of approximately $15 \mathrm{~mm}$, and was seen to lie in the incipient internal medullary lamina. This nucleus was readily distinguished from the surrounding nuclei by the incipient internal medullary lamina, and also by the low to moderate density of slightly smaller relay cells homogeneously distributed throughout. While there was a high density of calbindin-immunopositive neurons throughout the nucleus, no calretinin-immunoreactive neurons or parvalbumin-immunopositive neuropil were observed in this nucleus, which were also useful markers of the limits of this nucleus. The last intralaminar nucleus that we could clearly distinguish in the elephant was the parafascicular nucleus. Unlike in other mammals, this nucleus was only found laterally to the fasciculus retroflexus, and not pierced by this fibre bundle. The parafascicular nucleus was found in a position dorsal to the central medial nucleus and caudal to the medial nuclei, at first appearing as though it were an extension of the central lateral nucleus. It was readily distinguished from the central lateral nucleus by a thin fibrous lamella, a higher density of neurons and a parvalbumin-immunopositive neuropil.

\section{The Medial Nuclei}

The medial nuclear complex is composed of the medial dorsal nucleus, the parataenial nucleus, and the medioventral or reuniens nucleus based on the developmental profile of this thalamic region [Jones, 2007]. In the elephant we observed a medial dorsal nucleus that had three clear subdivisions (dorsal, medial and ventral), a distinct parataenial nucleus, a distinct medioventral nucleus, reuniens nuclei (with the reuniens nucleus having a distinct ventral subdivision), and a distinct intermediodorsal nucleus (online suppl. fig. A-G). Thus, the medial nuclear complex in the elephant is clearly more parcellated than that seen in many other mammals. The majority of the medial nuclear complex was occupied by a large medial dorsal nucleus that exceeds a cross-sectional diameter of $15 \mathrm{~mm}$ at its largest and has a rostrocaudal extent of over $20 \mathrm{~mm}$. The medial dorsal nucleus, on the basis of the stains used in the current study, could be readily divided into three distinct subdivisions. The largest of these three subdivisions was the dorsal subdivision, which occupied the lateral and dorsal aspects of the nucleus throughout its extent. Within this subdivision of the medial dorsal nucleus, the relay cells were observed in a moderate density and were slightly more palely stained (for Nissl substance) than the surrounding nuclei. In addition, this medial subdivision lacked any parvalbuminimmunopositive neuropil but contained high densities of calbindin- and calretinin-immunopositive neurons. Interestingly, within this medial subdivision, we noted large, multipolar calbindin-immunopositive neurons that exhibited extensive dendritic arbors (fig. 4a). This neuronal type was not observed in the medial subdivision but was evident in the ventral subdivision. For the most part, the architecture of the ventral subdivision of the medial dorsal nucleus was similar to that seen in the dorsal subdivision, but parvalbumin-immunopositive neurons were readily identified within the ventral subdivision, whereas these were absent in the dorsal subdivision. The ventral subdivision was found to border the medioventral border of the rostral portion of the dorsal subdivision. Adjacent to the dorsomedial aspect of the dorsal subdivision, a distinct medial subdivision of the medial dorsal nucleus was evident throughout the medial nuclear complex. This medial subdivision contained slightly larger neurons packed at a higher density than observed in the dorsal and ventral subdivisions [perhaps equating it with the magnocellular subdivision of this nucleus in primates, Jones, 2007]. In addition, this medial subdivision contained parvalbumin-immunopositive neurons (fig. $4 \mathrm{~b}$ ) and, while also housing a high density of calbindin- and 


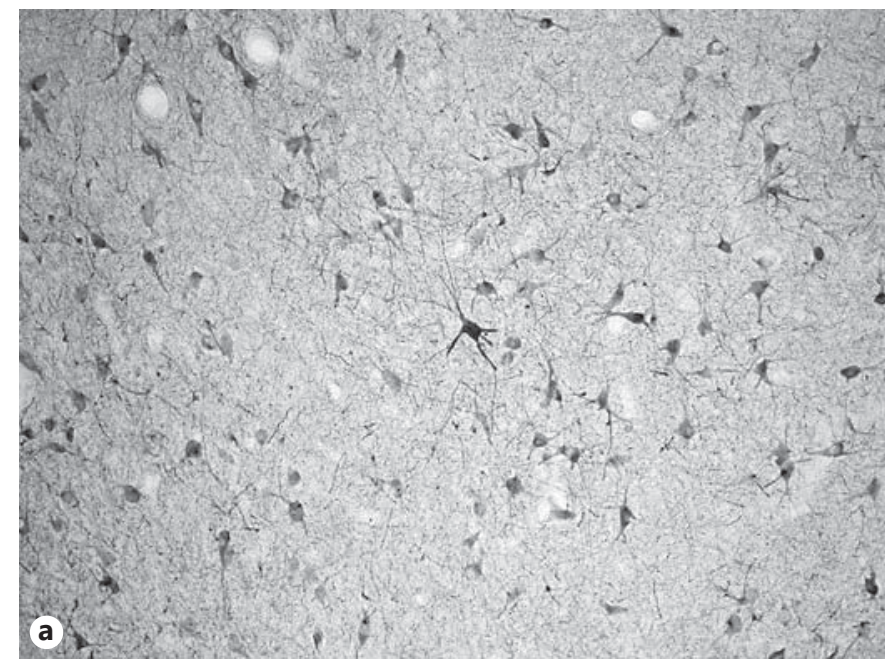

Fig. 4. Photomicrographs of calbindin- (a) and parvalbumin-immunopositive neurons (b) in the medial division of the dorsomedial thalamic nucleus (MDd). Note that while most of the calbindin-immunopositive neurons are moderately intensely stained and do not exhibit extensive dendritic arbors, the occasional cal-

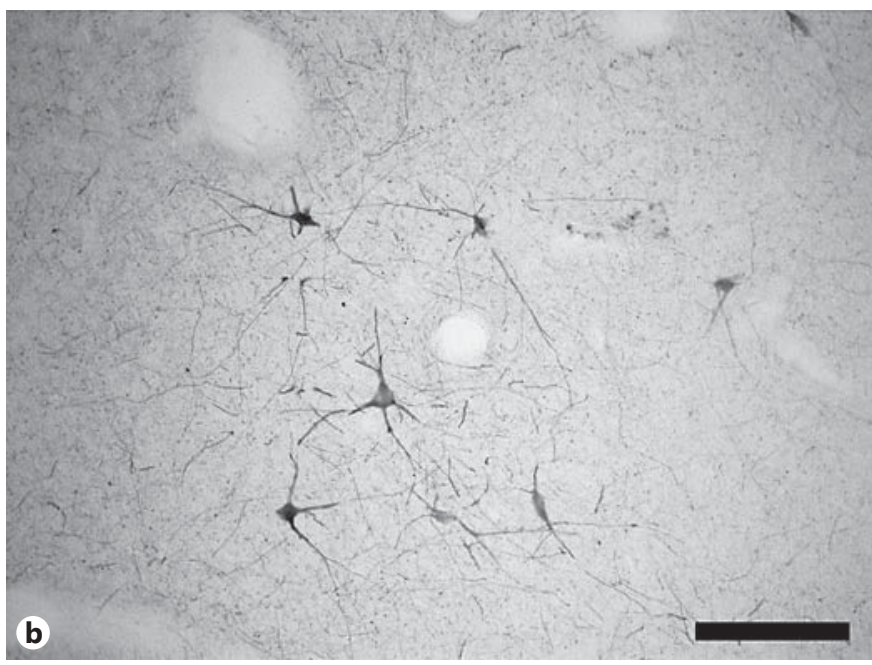

bindin-immunopositive neuron in this region of the dorsal thalamus was more intensely stained and exhibited extensive dendritic arborization. Medial is to the left and dorsal to the top. Scale bar in $\mathbf{b}=250 \mu \mathrm{m}$ and applies to both. calretinin-immunopositive neurons, did not contain any of the larger calbindin-immunopositive neurons observed in the dorsal and ventral subdivisions of this nucleus. Rostral and medial to the medial dorsal nucleus, just ventral to the stria medullaris thalami, a small band of neurons at the most rostromedial margin of the dorsal thalamic nuclear mass, found between the anteromedial nucleus (see below) and the paraventricular nucleus (see below), was assigned to the parataenial nucleus. This nucleus shows an architectural appearance very similar to that of the dorsal division of the medial dorsal nucleus, including the presence of a low density of larger calbindin-immunopositive neurons, and this feature allowed the distinction of this nucleus from the medial division of the medial dorsal nucleus.

Immediately ventral to the rhomboid nucleus (see above) in the midline grey matter was a distinct region of lower cell density that was assigned to the medioventral nucleus. This nucleus also spanned the midline as a single nuclear mass and was bordered inferiorly by the third ventricle and laterally by the ventral nuclear complex. The pattern of immunostaining observed in this nucleus was similar to that seen in the rhomboid nucleus; thus, the feature defining the separation of these two nuclei was the lower cell density in the medioventral nucleus. The medioventral nucleus was slightly larger than the rhomboid nucleus and extended a further $5 \mathrm{~mm}$ caudal to the caudal pole of the rhomboid nucleus. Approximately $5 \mathrm{~mm}$ caudal to the caudal pole of the medioventral nucleus, a distinct reuniens nucleus, with a clear ventral reuniens wing extending from the lateral aspect of the nucleus, was evident in a position ventral to the caudal-most aspect of the centre médian nucleus. At this level, the reuniens nucleus was U-shaped in appearance and was distinguished from the centre médian nucleus by exhibiting a higher density of slightly larger, more deeply stained (for Nissl substance) neurons than the centre médian nucleus. This nucleus exhibited no parvalbumin immunoreactivity but contained high densities of calbindin- and calretinin-immunopositive neurons. No specific architectural differences were noted between the ventral reuniens nucleus and the reuniens nucleus but, due to the location of this nucleus as a clear lateroventral extension of the main nuclear mass, this distinction is provided, although it should be considered a tenuous distinction. At a similar rostrocaudal level, a distinct intermediodorsal nucleus was observed between the centre médian nucleus (see above) and the wall of the third ventricle forming the dorsal limit of the mass intermedia thalami. The intermediodorsal nucleus formed a nuclear mass that encircled the third ventricle, spanning the midline as a single nuclear mass. While there were no major features that distinguished this nucleus from the centre médian nucleus, the lack of parvalbumin-immunopositive neuropil pro- 
vided a distinct architectural border. At an approximately similar level of the dorsal thalamus, distinct reuniens and ventral reuniens nuclei were observed.

\section{The Anterior Nuclei and Lateral Dorsal Nucleus}

Three anterior nuclei are typically observed in mammals: the anterodorsal, anteroventral and anteromedial nuclei [Jones, 2007]. Based on topological and connectional grounds, Jones [2007] also includes the lateral dorsal nucleus in this nuclear complex, and we follow this designation herein. In the elephant all four of these nuclei, in their typically mammalian positions, were readily observed, but the distinction of this complex from the remainder of the dorsal thalamic nuclear mass based on the rostral bifurcation of the internal medullary lamina was not distinct due to the incipient nature of this lamina in the elephant (online suppl. fig. A-F). Of the three anterior nuclei observed in the elephant, the anteromedial nucleus was by far the largest (about twice the size of the other nuclei combined), with the anteroventral and anterodorsal nuclei being of a similar size. The anteromedial nucleus was located dorsomedially to the medial dorsal nucleus, i.e. between it and the stria medullaris thalami. A moderate density of relay cells was distributed homogeneously throughout the nucleus, and while it possessed the typical high density of calbindin- and calretinin-immunoreactive neurons, this nucleus also exhibited a low density of parvalbumin-immunoreactive neurons. Located between the dorsolateral border of the anteromedial nucleus and the medial border of the lateral dorsal nucleus (see below), a small nuclear mass divisible into the anteroventral and anterodorsal nuclei was located. The anteroventral nucleus exhibited an architecture that was very similar to that of the anteromedial nucleus, but this nucleus lacked the parvalbuminimmunopositive neurons observed in the anteromedial nucleus. In addition, larger, extensively arborized, calbindin-immunopositive neurons were observed in the anteroventral nucleus, whereas these were not seen in the anteromedial nucleus. The anterodorsal nucleus was observed to lie immediately dorsal to the anteroventral nucleus. The anterodorsal nucleus was distinguished from the anteroventral nucleus by the greater density of relay cells throughout the nucleus, although this is not as marked a difference as seen in mammals with smaller brains [Jones, 2007]. This nucleus also housed the larger, extensively arborized, calbindin-immunopositive neurons observed in the anteroventral nucleus and lacked the parvalbumin-immunopositive neurons observed in the anteromedial nucleus. Thus, the stains used in the current study allowed us to readily delineate three anterior nuclei in the elephant.

The lateral dorsal nucleus formed a distinctive 'cap' over the dorsal surface of the dorsal thalamic nuclear mass, having a mediolateral dimension of up to $15 \mathrm{~mm}$ and a rostrocaudal dimension of approximately $20 \mathrm{~mm}$, with a dorsoventral extent of 3-5 mm. This nucleus extends from the rostral portion of the dorsal thalamus, overlying the rostral two thirds of the lateral posteriorpulvinar complex. While being invested with high densities of calbindin- and calretinin-immunopositive neurons, this nucleus lacks any immunoreactivity for parvalbumin, clearly distinguishing it from the underlying lateral posterior-pulvinar complex. In addition, the relay neurons within this nucleus occurred at a slightly higher density than that seen in the lateral posterior-pulvinar complex, which was also striated in appearance (see above). No subdivisions of the lateral dorsal nucleus were obvious with the stains used in the current study.

\section{Ventral Thalamus}

The ventral thalamus is typically defined as being composed of the reticular nucleus, the zona incerta and the ventral lateral geniculate nucleus. Jones [2007] includes the nucleus of the field $(\mathrm{H})$ of Forel in the ventral thalamus but excludes the subthalamic nucleus on the basis of its different embryological origin. In the current description, for the sake of completeness, all of the aforementioned structures will be described as part of the ventral thalamus [while keeping in mind the reasons for the inclusion of the field $(\mathrm{H})$ of Forel and the exclusion of the subthalamic nucleus by Jones, 2007] (online suppl. fig. A-J). In the elephant, the reticular nucleus was observed to surround the nuclei of the dorsal thalamus as commonly seen across mammals [Jones, 2007]. The reticular nucleus was observed to cover the rostral pole of the dorsal thalamic nuclear mass and spread as a thin sheet, approximately $1-1.5 \mathrm{~mm}$ thick, around the lateral aspect of the dorsal thalamic nuclear mass. The reticular nucleus was observed to wane and then disappear at the rostrocaudal level of the lateral geniculate nucleus. The nucleus contained moderate size, intensely stained (for Nissl substance), mostly bipolar (some multipolar), triangular shaped neurons. The packing density of these neurons was quite low in comparison to other mammals. All of the neurons in the reticular nucleus were immunopositive for parvalbumin but immunonegative for all other immunostains used in the current study. The ventromedial aspect of the reticular nucleus was found adjacent to the zona incerta, which was readily distinguished from the
96 
reticular nucleus by its smaller and less intensely stained neurons. No immunohistochemical stains used in the current study labelled the neurons of the zona incerta. In the more caudal aspects of the zona incerta, dorsal and ventral subdivisions, split by a thin myelinated lamina, were apparent. The ventral zona incerta subdivision exhibited a neuropil immunopositive for calbindin, while the dorsal zona incerta subdivision was not immunoreactive for any of the stains used in the current study. The ventral zona incerta subdivision did not show any continuity with the reticular nucleus as observed for the dorsal zona incerta subdivision. Ventral and medial to the zona incerta, lateral to the ventral aspect of the hypothalamus and dorsal to the subthalamic nucleus, an ill-defined region of large, loosely packed multipolar neurons was designated as the field $(\mathrm{H})$ of Forel. A low number of smaller neurons were immunopositive for calbindin within this region, but otherwise no clear immunostaining was evident.

A distinct ventral lateral geniculate nucleus was observed on the lateral and ventral edge of the lateral geniculate nucleus. Within this nucleus neurons immunopositive for parvalbumin, calbindin and calretinin were observed, as well as neuropil immunopositive for these three antibodies. The ventral lateral geniculate nucleus was separated from the lateral geniculate nucleus by the thin infrageniculate leaflet, which was invested with a moderate density of orexin-A-immunopositive axons and axon terminals. The subthalamic nucleus was located between the zona incerta/fields of Forel and the internal capsule/cerebral peduncle in the elephant, a position typical of that seen in mammals. It was a large nucleus, with a maximum mediolateral extent of approximately $15 \mathrm{~mm}$, a rostrocaudal extent a little over $10 \mathrm{~mm}$, and a dorsoventral thickness of approximately $2.5 \mathrm{~mm}$. Within this nucleus large, moderately densely packed, intensely stained (for Nissl substance), multipolar neurons were observed. The majority of the immunostaining undertaken herein did not label any neurons in this nucleus, but a small cluster of smaller neurons were immunoreactive for both calbindin and calretinin in the very medial and ventral portion of this nucleus where it abutted the hypothalamus.

\section{Epithalamus}

In the adult mammalian brain the epithalamus is considered to be composed of the anterior and posterior paraventricular nuclei, the medial and lateral habenular nuclei, the stria medullaris thalami and the pineal body [Jones, 2007]. The pineal body was unfortunately lost during the preparation of the tissue used in the current

Elephant Diencephalon and Brainstem study [Manger et al., 2009] and therefore will not be described herein [but see Shoshani et al., 2006, for details of the presence/absence and histological appearance of the pineal gland in elephants]. Jones [2007] also points out that the pretectum forms part of the pronuclear mass that gives rise to the epithalamus during development, and thus this portion of the elephant diencephalon is described here (online suppl. fig. A-J).

A thin band of densely packed, moderate sized, intensely stained (for Nissl substance) neurons was evident medial to the rostral half of the dorsal thalamic nuclear mass, interposed between this mass and the lateral wall of the third ventricle. This band of cells was observed to extend from the dorsal aspect of the diencephalon in a ventral direction for approximately $10 \mathrm{~mm}$. This band of neurons, presumably the paraventricular nuclei, was invested with a high density of orexin-A-immunopositive axons and axon terminals. The paraventricular nuclei are commonly divided into anterior and posterior nuclei in mammals [Jones, 2007], but we could not find evidence for these divisions in the elephant. Dorsal and caudal to the dorsal-most aspect of the paraventricular nuclei, in the dorsal medial aspect of the diencephalon, the habenular complex was observed. Within the habenular complex distinct medial and lateral nuclei were observed, with the medial habenular nucleus evincing densely packed neurons immunopositive for choline acetyltransferase. No immunoreactivity to any of the stains used in the current study was observed in the lateral habenular nucleus and no clear habenular commissure was evident. A distinct fasciculus retroflexus, also immunopositive for choline acetyltransferase, was seen to emerge from the ventral aspect of the habenular nuclei and describe a course lateral to the rostral-most parts of the periaqueductal grey matter, terminating in the small interpeduncular nucleus in the ventral midline of the midbrain. Dorsal, lateral and rostral to the habenular complex a distinct, heavily myelinated stria medullaris thalami was observed on the dorsomedial surface of the diencephalon. In the more rostral portions of the stria medullaris thalami a distinct nuclear mass, separated from the dorsal thalamic nuclear mass by the stria medullaris thalami, was invested into the dorsal aspect of the stria. Within this nucleus, which we term the nucleus of the stria medullaris thalami, a high density of neurons immunopositive for both calbindin and calretinin was observed.

A distinct pretectal area was observed to lie rostral to the superior colliculi and caudal to the dorsomedial aspect of the dorsal thalamic nuclear mass. This moderately large region, about $5 \mathrm{~mm}$ in diameter, was occupied 
by a low to moderate density of calbindin-immunoreactive neurons and a high density of calretinin-immunopositive neurons. The pretectal region is thought to have several distinct nuclei, but subdivisions of this region were not evident in the preparations made in the current study; rather, this region appeared to be a homogenous nuclear mass. Further study with different antibodies may reveal a more complex organization of this area in the elephant.

\section{Hypothalamus}

As observed previously [Diepen et al., 1956], the hypothalamus of the African elephant conforms to the general pattern observed across mammalian species. It occupied the ventral part of the diencephalon, with a distinct hypothalamic sulcus demarcating it from the remainder of the diencephalon (online suppl. fig. A-G). The caudal pole of the third ventricle separated the left and right hypothalami, while the passage of the fornix clearly demarcated the medial and lateral hypothalamic zones. The lateral hypothalamic zone was architectonically homogeneous, but the immunohistochemical stains used in the current study (see below) allowed the identification of distinct cholinergic, catecholaminergic and orexinergic nuclei within this zone. The medial zone, while also not easy to parcellate using architectonic methods, was composed of supraoptic, tuberal and mammillary regions. Within the supraoptic region a large and distinct supraoptic nucleus was apparent and this nucleus appeared to occupy the floor of the hypothalamus from the midline to the lateral edge. The cells within the supraoptic nucleus were darkly stained for Nissl substance and were also strongly immunoreactive for the calbindin antibody. The paraventricular nuclei of the supraoptic region were not readily distinguished from the remainder of the medial hypothalamic nuclei using cytoarchitecture, but calbindin and tyrosine hydroxylase immunoreactivity did reveal distinct paraventricular nuclei (calbindin), with the A14 catecholaminergic group (tyrosine hydroxylase, see below) forming a notable portion of the paraventricular nuclei.

The tuberal region of the hypothalamus extended across both the medial and the lateral zones of the hypothalamus. We could identify both the ventromedial hypothalamic and the arcuate nuclei within the medial hypothalamic zone of the tuberal region. The ventromedial hypothalamic nucleus was delineated on the basis of intensely Nissl-stained and densely packed cells, but none of the immunohistochemical stains employed in the present study were reactive to any of these cells. The ventro- medial hypothalamic nucleus occupied a position that can be considered typical for the mammalian hypothalamus. Ventral and medial to this nucleus the arcuate nucleus was observed to form a thin sheet around the floor of the third ventricle. Within the arcuate nucleus numerous tyrosine-hydroxylase-immunoreactive neurons, forming the A12 group (see below), were observed. Again, the location of the arcuate nucleus is typical of the mammalian hypothalamus. The mammillary region, as a whole, was relatively small in the elephant, with distinct nuclei being difficult to discern on the basis of the stains used in the current study. A small, flattened region of cells occupying the base of the hypothalamus, below the arcuate nuclei, likely represents the mammillary nuclei, but we could not discern nuclear subdivisions within this region. Arising from this region the mammilothalamic tract could be observed to course dorsally through the hypothalamic grey matter, medial to the zona incerta, and continue towards the anterior nuclei of the dorsal thalamus (online suppl. fig. A, C, F). A distinct posterior hypothalamic nucleus could not be identified with certainty; however, an ill-defined group of cells that may correspond to this nucleus was noted.

\section{Cholinergic Neurons within the Hypothalamus}

Within the hypothalamus three distinct clusters of choline acetyltransferase $(\mathrm{ChAT}+)$ neurons were observed (online suppl. fig. D). These were the dorsal, lateral and ventral cholinergic hypothalamic groups, but they were restricted to a small region within the hypothalamus, covering a rostrocaudal distance of approximately $5 \mathrm{~mm}$. The dorsal hypothalamic group was located within the dorsomedial aspect of the hypothalamus, caudal to the fornix, in the dorsal hypothalamic area. The ChAT+ neurons of this nucleus exhibited a sparse density and had round to fusiform somas that showed no specific dendritic orientation. The lateral hypothalamic group was found within the dorsolateral portion of the hypothalamus, caudolateral to the fornix, in the most dorsal aspect of the lateral hypothalamic area, adjacent to the zona incerta. The ChAT+ neurons within this nucleus were low in density and possessed cell bodies that were ovoid in shape and bipolar in type with no specific dendritic orientation. The ventral hypothalamic group was located in the ventral medial portion of the hypothalamus, ventral and lateral to the arcuate nucleus in a location above the optic chiasm. The ChAT+ neurons in this nucleus were found in a moderate to low density and the neurons had somal bodies that were round in shape and bipolar in type with a roughly medio-
Maseko/Patzke/Fuxe/Manger 
lateral dendritic orientation. In all cases the cholinergic neurons of the elephant hypothalamus were palely immunostained.

\section{Catecholaminergic Neurons within the}

Hypothalamus

Six clusters of tyrosine-hydroxylase-immunopositive $(\mathrm{TH}+)$ neurons, presumably catecholaminergic, forming distinct nuclei were observed within the elephant hypothalamus, these being: the anterior hypothalamic group, dorsal division (A15d); the anterior hypothalamic group, ventral division $(\mathrm{A} 15 \mathrm{v})$; the rostral periventricular cell group (A14); the zona incerta group (A13); the tuberal cell group (A12), and the caudal diencephalic group (A11) (online suppl. fig. B-G). Within the dorsal rostral portion of the hypothalamus, dorsal and medial to the fornix, a low to moderate density of $\mathrm{TH}+$ neurons was assigned to the A15d nucleus. The cell bodies of these neurons were ovoid in shape and bipolar in type but did not exhibit any specific dendritic orientation. The A15v nucleus was comprised of a low density of $\mathrm{TH}+$ neurons in the ventrolateral portion of the hypothalamus, just above the supraoptic nucleus. The cell bodies of these neurons were ovoid in shape and bipolar in type with a dendritic orientation running roughly parallel to the dorsal margin of the supraoptic nucleus. $\mathrm{TH}+$ neurons forming the A14 nucleus were observed to form bilateral low-density columns adjacent to the lateral edges of the third ventricle in the rostral two thirds of the hypothalamus. The cell bodies, which were low in number in this nucleus, were ovoid in shape and predominantly bipolar in type with some multipolar neurons and showed a dendritic orientation that ran parallel to the wall of the third ventricle. A moderate number of $\mathrm{TH}+$ neurons assigned to the A13 nucleus were located within the dorsolateral aspect of the hypothalamus, lateral to the fornix, and extended into the white matter below the zona incerta. These neurons formed a roughly triangular shaped nucleus with the point of the triangle extending into the region below the zona incerta. These neurons had cell bodies that were either ovoid or polygonal in shape and bipolar or multipolar in type, with a roughly mediolateral dendritic orientation. The $\mathrm{TH}+$ neurons forming the A12 nucleus were found in the ventral medial portion of the caudal third of the hypothalamus, surrounding and below the floor of the third ventricle in the area of the arcuate nucleus, but did not extend lateral to this region as seen in the rat [Hökfelt et al., 1976]. The TH+ neurons within this nucleus had an ovoid and bipolar somal morphology with a dendritic orientation parallel to the wall of the third ventricle. A low density of $\mathrm{TH}+$ neurons assigned to the A11 nucleus was observed within the medial hypothalamic grey matter adjacent to the caudal pole of the third ventricle. These neurons were ovoid in shape, had larger somas than the neurons forming the other catecholaminergic nuclei of the hypothalamus, were a mixture of bipolar and multipolar types and showed no clear dendritic orientation.

\section{Orexinergic Neurons within the Hypothalamus}

Orexinergic neurons were found within the hypothalamus and in closely adjacent areas (online suppl. fig. B-F). These neurons could be readily separated into two distinct neuronal types based on somal size, these being the parvocellular and magnocellular orexinergic immunopositive $(\mathrm{OxA}+)$ groups (fig. 5). The magnocellular group could be further subdivided into three distinct nuclei: a main cluster in the perifornical and lateral hypothalamus, a cluster associated with the zona incerta and a cluster associated with the optic tract. The parvocellular neurons were found in the medial hypothalamus but could not be subdivided into separate nuclei, rather forming a topologically continuous cluster. Orexinergic neurons were observed to span a rostrocaudal distance of approximately $20 \mathrm{~mm}$ in the elephant and were seen as far as $5 \mathrm{~mm}$ from the wall of the third ventricle.

The parvocellular OxA+ group was located in the medial zone of the hypothalamus between the wall of the third ventricle and the fornix, mainly in a paraventricular position. Rostrally these $\mathrm{OxA}+$ neurons were found in the dorsomedial aspect of the hypothalamus, and caudally these neurons were located in approximately at the middorsoventral level of the hypothalamus. These parvocellular OxA+ neurons were found in a moderate to high density throughout the regions in which they were localized and no distinct topological discontinuities were observed; thus, they appear to form a single cluster extending as a column along the rostrocaudal axis of the hypothalamus. The cell bodies were ovoid in shape and the majority of the neurons were clearly bipolar, but they showed no specific dendritic orientation and were observed to lie within a moderately dense plexus of OxA+ varicose nerve terminals.

A substantial number of magnocellular $\mathrm{OxA}+$ neurons observed throughout the perifornical and the entire lateral zone of the hypothalamus were assigned to the main orexinergic cluster $(\mathrm{Mc})$. A small to moderate number of $\mathrm{OxA}+$ neurons were found in a ventrolateral position within the hypothalamus, close to the optic tract, and these neurons were assigned to the optic tract cluster 


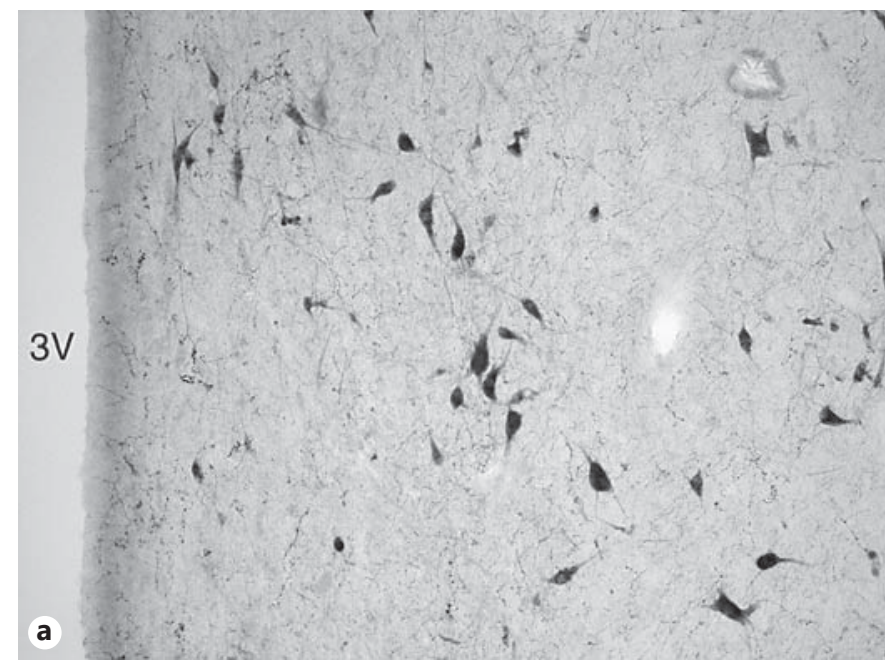

Fig. 5. Photomicrographs of orexin-A-immunopositive neurons in the medial hypothalamus (a) and the lateral hypothalamus (b) showing the mixed parvocellular and magnocellular cells (a) close to the wall of the third ventricle $(3 \mathrm{~V})$ and the mostly magnocellular

(OTc). Dorsolaterally, at the rostral and mid-levels of the hypothalamus, a small number of OxA+ neurons were found to extend laterally from the main cluster out of the hypothalamus into the fields of Forel and the zona incerta, and these neurons formed the zona incerta cluster (Zic). The magnocellular OxA+ neurons exhibited a variety of somal shapes, and some were bipolar while the majority appeared multipolar. No clear dendritic orientation was observed in the main and optic tract neuronal clusters, but the OxA+ neurons found in the zona incerta cluster were clearly bipolar and exhibited a dendritic orientation coincident with the mediolateral orientation of the zona incerta.

\section{Brainstem}

The general structure of the brainstem of the African elephant, herein referring to the three subdivisions of midbrain, pons and medulla oblongata, resembles that seen in most other mammals. In the current study, for ease of description and interpretation, the brainstem is considered to be composed of five distinct basic components: the cranial nerve nuclei and associated nerves, ascending and descending fibre pathways, neuromodulatory nuclei (cholinergic, catecholaminergic, and serotonergic nuclei), intrinsic nuclei (contained within one part of the brainstem), and tegmental/reticular nuclei. Moreover, our description is based on a template drawn from the atlas of the laboratory rat brain [Paxinos et al.,

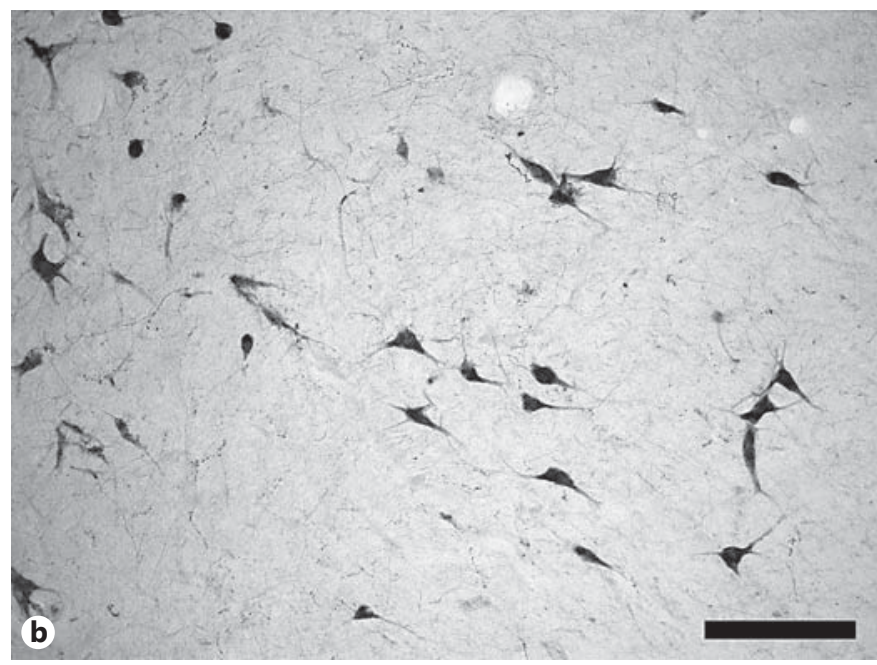

neurons of the main orexinergic cluster (b) in the lateral hypothalamic area. Medial is to the left and dorsal to the top. Scale bar in $\mathbf{b}=250 \mu \mathrm{m}$ and applies to both.

2009], but where the architecture differs we relied on descriptions of other species for both nomenclature and clarity (i.e. while the laboratory rat provides a comprehensive template of the mammalian brainstem, it does not always cover all aspects of mammalian brainstem anatomical variation). While some structures do cross the borders between the major subdivisions of the brainstem, many are found completely within one of these subdivisions. The appearance of those structures that occur in more than one major subdivision of the brainstem is described in the section relating to each subdivision, with reference to the same structure in the adjacent subdivision.

\section{Midbrain}

The Cranial Nerve Nuclei of the Midbrain

Within the midbrain of the African elephant we were able to readily identify four distinct nuclei associated with specific cranial nerves: the oculomotor nucleus, the Edinger-Westphal nucleus, the trochlear nucleus and the trigeminal mesencephalic nucleus. All of these nuclei were located in regions of the midbrain that can be considered typical of mammals.

The Oculomotor Nucleus (III). The cells comprising the bilaterally paired oculomotor nuclei were found in the ventral medial aspect of the periaqueductal grey matter in a para-raphe position (online suppl. fig. K, L). The oculomotor nucleus had a maximum diameter of approximate- 
ly $3 \mathrm{~mm}$ and a rostrocaudal length of approximately $8 \mathrm{~mm}$. The cells comprising this nucleus have the appearance typical of large motor neurons seen in other mammals and were all immunopositive for ChAT. The large oculomotor nerve emerged from the ventral portion of the nucleus, passed through the medial aspect of the midbrain tegmentum, and exited the midbrain at the medial aspect of the cerebral peduncle as is typically seen in other mammals.

The Preganglionic Component of the Edinger-Westphal Nucleus $(E W)$. Unlike the anatomy often seen in other mammals, the neurons assigned to the preganglionic component of the Edinger-Westphal nucleus [termed EWpg by Kozicz et al., 2011] formed two distinct pararaphe columns medial to the oculomotor nucleus (online suppl. fig. K). The ChAT-immunopositive neurons that formed these bilateral columns had the appearance of typical multipolar motor neurons, but they were smaller than those seen in the oculomotor nucleus, being of medium rather than large size. The ChAT-immunopositive axons that emanated from these neurons joined the oculomotor nerve to exit the brain in the same location as the oculomotor nerve.

The Trochlear Nucleus (CN VI). At the level of the caudal pole of the oculomotor nucleus, a second cluster of large ChAT-immunopositive motor neurons was observed. This cluster of neurons was located outside, but adjacent to, the periaqueductal grey matter in the dorsal medial aspect of the midbrain tegmentum in a position immediately lateral to the medial longitudinal fasciculus and was designated as the trochlear nucleus (online suppl. fig. M, N). The cross-sectional coronal dimensions of this nucleus were slightly smaller than the oculomotor nucleus, but it exhibited a similar rostrocaudal length. While we could not observe the passage of the trochlear nerve in our preparation, a previous report indicates that it exits as a relatively slender nerve on the dorsal aspect of the midbrain as seen in other mammals [Shoshani et al., 2006].

The Midbrain Portion of the Trigeminal Mesencephalic Nucleus (mesV) and Tract (mesVtr). A low-density cluster of large spherical ganglion cells found on the lateral edge of the periaqueductal grey matter, as is typical for most mammals, was assigned to the trigeminal mesencephalic nucleus (online suppl. fig. K-P). These cells were clearly revealed with the Nissl staining but were not immunopositive for any of the markers used in the current study. The trigeminal mesencephalic tract was found immediately lateral and adjacent to the edge of the periaqueductal grey matter and was ob- served to arise from the trigeminal nerve at the level of the pons (see below). This nucleus and tract form a rostral continuation of the same nucleus and tract observed in the pons.

The Ascending and Descending Fibre Pathways of the Midbrain

Within the midbrain of the elephant, several distinct fibre bundles carrying both descending motor and ascending sensory information were evident. These included the medial longitudinal fasciculi, the medial and lateral lemnisci, and the corticobulbar and corticospinal tracts that formed the cerebral peduncle. The medial longitudinal fasciculi were evident as a bilateral pair of tracts located in the dorsomedial aspect of the midbrain tegmentum. These paired fasciculi, measuring approximately $2 \mathrm{~mm}$ in diameter, became evident at the level of the oculomotor nuclei and were observed throughout the midbrain caudal to this level, in the same location, to continue into the pons (see below) (online suppl. fig. L-P). The medial lemniscus was observed to coalesce as a distinct entity around the longitudinal fasciculus of the pons, and as it was followed rostrally within the midbrain it formed a distinct tract lateral to the midbrain tegmentum (online suppl. fig. G-P). The medial lemniscus was observed to be in a more dorsal location in this lateral aspect of the midbrain towards the rostral portions of the midbrain, presumably making a passage towards the somatic sensory nuclei of the dorsal thalamus. The lateral lemniscus was observed to coalesce as a distinct fibre tract lateral to the longitudinal fasciculus of the pons, and take a dorsal course within the most lateral aspect of the midbrain, terminating in the inferior colliculi (online suppl. fig. M-P). Occasional small clusters of neurons within the lateral lemniscus were identified as nuclei related to this lemniscus. Both the medial and the lateral lemnisci were of similar size, being approximately $3 \mathrm{~mm}$ at their widest cross-sectional diameter.

The corticobulbar and corticospinal tracts of the elephant were not readily distinguishable in our preparation; thus, they are grouped together within the cerebral peduncle (crus cerebri) in the current description. The cerebral peduncle was observed to be a distinct continuation of the internal capsule and occupied the ventrolateral aspect of the full length of the midbrain (online suppl. fig. E-P). The cerebral peduncle was large, expanding up to almost $10 \mathrm{~mm}$ in cross-sectional dimensions at certain points within the midbrain. Many of these descending motor fibres were observed to terminate in the ventral pontine nucleus (see below), with the remainder forming 
the longitudinal fasciculus of the pons that terminated in cranial nerve nuclei or continued as the pyramidal tract in the medulla oblongata (see below). No differences of distinction were observed for the elephant cerebral peduncle when compared to that observed in other mammals.

\section{The Neuromodulatory Nuclei of the Midbrain}

The Catecholaminergic Nuclei of the Midbrain. Within the elephant midbrain, the tyrosine-hydroxylase-immunopositive $(\mathrm{TH}+)$ neurons, forming distinct catecholaminergic nuclei, were found within the ventral tegmental area (the A10 complex, including the A10, A10c, A10d and A10dc nuclei), the substantia nigra (the A9 complex, including the $\mathrm{A} 9 \mathrm{pc}, \mathrm{A} 9 \mathrm{l}, \mathrm{A} 9 \mathrm{v}$ and $\mathrm{A} 9 \mathrm{~m}$ nuclei) and the retrorubral nucleus (A8) within the midbrain tegmentum. For the most part these nuclei appeared to be arranged in a manner consistent with that observed in other mammals; however, the pars compacta portion of the substantia nigra (A9pc) showed a distinct and unusual arrangement.

The Ventral Tegmental Area Nuclei (VTA, A10 Complex). A nuclear complex containing four nuclei (A10 ventral tegmental area; $A 10 c$ - ventral tegmental area, central; A10d - ventral tegmental area, dorsal, and A10dc - ventral tegmental area, dorsal caudal) was found in the medial portion of the tegmentum of the midbrain (online suppl. fig. G-O). These nuclei extend from within the periaqueductal grey matter around the base of the aqueduct, into the tegmentum below the periaqueductal grey matter around the midline, through to and around the interpeduncular nucleus. The A10 nucleus consisted of a moderate to high density of $\mathrm{TH}+$ neurons found rostral, dorsal and dorsolateral to the interpeduncular nucleus and was bound laterally by the oculomotor nerve. These neurons were ovoid in shape and bipolar in type, with the dendrites orientated parallel to the edge of the interpeduncular nucleus. The A10c nucleus was comprised of a dense cluster of ovoid, bipolar $\mathrm{TH}+$ neurons located rostral to the decussation of the superior cerebellar peduncle and ventral to the nucleus ellipticus. The dendritic orientation of these neurons was parallel to the dorsal margin of the interpeduncular nucleus or the ventral margin of the nucleus ellipticus. Caudal to the nucleus ellipticus, a moderately dense bilateral parasagittal cluster of ovoid and bipolar neurons with a dorsoventral dendritic orientation was observed immediately dorsal to A10c, between it and the oculomotor nucleus. These parasagittal columns of $\mathrm{TH}+$ neurons were assigned to the A10d nucleus. A low density of small $\mathrm{TH}+$ neurons, constituting the A10dc subdivi- sion, was found within the periaqueductal grey matter adjacent to and surrounding the ventral half of the cerebral aqueduct. The cell bodies of these neurons were ovoid in shape, a mixture of bipolar and multipolar types with the dendrites orientated parallel to the edge of the cerebral aqueduct.

The Substantia Nigra Complex (A9). The substantia nigra nuclear complex was located in the ventral and lateral portions of the midbrain tegmentum, lying just dorsal to the cerebral peduncles, but in the elephant it appears to be far more extensive than seen in many other mammals studied to date. The four distinct nuclei evident within the A9 complex included: the substantia nigra, pars compacta (A9pc); the substantia nigra, ventral (A9v); the substantia nigra, pars lateralis (A91), and the substantia nigra, pars medialis (A9m) (online suppl. fig. G-O). The A9pc was seen as dense tightly packed, elongated islands of $\mathrm{TH}+$ neurons of different sizes that not only occurred in the region where the classical pars compacta is found in mammals but also extended into the lateral aspect of the midbrain tegmentum, ascending as isolated islands of $\mathrm{TH}+$ cells forming an arc that passed lateral to the red nucleus, and continued through to the ventrolateral periaqueductal grey matter (fig. 6). In each of the islands of A9pc, dense clusters of $\mathrm{TH}+$ cells were surrounded by dense $\mathrm{TH}+$ neuropil staining originating from the $\mathrm{TH}+$ cells within the islands, making these islands very distinct. These islands of A9pc cells could also be readily identified in the Nissl-stained material. The neurons were ovoid in shape and bipolar in type, showing no distinct dendritic orientation within the island. A number of $\mathrm{TH}+$ neurons that were ovoid in shape and bipolar in type with no clear dendritic orientation found within the dorsal portion of the cerebral peduncle ventral to $\mathrm{A} 9 \mathrm{pc}$ were designated as the $\mathrm{A} 9 \mathrm{v}$ nucleus. At the lateral edge of A9pc, adjacent to the dorsal lateral margin of the cerebral peduncle, a loose aggregation of ovoid, bipolar $\mathrm{TH}+$ neurons formed the A9l nucleus. The neurons within this nucleus were moderate in density and showed no specific dendritic orientation. A dense cluster of numerous $\mathrm{TH}+$ neurons forming the A9m nucleus was found medial to A9pc and lateral to the root of the oculomotor nerve, at the dorsal medial margin of the cerebral peduncle. These neurons were ovoid in shape and bipolar in type and exhibited no specific dendritic orientation.

The Retrorubal Nucleus (A8). A sparsely packed but relatively numerous cluster of $\mathrm{TH}+$ neurons assigned to the A8 nucleus were found scattered throughout the ventral-most aspect of the midbrain tegmentum, in a position ventrolateral and caudal to the magnocellular divi- 

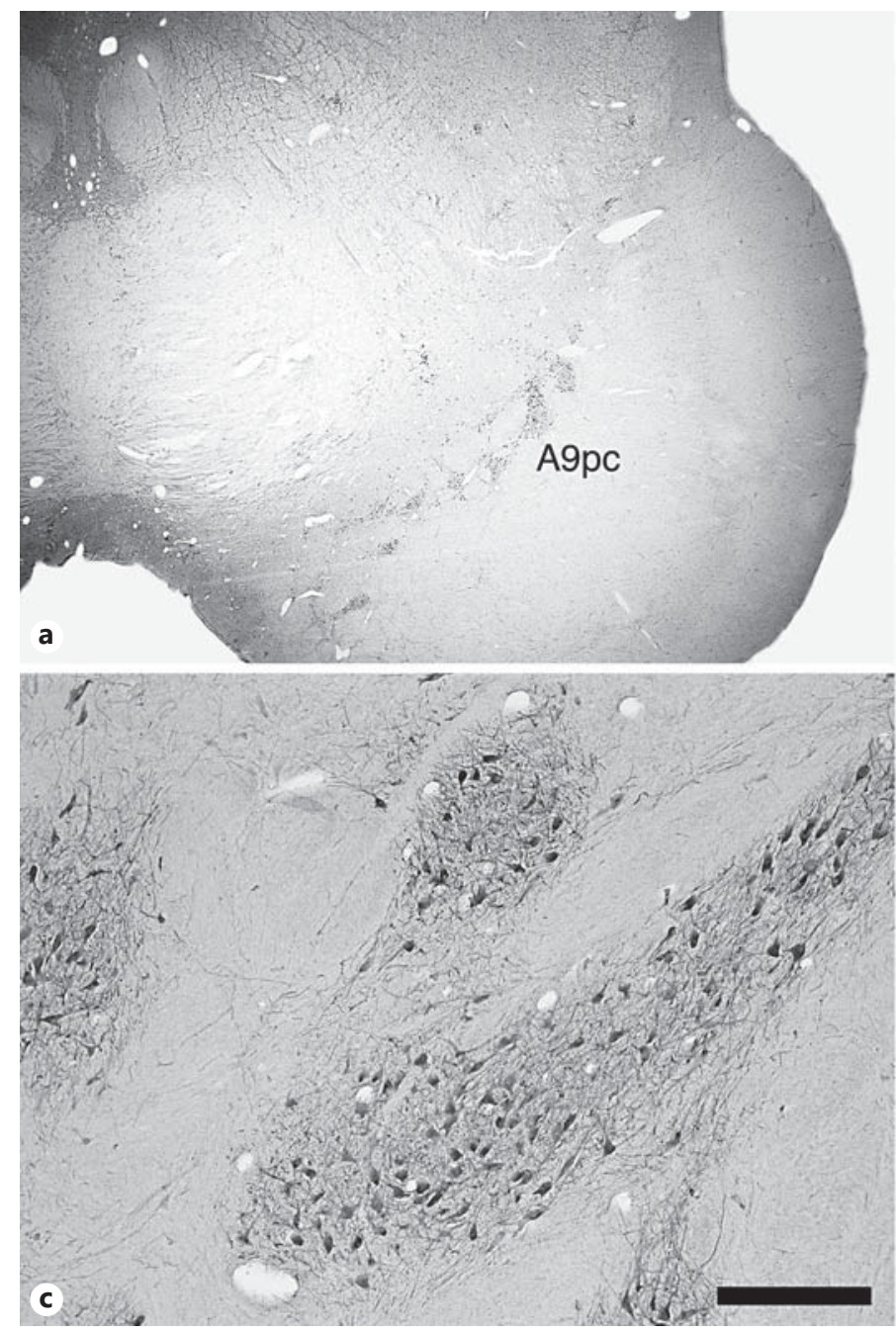

Fig. 6. Photomicrographs of the clustered organization of the pars compacta portion of the substantia nigra (A9pc). a Low-power Nissl stain showing the presence of intensely stained islands of neurons forming the A9pc. $\mathbf{b}$ Tyrosine-hydroxylase-immunoreactive neurons demonstrating that the islands visible in the Nissl stain are catecholaminergic. Scale bar in $\mathbf{b}=5 \mathrm{~mm}$ and applies to $\mathbf{a}$ and $\mathbf{b}$. c Higher-power photomicrograph of the tyrosine-hydrox-

sion of the red nucleus and dorsal to the A9 complex (online suppl. fig. $\mathrm{K}-\mathrm{N}$ ). The cells that formed this nucleus were mostly ovoid in shape, a mixture of bipolar and multipolar types, and showed no specific dendritic orientation.

The Serotonergic Nuclei of the Midbrain. The serotonergic immunoreactive neurons $(5 \mathrm{HT}+$ ) identified in the current study of the elephant formed nuclei that were the same as those previously identified in all other eutherian mammals studied to date [e.g. Maseko et al., 2007; Dell et al.,
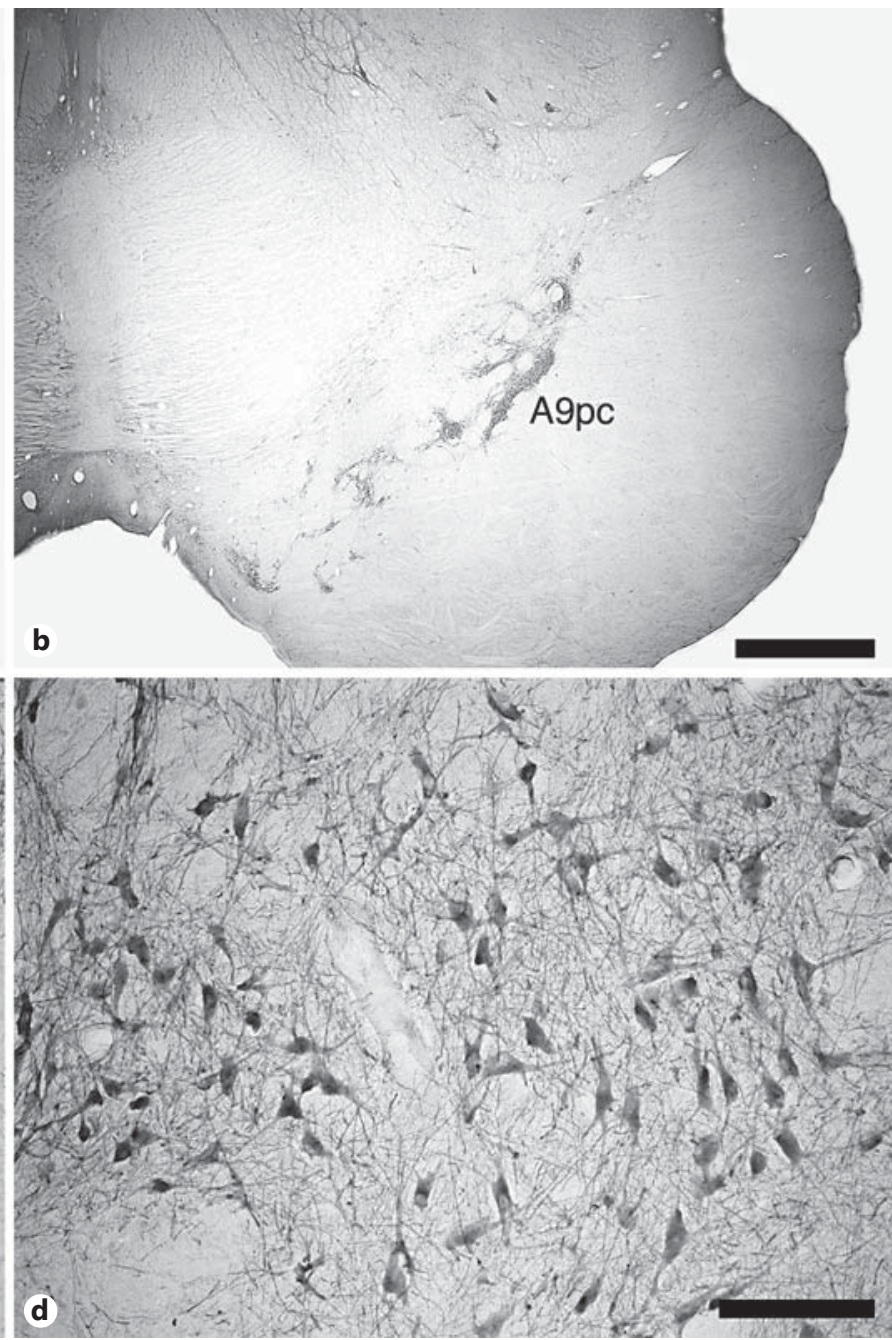

ylase-immunoreactive neurons showing the clustered arrangement of the A9pc. Scale bar $=500 \mu \mathrm{m}$. d Higher-power photomicrograph of the tyrosine-hydroxylase-immunoreactive neurons showing the dense immunopositive neuropil within the islands of A9pc neurons. Scale bar $=250 \mu \mathrm{m}$. a-d Medial is to the left and dorsal to the top.

2010; Kruger et al., 2012]. The serotonergic nuclei identified in the midbrain of the elephant were seen to form what is commonly termed the rostral serotonergic nuclear cluster [Bjarkam et al., 1997]. This cluster contained a number of distinct nuclei that were found from the level of the decussation of the superior cerebellar peduncle through to the rostral-most levels of the pons (see below).

The Caudal Linear Nucleus (CLi). The 5HT+ neurons forming this nucleus were the most rostrally located of the serotonergic neurons. A low to moderate density of 
$5 \mathrm{HT}+$ neurons forming a cluster was found around the midline of the ventral midbrain tegmentum immediately dorsal and rostral to the interpeduncular nucleus in a position just rostral and ventral to the decussation of the superior cerebellar peduncle (online suppl. fig. K-M). These cell bodies were ovoid in shape and bipolar with a dorsoventral dendritic orientation.

The Supralemniscal (B9) Nucleus. The neurons forming this nucleus appeared to be a lateral extension of the neuronal cluster comprising the most ventral portion of $\mathrm{CLi}$ (online suppl. fig. K, L). The low density of 5HT+ B9 neurons was located ventrolateral to the red nucleus, in a position just caudal to the oculomotor nerve, and extended as an arc of neurons a relatively small distance into the medial portion of the midbrain tegmentum. The cell bodies of these neurons were ovoid and bipolar and showed a rough mediolateral dendritic orientation.

The Median Raphe (MnR) Nucleus. The median raphe nucleus was characterized by two distinct, densely packed $5 \mathrm{HT}+$ neuronal columns on either side of the midline in a para-raphe position dorsal and caudal to the decussation of the superior cerebellar peduncle (online suppl. fig. $\mathrm{M}-\mathrm{P}$ ). The rostral limit of this nucleus was coincident with the caudal level of the oculomotor (III) nuclei, and the caudal limit of this nucleus was within the rostral portion of the pons (see below). The cell bodies of the neurons were ovoid in shape and bipolar and had a mostly dorsoventral orientation of the dendrites.

The Dorsal Raphe (DR) Nuclear Complex. The six nuclei forming the $5 \mathrm{HT}+$ dorsal raphe nuclear complex included: the dorsal raphe interfascicular (DRif) nucleus, the dorsal raphe ventral (DRv) nucleus, the dorsal raphe dorsal (DRd) nucleus, the dorsal raphe peripheral (DRp) nucleus, the dorsal raphe lateral (DRl) nucleus, and the dorsal raphe caudal (DRc) nucleus (online suppl. fig. L-P). These six nuclei were found, for the most part, within the periaqueductal and periventricular grey matter from the level of the oculomotor nuclei to the level of the trigeminal motor nucleus, spanning a rostrocaudal extent of approximately $30 \mathrm{~mm}$. DRif contained a moderate density of $5 \mathrm{HT}+$ neurons that were located between the two medial longitudinal fasciculi. These neurons were ovoid in shape and bipolar with a dorsoventral dendritic orientation. A high density of 5HT+ neurons forming the DRv nucleus was found immediately dorsal to DRif, within the medial ventral portion of the periaqueductal grey matter, just caudal to the oculomotor nuclei. These neurons had a neuronal morphology similar to that of DRif but exhibited a range of dendritic orientations. The DRd nucleus was formed by a high-density cluster of $5 \mathrm{HT}+$ neurons immediately dorsal to DRv and ventral to the inferior border of the cerebral aqueduct in the medial portion of the periaqueductal grey matter. The neuronal morphology was similar to that of DRif and DRv but with a mediolateral dendritic orientation. There appeared to be some indication of this nucleus being bilaterally paired; however, this was dependent on the rostrocaudal level observed. In the ventrolateral portion of the periaqueductal grey matter lateral to the DRd and DRv were $5 \mathrm{HT}+$ neurons representing the DRp nucleus. Several neurons were found in the adjacent midbrain tegmentum and were the only ones found outside the periaqueductal grey matter. A moderate to low density of these neurons was located within the periaqueductal grey matter and a lower density of more scattered neurons was observed in the tegmental region. The DRp $5 \mathrm{HT}+$ neurons were ovoid, marginally larger than those of DRd, DRv and DRif, and bipolar and showed no specific dendritic orientation. The $5 \mathrm{HT}+$ neurons of the DRl were located dorsolateral to DRd and adjacent to the ventrolateral edges of the cerebral aqueduct. The neurons of this nucleus were readily distinguished from the rest of the dorsal raphe nuclei as the somas were larger and the neurons showed a mixture of bipolar and multipolar types. These neurons were found in a moderate density and showed a dendritic orientation that was roughly parallel to the wall of the cerebral aqueduct. As the DRl was followed caudally, the cerebral aqueduct opened in the fourth ventricle and the DRd, DRv and DRif nuclei disappeared. At this level the neurons of DRl formed an arc across the midline of the dorsal portion of the periventricular grey matter. This caudal arc of the DRl was classified as the DRc nucleus, and this continued for some distance into the periventricular grey matter of the pons (see below). The neuronal morphologies of the neurons in DRc and DRl were identical, but due to a lack of 5HT+ neurons in this region in the brain of monotremes [Manger et al., 2002] DRc was classified as an independent nucleus.

\section{The Intrinsic Nuclei of the Midbrain}

The Superior Colliculus. The superior colliculi, which evinced a cortical morphology like that described in other mammals, formed the rostral portion of the midbrain tectum (online suppl. fig. $\mathrm{K}-\mathrm{N}$ ). The superior colliculus was a large structure, with mediolateral dimensions of almost $15 \mathrm{~mm}$ at its widest, dorsoventral dimensions of over $10 \mathrm{~mm}$ at its deepest, and rostroventral dimensions of $15 \mathrm{~mm}$. Within the superior colliculus seven layers could be identified, and these are, from dorsal to ventral: 
the optic tract layer (OT), the zonal layer (Zo), the superficial grey matter layer $(\mathrm{SuG})$, the optic nerve layer (OP), the intermediate grey layer $(\operatorname{InG})$, the deep grey layer (DpG), and the deep white matter layer (DpWh). The Zo and InG grey matter layers of the superior colliculus exhibited neuropil and neurons immunoreactive for parvalbumin. All superior collicular layers possessed both neurons and neuropil immunopositive for both calbindin and calretinin, although the density of immunopositive neurons appeared to be greatest in the InG and DpG for both calbindin and calretinin. The DpWh layer appeared to give rise to large fibre tracts that coalesced medially to form the distinct commissure of the superior colliculus (csc) which crossed the midline rostral to the commissure of the inferior colliculus and dorsal to the periaqueductal grey matter.

The Inferior Colliculus. The inferior colliculi (large ovoid structures) formed the caudal portion of the midbrain tectum (online suppl. fig. M-O). The lateral edge of the posterior portion of the midbrain was occupied by the lateral lemniscus (1l), which was seen to directly supply the inferior colliculus with ascending fibres. Within the inferior colliculus, three distinct regions were observed: the dorsal cortex of inferior colliculus (DCIC), the central nucleus of inferior colliculus (CIC), and the external cortex of inferior colliculus (ECIC). No clear layering of the ECIC was evident with any of the stains used in the current study. All three divisions contained parvalbuminimmunopositive neurons and neuropil, and the immunopositive neurons were most densely packed within the CIC. The DCIC also contained calretinin-immunopositive neurons and neuropil, but no calretinin-immunopositive neurons or neuropil were observed within the CIC or ECIC. No calbindin-immunopositive neurons or neuropil were observed in any of the subdivisions of the inferior colliculus. The inferior colliculus was large, attaining a maximum diameter of $10 \mathrm{~mm}$, but did not appear to be disproportionately so when compared to those seen in other mammals, such as microchiropterans and cetaceans. Fibre tracts emerging from the dorsal surface of the inferior colliculus swept from lateral to medial over the dorsal surface and coalesced to form a substantial commissure of the inferior colliculus, which crossed the midline in a position dorsal to the periaqueductal grey matter.

The Magnocellular Division of the Red Nucleus. The mammalian red nucleus is typically composed of magnocellular and parvocellular divisions. In the current examination we could only find conclusive architectonic evidence for the magnocellular division of the red nucleus, as the architectonic appearance of the parvocellular divi-

Elephant Diencephalon and Brainstem sion was not clearly demarcated from the surrounding midbrain tegmentum. The neurons within the magnocellular division of the red nucleus were large in comparison to nearby neurons, intensely stained for Nissl substance, and had a multipolar morphology (fig. 6a) (online suppl. fig. J-L). These neurons were moderately densely packed and this division of the red nucleus had a maximal crosssectional diameter of approximately $5 \mathrm{~mm}$. As in other mammals, the magnocellular division of the red nucleus was located in the mid-ventral portion of the midbrain tegmentum, above the pars compacta of the substantia nigra.

The Periaqueductal Grey Matter and Nucleus Ellipticus. As is typical for mammals, the cerebral aqueduct in the elephant midbrain was surrounded by longitudinal columns of grey matter collectively referred to as the periaqueductal or central grey matter. The stains used in the current study, for the most part, did not reveal specific longitudinal columns in the dorsal and lateral aspects of the periaqueductal grey, but the serotonergic immunostaining revealed the dorsal raphe nuclei in the ventral column (see above), while the Nissl staining revealed a large specialization of the periaqueductal grey matter - the nucleus ellipticus (fig. 7a) - and an unusual region surrounding the lateral and dorsal aspects of the walls of the aqueduct which we refer to here as the potential neurogenic zone (fig. 8). Thus, for the most part, the structure of the periaqueductal grey matter of the elephant is typical of that seen in the vast majority of mammals, but two distinct specializations are worth describing in detail (online suppl. fig. G-P).

Surrounding the lateral and dorsal aspects of the wall of the cerebral aqueduct was a thin lamina, 4-10 cells deep, of small granular shaped cells, with somas slightly larger in size than glia somas (fig. 8). These cells were intensely stained for Nissl substance but were not immunoreactive for any of the antibodies used in the current study. Specific staining for neurogenic markers such as Ki-67, doublecortin and glial fibrillary acidic protein did not label these cells, but the appearance and location of these cells indicated that they are a potential neurogenic site. In addition, at the very lateral aspect of the cerebral aqueduct the thickness of this lamina of cells increased significantly, forming a bulge that invested itself into the surrounding periaqueductal grey matter. This lamina of cells was not only apparent in the specimen we examined but was also observed in a 40-year-old female African elephant and a 48-year-old female Asian elephant, meaning that this lamina of cells is a feature common to the brains of elephants. This same lamina, which we identify as a 


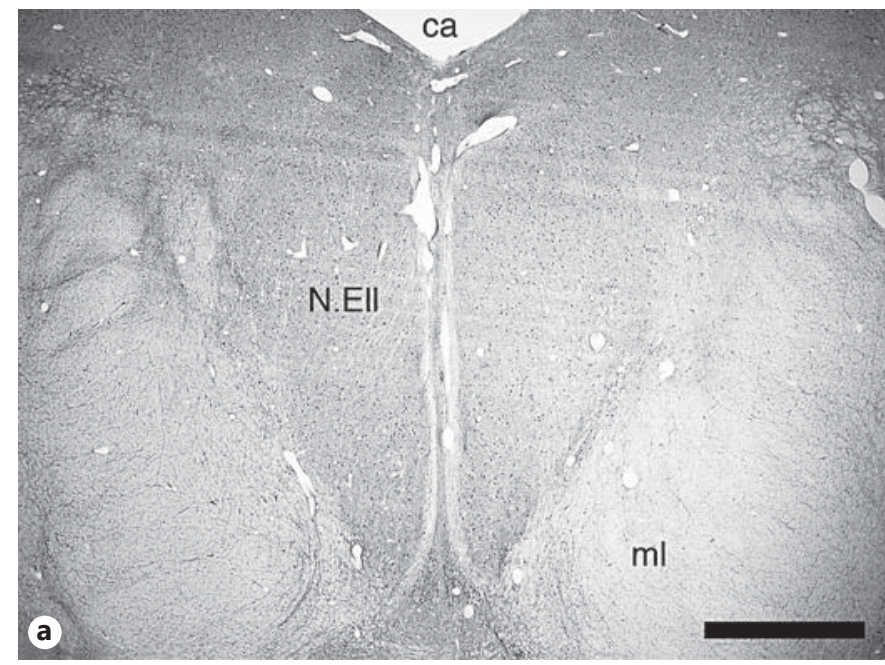

Fig. 7. Lower-power photomicrographs of Nissl-stained sections through the nucleus ellipticus (N.Ell) in the mibrain (a) and the various divisions of the facial nerve nucleus at the pontomedullary junction (b). Note the location of the nucleus ellipticus emerging from the ventrolateral aspect of the periaqueductal grey matter, occupying a position medial to the medial lemniscus ( $\mathrm{ml})$. Scale bar in $\mathbf{a}=4 \mathrm{~mm}$. Note the several subdivisions of the facial nerve

potential neurogenic zone, was found throughout the cerebral aqueduct and extended into the pontine region (see below).

The most outstanding feature of the periaqueductal grey matter in the elephant was the large and specialized nucleus ellipticus, which appeared to be a large ventral expansion of the ventrolateral grey matter column (fig. 7a). This large nucleus had a maximum mediolateral dimension of approximately $8 \mathrm{~mm}$, a dorsoventral dimension of almost $15 \mathrm{~mm}$, and a rostrocaudal dimension of approximately $20 \mathrm{~mm}$. The rostral aspect of this nucleus was located between the caudal-most aspect of the hypothalamus and the medial lemniscus, ventral and lateral to the ventromedial basal nucleus of the dorsal thalamus. Caudally the nucleus was surrounded by thin extensions of the periaqueductal grey matter on the dorsal and lateral aspects of the nucleus before eventually investing itself fully into the ventrolateral column of the periaqueductal grey matter at the level of the oculomotor nucleus, rostral to the serotonergic dorsal raphe complex. Within the nucleus ellipticus the density of neurons was low to moderate and these multipolar neurons often exhibited triangular shaped somas. The only evident immunohistochemical staining within this nucleus with the antibodies used in the current study was neuropil immunopositive for parvalbumin.

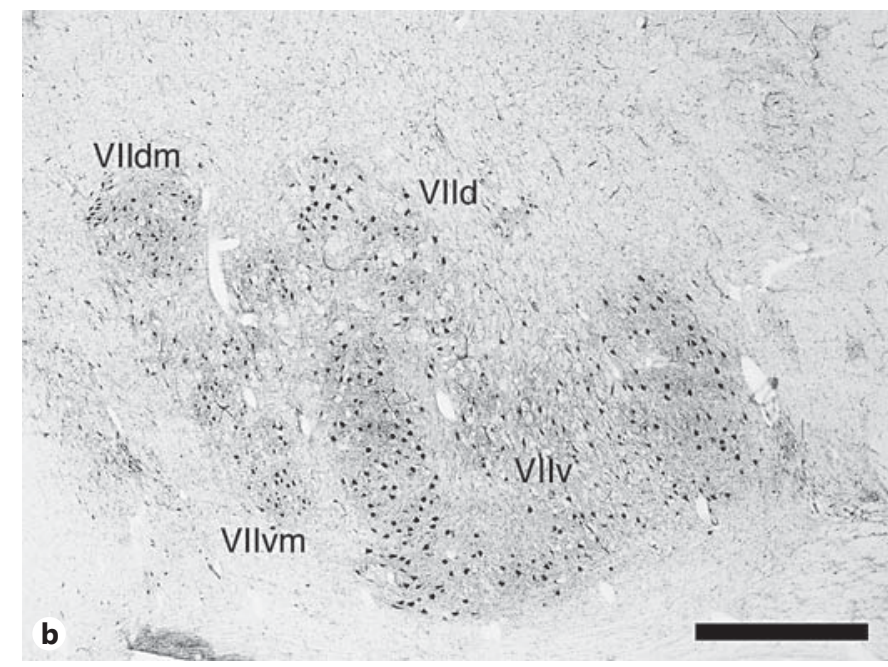

nucleus, including the ventral (VIIv), dorsal (VIId), ventromedial (VIIvm) and dorsomedial (VIIdm) subdivisions. Scale bar in $\mathbf{b}=$ $3 \mathrm{~mm}$. The presence of the nucleus ellipticus and the size and organizational complexity of the facial nerve nucleus appear to be related to vocalization control and control of trunk movement. a, $\mathbf{b}$ Medial is to the left and dorsal to the top. $\mathrm{ca}=$ Cerebral aqueduct.

The Reticular/Tegmental Nuclei of the Midbrain

The reticular formation of the midbrain, which formed the core of this segment of the brainstem, was demarcated rostrally by the ascending medial lemniscus and the descending fasciculus retroflexus and caudally by the decussation of the superior cerebellar peduncle. It was bordered dorsally by the tectum and the periaqueductal grey matter, medially by the midline raphe nuclei, laterally by the presence of the dorsal thalamus, and ventrally by the substantia nigra complex (online suppl. fig. G-P). The midbrain reticular formation had the appearance of a typical mammalian reticular nucleus, with a range of both large and small cells forming a homogeneous mesh-like dendritic network around fascicles of axons. While distinct reticular nuclei, such as the parabrachial, cuneiform and subcuneiform nuclei, are often described in this region of the brain, these were not readily demarcated in the elephant. The neuropil of the entire midbrain reticular formation was immunoreactive for calbindin axons of passage, and moderate densities of calbindin- and calretinin-immunopositive neurons were found scattered throughout this region. Housed within the midbrain reticular formation were the serotonergic median raphe nucleus, the magnocellular division of the red nucleus and the retrorubral catecholaminergic field (see above). There was nothing in the appearance of this region of the 


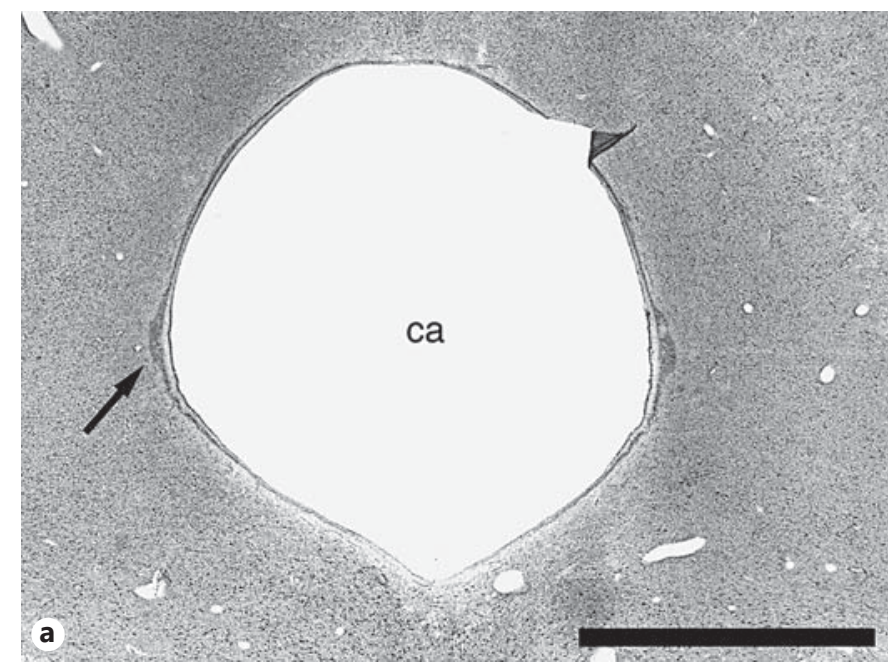

Fig. 8. Photomicrographs of Nissl-stained sections showing the potential neurogenic lining surrounding the walls of the cerebral aqueduct (ca). Note the granular appearance of the cells and the slight bulge of the cells on the lateral aspect of the cerebral aqueduct (arrow). Scale bar $=5 \mathrm{~mm}(\mathbf{a}), 500 \mu \mathrm{m}$ (b) and $250 \mu \mathrm{m}$ (c). Medial is to the left and dorsal to the top of $\mathbf{b}$ and $\mathbf{c}$.

midbrain that differed significantly from that seen in other mammals.

\section{Pons}

The Cranial Nerve Nuclei of the Pons

Within the pons of the African elephant we were able to readily identify three distinct nuclear complexes/individual nuclei associated with specific cranial nerves: the trigeminal nerve and associated nuclei, the abducens nucleus, and the facial nerve and associated nuclei. All of these nuclei/nuclear complexes were located in regions of the pons that can be considered typical of mammals.

The Trigeminal Nerve and Associated Nuclei. The trigeminal nerve was the largest cranial nerve observed for the African elephant brain, with a diameter reaching close to $10 \mathrm{~mm}$ at its largest point. The trigeminal nerve was

Elephant Diencephalon and Brainstem
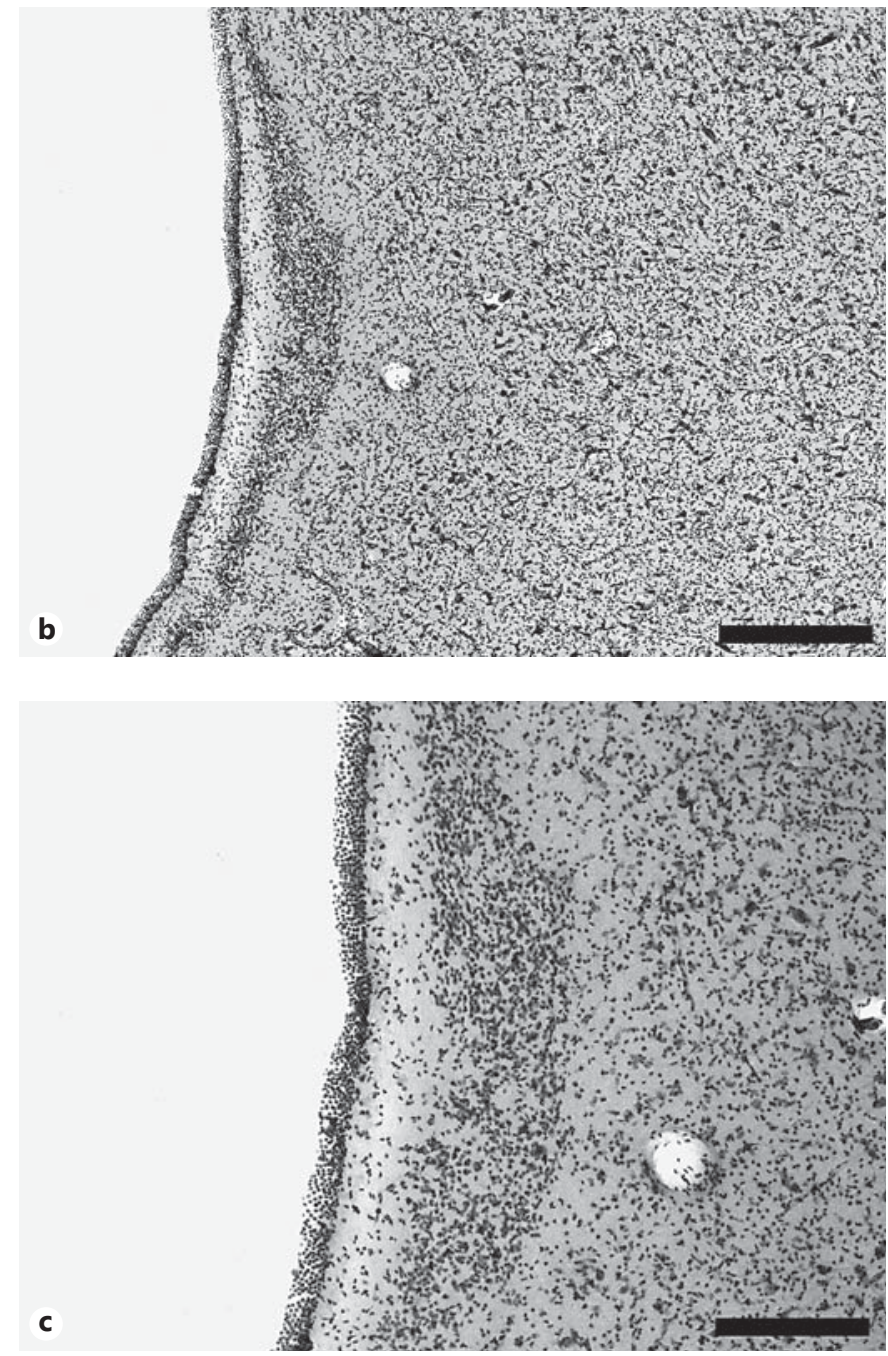

observed to enter/exit the brain via the ventrolateral portion of the pons, investing itself in the middle cerebellar peduncle in a position just lateral to the ventral pontine nucleus (see below) (online suppl. fig. V-Y). The afferent branch of the trigeminal nerve was observed to split into three divisions: one that terminated within the two subdivisions of the principal trigeminal nucleus, one that continued rostrally to form the trigeminal mesencephalic tract, and one that turned and progressed caudally to form the spinal trigeminal tract. The efferent branch was observed to arise from the motor division of the trigeminal nucleus and course dorsal to the principal trigeminal nucleus to join the main trunk of the trigeminal nerve in the middle cerebellar peduncle.

The Principal Trigeminal Nucleus. The principal trigeminal nucleus (PrV) was located medial to the spinal 
trigeminal tract and lateral to the parvocellular column of the pontine reticular formation and the motor division of the trigeminal nerve, from the rostral-most level of the pons, at the same level as the motor trigeminal nucleus, extending caudally to the caudal-most level of the pons, at the same level as the descending arm of the facial nerve (online suppl. fig. U-Y). While for the majority of the pons the dorsomedial division of the $\operatorname{PrV}(\mathrm{PrVdm})$ is the most clearly recognizable trigeminal sensory nucleus, there appears to be a distinct ventrolateral division of this nucleus (PrVvl), as seen in the laboratory rat [Paxinos et al., 2009]. At the caudal end of the pons, $\operatorname{PrV}$ transitions into the medullary portions of the trigeminal sensory nuclei (see below). Both subdivisions of the PrV are invested with neuropil immunopositive to parvalbumin, calbindin and calretinin, with the PrVdm containing both calbindinand calretinin-immunopositive neurons and the PrVvl containing only calbindin-immunopositive neurons.

The Pontine Portion of the Trigeminal Mesencephalic Nucleus (mesV) and Tract (mesVtr). The ganglion cells that form this nucleus in the pons present as a continuation of the low-density cluster of cells that form this nucleus in the midbrain. Again, they are located on the lateral-most portion of the periventricular grey matter at this level of the brainstem, but this nucleus finishes at the rostral-most level of the locus coeruleus and is thus not represented throughout the entire length of the pons (online suppl. fig. P-T). From the trigeminal nerve, a cluster of axons passes forward and dorsally to form the trigeminal mesencephalic tract (mesVtr) located on the lateral border of the periventricular grey matter from the caudalmost level of the locus coeruleus.

The Trigeminal Motor Nucleus. A moderately dense cluster of large ChAT-immunopositive neurons, with the appearance of typical multipolar motor neurons, was designated as the trigeminal motor nucleus (online suppl. fig. $\mathrm{V}-\mathrm{Y}$ ). This nucleus was located in the dorsal lateral part of the parvocellular reticular pontine region, in a position just rostral and medial to the descending arm of the facial nerve. As mentioned above, the efferent axonal bundles from these neurons coalesce to form a distinct ChATimmunopositive fasciculus that traverses over the PrVdm to join the large afferent branch of the trigeminal nerve and exits from the ventrolateral portion of the middle cerebellar peduncle.

The Abducens Nerve and Nucleus. A densely packed cluster of large ChAT-immunopositive neurons, with the typical multipolar morphology of motor neurons, was located in the dorsomedial aspect of the parvocellular reticular pontine region, nestled beneath the genu of the facial nerve (online suppl. fig. Z, AA). This cluster of neurons was designated as the abducens nucleus. No distinct efferent fibre bundles were observed in our preparation; however, a previous report indicates that the abducens nerve exits the brainstem at the pontomedullary junction as seen in other mammals [Shoshani et al., 2006].

The Facial Nerve and Facial Nuclear Complex. The facial nerve emerged from the dorsal aspect of the facial nuclear complex, travelled dorsally to the roof of the caudal aspect of the pons, bent laterally over the abducens nucleus to form the facial colliculus, and then extended ventrally to exit at the pontomedullary junction ventral to the vestibulocochlear nerve - a path typical of mammals. This nerve is, in a relative sense, quite large and can measure up to $3 \mathrm{~mm}$ in diameter at its largest point, although Shoshani et al. [2006] recorded slightly larger diameters $(3.3-4 \mathrm{~mm})$ for this nerve. The large size of this nerve is also reflected in the large size and parcellation of the facial nerve nuclear complex (online suppl. fig. X-FF). Using ChAT immunohistochemistry combined with Nissl staining we could determine that the facial nerve nuclear complex consists of five distinct nuclei found in the ventral and caudal aspect of the parvocellular column of the pontine reticular formation. Distinct dorsal (VIId), ventral (VIIv), dorsomedial (VIIdm) and ventromedial (VIIvm) nuclei were evident, and the largest of these was the ventral nucleus (fig. 7b). The neurons within each of these nuclei were all ChAT immunopositive and exhibited the typical motor neuron multipolar morphology. A cluster of smaller, but medium sized, ChAT-immunopositive neurons was observed in the more caudal aspects of the facial nuclear complex in a position above the VIIv nucleus and lateral to the VIId nucleus. These medium sized neurons were less densely packed than in the four previously mentioned nuclei and were assigned to the parasympathetic nuclear component of the facial nucleus (pVII), also referred to as the superior salivatory nucleus.

The Ascending and Descending Fibre Pathways of the Pons

The pons of the African elephant is notable for several large fibre pathways which are contained within this portion of the brainstem. The rostral border of the pons is clearly demarcated by the decussation of the superior cerebellar peduncle (scp, also known as the brachium conjunctivum). From the decussation, the fibres coalesce laterally to form the distinctive arc of the scp, in a position dorsolateral to the pontine parvocellular reticular nucleus (PCRt) (online suppl. fig. P-AA). The scp continues caudally in this position until the level of the trigeminal mo- 
tor nucleus (Vmot), where it shifts dorsally to invest into the white matter of the cerebellum. The extremely large middle cerebellar peduncle ( $\mathrm{mcp}$ ) is seen to arise from the lateral aspect of the ventral pontine nucleus (VPO, see below) (online suppl. fig. P-CC). The mcp of the African elephant attains a diameter of approximately $20 \mathrm{~mm}$ at its greatest breadth, before progressing laterally to invest into the white matter of the cerebellum. The three cerebellar peduncles (see below for a description of the inferior cerebellar peduncle) appear to follow the pattern typically seen in mammals, but in the elephant all three peduncles seem to be relatively enlarged when compared to other mammalian species.

Within the pons a distinct medial column of white matter lies between the raphe reticular column and the gigantocellular reticular nucleus (see below). This column extends from the dorsal aspect of the pontine midline, through to the ventral-most aspect, and is expanded significantly in a lateral direction in the ventral portion of this column, extending around the lateral aspect of the pons. This medial white matter column was observed to run the entire rostrocaudal extent of the pons and housed several distinctive pathways, such as the medial longitudinal fasciculus, the lateral lemniscus, the medial lemniscus, and the longitudinal fasciculus of the pons, and several less distinct pathways, such as the tectospinal tract (online suppl. fig. P-AA). The mediodorsal aspect of this white matter column was occupied by the medial longitudinal fasciculus (mlf), which attained a maximum diameter of approximately $1 \mathrm{~mm}$. Below the mlf the medial white matter column appears to be a conglomeration of several pathways, including the tectospinal pathway, but these could not be individually identified in our material. At the ventromedial aspect of the medial white matter column, the large longitudinal fasciculus of the pons (lfp) was readily identified as a caudal continuation of the cerebral peduncles of the midbrain (see above). This large fasciculus lies immediately dorsal to the ventral pontine nucleus (see below) and runs the entire rostrocaudal extent of the pons, where it continues as the distinct pyramidal tracts seen in the medulla oblongata (see below). The medial lemniscus was observed to surround the dorsal, medial and lateral aspects of the lfp. The medial lemniscus formed a consolidated mass lateral to the lfp in the rostral portion of the pons and was observed to move dorsally and laterally around the pontine reticular formation before entering the midbrain (see above). A distinct lateral lemniscus was observed to coalesce around the lateral margin of the lfp and medial lemniscus in the rostral portions of the pons. The lateral lemniscus was located on the very lateral margin of the pons, lateral to the superior cerebellar peduncle, and was oriented on a dorsoventral plane before continuing into the midbrain (see above). The lateral lemniscus formed the lateral-most distinct fibre pathway of the pons. A small, reticulated, nucleus of the lateral lemniscus was evident.

\section{The Neuromodulatory Nuclei of the Pons}

The Cholinergic Nuclei of the Pons. Neurons immunoreactive to the choline acetyltransferase antibody $(\mathrm{ChAT}+)$ were used to delineate the parabigeminal nucleus, as well as the pedunculopontine (PPT) and laterodorsal tegmental (LDT) nuclei within the pons (online suppl. fig. $\mathrm{N}-\mathrm{R}$ ). The parabigeminal nucleus, a relatively small nucleus in the elephant, was located at the very lateral margin of the rostral-most pontine tegmentum in a location ventrolateral to the inferior colliculus and lateral to the lateral lemniscus. Within this nucleus the ChAT+ neurons were moderately densely packed. The ChAT + neurons forming the PPT were located through the entire rostral portion of the PCRt, abutting the medial aspect of the superior cerebellar peduncle, with some neurons extending around this peduncle into the parabrachial region. The neurons forming the PPT appear to be more widespread than seen in many other mammals and also appear to have a higher relative density than seen in other mammals [Dell et al., 2010]. Within the periventricular grey matter, caudal and lateral to the oculomotor nucleus, a moderate to high density of ChAT+ neurons was designated as those forming the LDT nucleus. The ventrolateral border of the LDT neurons was contiguous with the dorsomedial border of the PPT nucleus, the only reason to separate these two nuclei being the transition from the periventricular grey matter to the pontine tegmentum. The neurons of the LDT were found from the level of the trochlear nucleus, extending approximately $15 \mathrm{~mm}$ caudal into the pons. For all three nuclei, the position appears typical of that seen in other mammals, although the PBg does appear to be in a location slightly rostral to that typically seen.

The Catecholaminergic Nuclei of the Pons: The Locus Coeruleus Complex. Within the pontine region a large number of tyrosine hydroxylase-immunopositive (TH+) neurons forming the locus coeruleus complex were readily observed from the rostral-most to the caudal-most level of the pons (online suppl. fig. O-CC). The locus coeruleus complex could be readily subdivided into five nuclei, these being: the subcoeruleus compact portion (A7sc), the subcoeruleus diffuse portion (A7d), the locus coeruleus diffuse portion (A6d), the locus coeruleus medial portion (A6m) and the fifth arcuate nucleus (A5). We did 


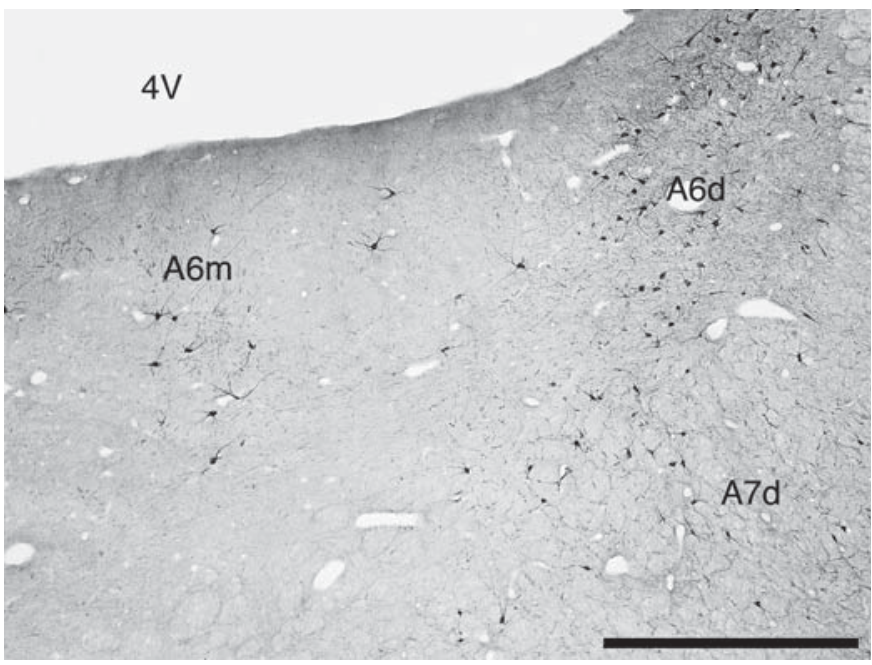

Fig. 9. Low-power photomicrograph of the locus coeruleus region showing the diffuse division of the locus coeruleus (A6d), the novel medial division of the locus coeruleus $(\mathrm{A} 6 \mathrm{~m})$ and the diffuse division of the subcoeruleus (A7d) with neurons immunopositive for tyrosine hydroxylase. Scale bar $=2 \mathrm{~mm}$. Medial is to the left and dorsal to the top. $4 \mathrm{~V}=$ Fourth ventricle.

not identify the dorsolateral division of locus coeruleus (A4), likely due to the small size of this nucleus and the location of it within the medial portion of the cerebellar peduncles [e.g. Kruger et al., 2012] that were not included in the block of tissue for sectioning. Within the dorsal portion of the parvocellular pontine tegmentum (PCRt, see below) adjacent to the ventrolateral region of the periventricular grey matter (see below), a tightly packed cluster of $\mathrm{TH}+$ neurons represented the $\mathrm{A} 7$ compact portion of the LC. This division is the same as what was previously described as the subcoeruleus [Dahlström and Fuxe, 1964; Olson and Fuxe, 1972]. Ventral and lateral to the $\mathrm{A} 7 \mathrm{sc}$, a diffusely organized aggregation of $\mathrm{TH}+$ neurons formed the A7d nuclear complex. These neurons were located throughout the vast majority of the PCRt mostly in a position rostral to the trigeminal motor nucleus (Vmot) and in places were found lateral to the superior cerebellar peduncle in the lateral parabrachial region. Within the lateral portion of the periventricular grey matter a moderate density of $\mathrm{TH}+$ neurons was assigned to the A6d nucleus. The neurons of this group were found adjacent to the lateral border of the periventricular grey matter and were not observed to continue across the grey matter to the wall of the ventricle as seen in Murid rodents [Dahlström and Fuxe, 1964; Kruger et al., 2012]; rather they were observed to be a moderately loosely packed cluster of neurons located in the ventrolateral half of the periventricular grey matter as seen in many other mammalian species [e.g. Dell et al., 2010; Kruger et al., 2010]. A novel finding in the African elephant periventricular grey matter was a cluster of $\mathrm{TH}+$ neurons located in a topographically distinct location medial to the A6d within the periventricular grey matter. We have termed this small cluster of neurons the $\mathrm{A} 6 \mathrm{~m}$, to acknowledge both the location of these neurons and the morphological similarity to the neurons of the A6d (fig. 9). In the ventrolateral pontine tegmentum, from the level of Vmot to the caudal-most level of the pons, in a position amongst the subdivisions of the superior olivary nucleus and ventrolateral and caudal to $\mathrm{A} 7 \mathrm{~d}$, a small cluster of $\mathrm{TH}+$ neurons formed the A5 nucleus. These neurons formed a mesh-like dendritic network around the ascending fascicles located within the ventrolateral PCRt.

The Serotonergic Nuclei of the Pons. In the very rostral portion of the pons, two distinct populations of serotonergic immunoreactive neurons were observed, forming the dorsal raphe caudal (DRc) and median raphe (MnR) nuclei (online suppl. fig. O-R). Both of these nuclei are caudal extensions of the rostral cluster of serotonergic nuclei found in the midbrain (see above). The DRc appears to be a caudal extension of the DRl (see above) and is classified as such on the basis of the similarity of the neuronal morphology (large multipolar serotonergic immunoreactive neurons) and topographic continuity of the cell group. The neurons of the DRc were observed within the dorsomedial half of the periventricular grey matter of the pons, spreading from medial to lateral across the grey matter. We have classified this cluster of serotonergic neurons as an independent nucleus due to the lack of serotonergic immunoreactive neurons in this region in the brain of monotremes [Manger et al., 2002; Maseko et al., 2007; Dell et al., 2010]. The median raphe nucleus (MnR) was characterized by two distinct, densely packed $5 \mathrm{HT}+$ neuronal columns located on either side of the midline in a para-raphe position, at the very rostral aspects of the pontine midline. This pontine extension of the MnR is sometimes referred to as the pontine raphe nucleus [Dahlström and Fuxe, 1964; Paxinos et al., 2009]; however, it is evident that these two columns of serotonergic neurons are a caudal extension of the main cluster of neurons forming this nucleus in the midbrain (see above) and do not form a distinct pontine serotonergic nuclei.

\section{The Intrinsic Nuclei of the Pons}

The Periventricular Grey Matter. In the dorsal part of the pons, adjacent to the floor of the fourth ventricle, a 
region of grey matter that is a caudal extension of the periaqueductal grey matter (see above) is referred to as the pontine periventricular grey matter due to the opening of the cerebral aqueduct into the fourth ventricle (online suppl. fig. O-Z). This periventricular grey matter area occupies this location along the entire rostrocaudal extent of the pons and merges with the rostral portion of grey matter of the tractus solitarius at the pontomedullary junction. At the rostral limits of the pons, the periventricular grey matter has a depth of approximately $4 \mathrm{~mm}$, but this depth thins as it extends caudally to approximately $1 \mathrm{~mm}$ thick or less at the level of the trigeminal motor nucleus. A distinct nucleus located in the rostral medial portion of the periventricular grey matter, and invested with a high density of calbindin-immunoreactive neurons, appears to represent the posterodorsal tegmental nucleus (PDTg). The remainder of this grey matter was homogenous in appearance, but the range of immunostains used in the current study revealed the presence of the cholinergic laterodorsal tegmental nucleus, the catecholaminergic medial and diffuse divisions of the locus coeruleus, and the serotonergic caudal division of the dorsal raphe (see above) within differing portions of this grey matter. Calbindin- and calretinin-immunopositive neurons were also scattered throughout this grey matter, but their distribution did not afford parcellation of this region.

The Potential Neurogenic Zone of the Pons. A thin layer of cells occupying the dorsal aspect of the pontine periventricular grey matter, in direct apposition to the fourth ventricle, appeared to be a continuation of the potential neurogenic zone observed in the midbrain (online suppl. fig. $\mathrm{O}-\mathrm{Z}$ ). In the rostral portions of the pons, this thin lamina of cells was located a few millimetres away from the midline, but more caudally in the pons it was observed to merge across the midline. This layer of cells was observed throughout the entire rostrocaudal extent of the pons and was seen to merge with a similar region in the dorsal aspect of the medulla oblongata (see below). Staining for neurogenic markers such as Ki-67, doublecortin and glial fibrillary acidic protein did not label these cells. Despite this, the appearance and location of this layer of cells indicate that it is a potential neurogenic site.

The Superior Olivary Complex. The superior olivary nuclear complex was located in the ventral portion of the parvocellular reticular nucleus (PCRt), in a position rostral to the facial nerve nucleus (online suppl. fig. $U-Z$ ). The largest nuclear subdivision of this complex was the lateral superior olive (LSO), which at certain levels of section was subdivided into several distinct parts related to the afferent and efferent pathways associated with this nucleus and the passage of the descending arm of the facial nerve. The LSO had mediolateral dimensions of approximately $5 \mathrm{~mm}$ and spanned a rostrocaudal distance of approximately $20 \mathrm{~mm}$. The remaining components of the superior olive were substantially smaller in size and included the medial superior olive (MSO), the medioventral periolivary nucleus (MVPO), and the superior paraolivary nucleus (SPO). All of the subdivisions within this complex were invested with parvalbumin-, calbindin- and calretinin-immunoreactive neuropil and a moderate density of calbindin-immunopositive neurons. The LSO also exhibited neurons immunoreactive for calretinin.

The Ventral Pontine Nucleus. At the base of the pons, occupying the ventral aspect of the entire rostrocaudal aspect of the pons, the large ventral pontine nuclei were readily observed (online suppl. fig. N-CC). The appearance of the neurons that formed this nucleus was typical of those found in mammals, and these were not immunoreactive for any of the antibodies used in the current study. The neurons within this nucleus appear to be arranged in horizontally oriented sheets, in between which transverse (corticobulbar, corticospinal and corticopontine) and longitudinal (pontocerebellar) fibre bundles were observed. From the lateral aspect of the ventral pontine nucleus the large middle cerebellar peduncle emerged.

\section{The Reticular/Tegmental Nuclei of the Pons}

The reticular formation of the pons, forming the core of this segment of the brainstem, was demarcated rostrally by the decussation of the superior cerebellar peduncle and caudally by the descending limb of the facial nerve (online suppl. fig. R-BB). As in other mammals, the pontine reticular formation can be subdivided into three major rostrocaudally oriented cell columns: (1) the raphe column, (2) the gigantocellular/magnocellular column (GiCRt), and (3) the parvocellular column (PCRt). The raphe column consisted of two parasagittal cell columns, often only a few cells wide, located along the entire rostrocaudal extent of the pons. The most ventral portion of this raphe column was seen to expand laterally, forming the distinct reticular tegmental nucleus of the pons (RtTg) along much of the rostrocaudal length of the pons. At some levels of the pons the RtTg was seen to extend over the longitudinal fasciculus of the pons forming a very laterally located subdivision. A thin (less than $1 \mathrm{~mm}$ ), vertically oriented fibre sheet divided the raphe columns at the midline. In the very rostral parts of the pontine raphe the cells forming the raphe columns were immunopositive 
for serotonin (the median raphe nucleus, see above). The remaining neurons of these columns did not exhibit any specific immunoreactivity to the antibodies used in the current study, and thus we could not subdivide this column into more distinct components. At most levels of the pons the raphe column, and the ventral expansion (RtTg), was isolated from the laterally located GiCRt by a column of white matter that varied in thickness (incorporating the medial longitudinal fasciculus, medial lemniscus and longitudinal fasciculus of the pons, see above).

The gigantocellular reticular nucleus (GiCRt) was found lateral to the central fibre column, extending the entire rostrocaudal length of the pons. The GiCRt had the appearance of a typical mammalian reticular nucleus, with the large cells forming a homogeneous mesh-like dendritic network around fascicles of descending axons. At the lateral margin of the caudal portion of the GiCRt, close to its border with the PCRt, a column of tyrosinehydroxylase-immunopositive neurons, the rostral ventrolateral tegmental group ( $\mathrm{C} 1$, see below), was located. Occasional calbindin- and calretinin-immunopositive neurons were observed in the GiCRt, and the axons passing through this nucleus were often immunopositive for these two markers.

The parvocellular reticular nucleus (PCRt) was found lateral to the GiCRt at all levels throughout the pons and was bordered laterally by the superior cerebellar peduncle, the principal trigeminal nucleus and the descending arm of the facial nerve. The marked reduction in size of the soma forming this nucleus provided a reliable delineation of the medial edge of this nucleus at its border with the GiCRt. Again, the typical mammalian reticular appearance was present, and occasional calbindin- and calretinin-immunopositive neurons were observed. The axons passing through this nucleus were also immunopositive for these two markers. The region of the PCRt rostral to the trigeminal motor nucleus (see above) housed the cholinergic pedunculopontine tegmental nucleus (PPTg) and the catecholaminergic subcoeruleus compact and diffuse nuclei (A7sc and A7d). Caudal to this level, in the ventral portion of the PCRt, the catecholaminergic fifth arcuate nucleus (A5, see above) was located.

\section{Medulla Oblongata}

The Cranial Nerve Nuclei of the Medulla Oblongata

Within the medulla oblongata of the African elephant, we were able to readily identify six distinct nuclear complexes/individual nuclei associated with specific cranial nerves: the spinal trigeminal tract and associated nuclei, the vestibulocochlear nerve and associated nuclei, the nu- cleus ambiguus, the inferior salivatory nucleus, the dorsal motor vagal nucleus, and the hypoglossal nucleus. All of these nuclei/nuclear complexes were located in regions of the medulla oblongata that can be considered typical of mammals.

The Spinal Trigeminal Tract (Sp5) and Associated Nuclei. The spinal trigeminal tract (Sp5) and associated nuclei were found lateral to the medullary tegmentum/reticular formation, along the entire rostrocaudal extent of the medulla, crossed the spinomedullary junction and merged with the dorsal horn of the cervical spinal cord (online suppl. fig. Y-XX). At the rostral level of the medulla, these structures were bordered laterally by the inferior cerebellar peduncle and some other smaller tracts, whereas caudally in the medulla they formed the lateral border of the brain. The spinal trigeminal tract itself arises in the pons and is formed by the caudal branch of the main trigeminal afferent nerve. The tract is consistently located on the lateral aspects of the trigeminal sensory nuclei observed in the medulla. The Sp5 itself is consistently found in the lateral and laterodorsal aspects of the spinal trigeminal sensory nuclei.

At the pontomedullary junction, the dorsomedial division of the principal trigeminal nucleus ( $\mathrm{PrVdm}$ ) in the pons is seen to transition to the oral subdivision of the spinal trigeminal nucleus (Sp5o). The Sp5o nucleus exhibits parvalbumin, calbindin- and calretinin-immunopositive neuropil and a moderate density of calretinin-immunopositive cells. In the PrVdm there is a high density of calretinin-immunopositive cells; thus, this transition was marked by a noticeable decrease in the density of calretinin-immunopositive cells. Within 10 $\mathrm{mm}$ caudal of this transition, the $\mathrm{Sp} 5 \mathrm{o}$ is seen to transition into the lateral subdivision of the spinal trigeminal nucleus (Sp5l). The transition from Sp5o to Sp5l is marked by the absence of parvalbumin-immunopositive neuropil, the presence of the occasional parvalbumin-immunopositive cell, and the presence of a low density of calbindinimmunopositive cells in Sp51. This transition occurs close to the caudal level of the facial nerve nuclear complex. A short distance caudal to this transition, at the level of the rostral pole of nucleus ambiguus, the dorsal division of the dorsomedial spinal trigeminal nucleus (DMSp5D) appeared in a location dorsal to the Sp5l. The DMSp5D is characterized by the presence of calbindin-immunopositive cells and neuropil and calretinin-immunopositive neuropil. The DMSp5D transitions into the ventral division of the dorsomedial spinal trigeminal nucleus (DMSp5V), which is marked by the absence of any parvalbumin or calbindin immunoreactivity and the presence 
of a low density of calretinin-immunoreactive neurons. The dorsal and ventral subdivisions of the DMSp5 of the elephant were not topologically arranged as described in the laboratory rat [Paxinos et al., 2009]; rather than being in a position that indicates that one lies dorsal to the other as in the rat, it appears that these nuclei are positioned rostral/caudal to each other.

At the level of the tractus solitarius the Sp5l nucleus is seen to transition into the caudal subdivision of the spinal trigeminal nucleus (Sp5c). The Sp5l nucleus becomes thinner and is replaced medially by the $\mathrm{Sp} 5 \mathrm{c}$. This transition is marked by the absence of any neuropil immunoreactivity for parvalbumin, calbindin or calretinin, the absence of any parvalbumin-immunopositive neurons, and the presence of moderate densities of both calbindinand calretinin-immunopositive neurons. As the Sp5l tapers away, the gelatinous layer of the caudal spinal trigeminal nucleus $(\mathrm{Ge} 5)$ appears as a reticulated nucleus within the main fibre bundle of the spinal trigeminal tract, investing into the tracts as small islands from the medial side of the tract. Both calbindin- and calretininimmunopositive neurons are present within the Ge5. This nucleus enlarges caudally and becomes a solid nuclear mass interposed between the Sp $5 \mathrm{c}$ and the Sp5 at the level of the decussation of the pyramidal tracts. As the medulla transitions into the spinal cord, the Ge5 splits into distinct inner (Ge5i) and outer (Ge5o) sublamina that eventually become the first (1, Ge5o) and second (2, $\mathrm{Ge} 5 \mathrm{i}$ ) layers of the dorsal horn of the cervical spinal cord. The neurochemical identity of these cervical spinal cord layers appears identical to that of the two sublamina of the Ge5. In a similar manner, the $S p 5 c$ nucleus tapers and flattens to transition into the third (3) layer of the dorsal horn of the cervical spinal cord, again maintaining a similar neurochemical identity.

The Vestibulocochlear Nerve and Cochlear and Vestibular Nuclei. The vestibulocochlear nerve enters the lateral aspect of the medulla at the same level as the facial nerve exits. It is a moderately large nerve, approximately $2.5 \mathrm{~mm}$ in diameter prior to investing into the medulla. As it enters the medulla the nerve spreads to provide innervation to the cochlear nuclei and the vestibular nuclei. The cochlear portion of the nerve invests directly into the cochlear nuclei that are located on the lateral and laterodorsal aspects of the rostral medulla oblongata. The vestibular branch of this nerve passes ventral to the inferior cerebellar peduncle and laterodorsal to the spinal trigeminal tract to supply the vestibular nuclear complex.

Elephant Diencephalon and Brainstem
The Cochlear Nuclei. The cochlear nuclear complex was located in the dorsal lateral surface of the rostral medulla, lateral to the inferior cerebellar peduncle (online suppl. fig. CC, DD). We could readily identify the dorsal cochlear nucleus, the granule cell layer of the cochlear nucleus, and the ventral cochlear nucleus. Within the dorsal cochlear nucleus, both parvalbumin and calretinin-immunopositive neurons were found. While the calretinin-immunopositive neurons were found throughout the depth of the nucleus, the parvalbumin-immunopositive neurons appeared restricted to the upper half of the nucleus in quite a high density. This arrangement of dense dorsally located parvalbumin-immunopositive neurons indicates that the dorsal cochlear nucleus in the African elephant has two distinct laminas. The granular cells within the cochlear nuclear complex were observed to form a distinct layer that bridged the gap between the dorsal and ventral cochlear nuclei. This granule cell layer was readily identified due to the small, densely packed granular cells located within the region. We could not readily divide the ventral cochlear nucleus into anterior and posterior parts based on the stains employed in the current study. The ventral cochlear nucleus was the largest of the three nuclei and was located on the very lateral aspect of the medulla in its rostral part and was identified between the inferior and middle cerebellar peduncles. The caudal pole of this nucleus appeared to be segregated into three parts due to the investment of fibre pathways entering the nucleus, but the appearance of these three parts did not warrant assigning them to distinct nuclei.

The Vestibular Nuclei. The vestibular nuclear complex was located in the dorsal aspect of the rostral medulla in a position dorsal to the spinal trigeminal system, and medial to the inferior cerebellar peduncle (online suppl. fig. $\mathrm{Z}-\mathrm{EE}$ ). While there appear to be several nuclei within the vestibular nuclear complex, our staining only allowed the confident identification of the lateral vestibular nucleus (LVe) due to the presence of large, multipolar neurons that were intensely stained with cresyl violet. In the region dorsal to the LVe, a cluster of neurons that likely represented both the superior vestibular nucleus and the vestibulocerebellar nucleus ( $\mathrm{VeCb}$ ) was seen to invest into the superior cerebellar peduncle. Medial to the LVe, extending close to the floor of the fourth ventricle, was a significant region of grey matter that likely represents the medial vestibular nucleus (MVe), although the homogenous architectural appearance did not allow for identification of any specific subdivisions in this region.

The Nucleus Ambiguus (N.Amb). A cluster of large, ChAT-immunopositive neurons, exhibiting the typical 
structure of multipolar motor neurons, was observed at the border of gigantocellular and parvocellular reticular columns of the medullary tegmentum, within the upper portion of the ventral half of the tegmentum (online suppl. fig. FF-KK). These neurons were assigned to the nucleus ambiguus. The nucleus ambiguus was observed to extend from the level of the facial nerve nuclear complex to the level of the area postrema, covering a rostrocaudal distance of approximately $20 \mathrm{~mm}$. Although we could not follow the efferent axonal bundle from this nucleus, it would be fair to assume that, as in other mammals, it supplies both the vagal and the glossopharyngeal nerves with efferent motor fibres.

The Preganglionic Motor Neurons of the Inferior Salivatory Nucleus ( $p I X)$. A small cluster of medium sized ChAT-immunopositive motor neurons, located in the very rostral aspect of the grey matter of the tractus solitarius, rostral to the rostral pole of the dorsal motor vagal nucleus, were assigned to the inferior salivatory nucleus (online suppl. fig. FF). The ChAT immunoreactivity, medium size and multipolar morphology typical of preganglionic motor neurons indicated this affiliation.

The Dorsal Motor Vagal Nucleus (X). A discreet, densely packed nucleus of ChAT-immunopositive, mediumsized neurons located in the lateral aspect of the periventricular grey matter of the dorsal medulla oblongata was assigned to the dorsal motor vagal nucleus (online suppl. fig. GG-XX). This nucleus was observed to extend from the level of the facial nerve nuclear complex to the level of the decussation of the pyramidal tract at the spinomedullary junction. In the caudal medulla, it was found in a position approximately $3 \mathrm{~mm}$ lateral to the central canal, whereas more rostrally it was invested within the grey matter of the solitary tract. The cell bodies have the typical appearance of motor neurons, but are smaller in size, indicating their non-somatic, parasympathetic nature. While this nucleus has a diameter of approximately $2 \mathrm{~mm}$ across on the coronal plane, it extends slightly more than $50 \mathrm{~mm}$ on the rostrocaudal axis, making it quite a large nucleus. Again, we could not follow the efferent fibre bundles from this nucleus, but it would be fair to assume that, as in other mammals, it supplies the vagal nerve with parasympathetic efferent fibres. A small number of ChAT-immunopositive neurons appeared to extend caudally from this nucleus and spread laterally along the border of laminas 5 and 7 of the grey matter of the spinal cord, forming a distinct caudal continuation of the nucleus into the upper cervical levels of the spinal cord.

The Hypoglossal Nucleus (XII). The hypoglossal nucleus was readily defined as a dense cluster of ChAT-immu- nopositive neurons located in the periventricular grey matter of the dorsal medial aspect of the caudal medulla oblongata (online suppl. fig. II-PP). The cell bodies exhibited the typical large multipolar motor neuron appearance, being larger than those seen in the nearby dorsal motor vagal nucleus $(\mathrm{X})$. The hypoglossal nucleus appears at a level slightly caudal (about $5 \mathrm{~mm}$ ) to the rostral pole of the dorsal motor vagal nucleus and ends approximately $20 \mathrm{~mm}$ rostral of the caudal pole of the dorsal motor vagal nucleus. Thus, it has a rostrocaudal extent of approximately $25 \mathrm{~mm}$ but reaches a maximum diameter of almost $4 \mathrm{~mm}$ in the rostral portions of the nucleus. The hypoglossal nerve was observed to emerge from the ventral portion of the nucleus, especially in the more rostral regions, and took a course lateral to the inferior olivary nuclear complex to emerge from the ventral surface of the medulla oblongata in a position lateral to the bulge denoting the inferior olivary complex.

The Ascending and Descending Fibre Pathways of the Medulla Oblongata

Within the medulla there are two major regions of distinct white matter: a medial column and a lateral sheath. The largest region of white matter, the medial column, lies between the raphe reticular column and the gigantocellular reticular nucleus (see below). This column extends from the dorsal-most aspect of the medulla, through to the ventral-most aspect, and at its most lateral margin can extend up to $5 \mathrm{~mm}$ from the midline. This medial column was observed to run the entire rostrocaudal extent of the medulla and houses several distinctive pathways, such as the medial longitudinal fasciculus and the medial lemniscus and the pyramidal tract, and several less distinct pathways, such as the tectospinal tract. The lateral sheath of white matter is seen to course around the ventral and lateral edges of the medulla and is formed of pathways such as the ventral, rostral and dorsal spinocerebellar, cuneocerebellar and olivocerebellar, most of which ultimately coalesce into a large inferior cerebellar peduncle in the more dorsal and rostral portions of the medulla.

The Medial White Matter Column of the Medulla Oblongata. As mentioned above, a large white matter column was found throughout the medial aspect of the medulla (online suppl. fig. Z-XX). The only location where the extent of this white matter column was diminished was at the levels where the inferior olivary nuclear complex was present. At these levels, the column was split above and below this complex. In the very dorsal part of this white matter column, a distinct medial longitudinal fasciculus (mlf) could be identified. In the rostral medul- 
la the mlf could be readily distinguished from the remainder of the white matter column, but the distinction of this fasciculus from the remainder of the white matter became less distinct in the caudal medulla. Toward the spinomedullary junction, the medial longitudinal fasciculus was seen to merge with the ventral funiculus of the spinal cord.

In the ventral-most portion of the medial white matter column a distinct pyramidal tract was observed to run the entire length of the medulla. The pyramidal tract was seen to form as a continuation of the longitudinal fasciculus of the pons, forming a distinct, horizontally flattened pair of fibre pathways, almost $5 \mathrm{~mm}$ in width, on either side of the midline in the most ventral part of the medulla. Further caudally in the medulla, the horizontally flattened shape of the pyramidal tracts was seen to expand in the dorsoventral plane, with a shortening of the mediolateral dimension. The tract maintained this position and shape until the very caudal parts of the medulla, where it was observed to expand dorsally and decussate (online suppl. fig. BB-XX). The decussation extends for approximately $30 \mathrm{~mm}$ on the rostrocaudal plane, with the pyramidal tract occupying the ventral half of the dorsal funiculus of the spinal cord.

From the level of the rostral pole of the inferior olivary complex, a distinct medial lemniscus could be observed in the ventral aspect of the medial white matter column, lying dorsal to the pyramidal tract. The medial lemniscus was significantly larger than the underlying pyramidal tract but shared a similar shape along its trajectory within the medulla. Within the medial white matter column of the medulla there are likely to be several more ascending or descending pathways, such as the tectospinal tract, but in our material we were not able to distinguish further specific pathways within this column.

The Lateral White Matter Sheath of the Medulla Oblongata. Surrounding the ventral and lateral aspects of the medulla oblongata along its entire length was a sheath of white matter composed of several different pathways, most of which ultimately fed into the inferior cerebellar peduncle (online suppl. fig. AA-XX). Along the ventral aspect of the medulla this white matter sheath was approximately $1 \mathrm{~mm}$ in radial width, but it thickened as it coursed around the lateral margins of the medulla, with the inferior cerebellar peduncle measuring in excess of $5 \mathrm{~mm}$ at its most extensive radial width. Within this sheath, the dorsal and ventral spinocerebellar tracts occupied the majority of the sheath from the level of the spinomedullary junction through to the level of the rostral pole of the nucleus ambiguus. At this level, the olivo- cerebellar tract entered this sheath, enlarging its width. The olivocerebellar tract was seen to emerge from the medial aspect of the inferior olivary nuclear complex, course ventrally in the raphe region as it approached the midline, and decussate and pass under the contralateral inferior olivary complex to join the spinocerebellar tracts within the lateral white matter sheath. Although we could not clearly identify the rostral spinocerebellar and cuneocerebellar pathways, it is this region of the medulla in which they should be located. The majority of these pathways (with the exception of the ventral spinocerebellar tract) feed into the inferior cerebellar peduncle on the lateral aspect of the medulla that passes to the cerebellum. The ventral spinocerebellar tract continues for a few millimetres rostral to this point before passing dorsal and lateral to the spinal trigeminal tract to enter the superior cerebellar peduncle between the inferior cerebellar peduncle and the lateral vestibular nucleus (online suppl. fig. Z, AA).

The Neuromodulatory Nuclei of the Medulla Oblongata

The Catecholaminergic Nuclei of the Medulla Oblongata. Within the medulla of the African elephant we found evidence for six putative catecholaminergic nuclei [composed of tyrosine-hydroxylase-immunopositive $(\mathrm{TH}+)$ neurons], these being: the rostral ventrolateral tegmental group $(\mathrm{C} 1)$, the rostral dorsomedial group $(\mathrm{C} 2)$, the caudal ventrolateral tegmental group (A1), the caudal dorsomedial group (A2), the caudal internuclear catecholaminergic group $(\mathrm{CiN})$ and the area postrema (AP) (online suppl. fig. X-RR). A low density of $\mathrm{TH}+$ neurons forming the $\mathrm{C} 1$ nucleus was found in the ventrolateral medullary tegmentum from the level of the superior olivary nuclear complex to the caudal pole of nucleus ambiguus. These $\mathrm{C} 1$ neurons were located in the most lateral portion of the gigantocellular reticular column (GiCRt) in a position medial to the facial nucleus rostrally and the nucleus ambiguus caudally. The neurons extended dorsally as a column 1-2 cells wide from the very ventral aspect of the GiCRt to approximately the mid-level of the tegmentum. Continuing in the ventrolateral medulla, a column of $\mathrm{TH}+$ neurons located lateral to the nucleus ambiguus in the medial aspect of the parvocellular reticular column (PCRt) and extending to the spinomedullary junction was designated as the $\mathrm{A} 1$ nucleus. The $\mathrm{TH}+$ neurons forming the $\mathrm{A} 1 \mathrm{nu}-$ cleus were located in the ventral half of the PCRt for the majority of the extent of the column, but at more caudal levels this column extended almost through to the central grey matter of the caudal medulla. 
The $\mathrm{TH}+$ neurons assigned to the $\mathrm{C} 2$ nucleus were found to extend from the most rostral portion of the solitary nuclear complex to the level of the caudal pole of the area postrema. These $\mathrm{TH}+$ neurons were found in medium density in the dorsal-most portion of the solitary nuclear mass adjacent to the floor of the fourth ventricle. Within this nucleus there were two clear regional clusters, a rostral densely packed cluster and a caudal region close to the floor of the fourth ventricle termed the dorsal strip. The most rostral portion of the $\mathrm{C} 2$ nucleus was marked by a small densely packed cluster of strongly $\mathrm{TH}+$ cells and neuropil. This rostral portion of $\mathrm{C} 2$ appears to be novel to the elephant, as it has not been observed in the brainstem of other mammals [e.g. Paxinos et al., 2009; Dell et al., 2010]. The dorsal strip of C2 was a tightly packed cluster of $\mathrm{TH}+$ neurons lying close to the floor of the fourth ventricle. TH+ neurons observed at the caudal aspect of the $\mathrm{C} 2$ dorsal strip were seen to spread into the grey matter of the tractus solitarius between the dorsal motor vagal nucleus and the area postrema, forming the $\mathrm{A} 2$ nucleus. The $\mathrm{TH}+$ neurons in the $\mathrm{A} 2$ were less densely packed than in the $\mathrm{C} 2$ nucleus. $\mathrm{TH}$ immunoreactivity also revealed the area postrema (AP), which was observed to be a large cluster of intensely stained, densely packed small neurons found overlying the most lateral portion of the solitary nuclear mass. In the African elephant, the AP extended for a rostrocaudal distance of approximately 15 $\mathrm{mm}$, and was bilaterally paired, being located approximately $5 \mathrm{~mm}$ from the midline and not forming a single midline mass as seen in rodents [e.g. Paxinos et al., 2009; Kruger et al., 2012]. Between the dorsal motor vagal and hypoglossal cranial nerve nuclei, a small number of $\mathrm{TH}+$ neurons represented the $\mathrm{CiN}$ nucleus, although this has not been named in previous studies that have recognized this small TH+ neuronal cluster [Kalia et al., 1984; Weihe et al., 2006]. Some of these CiN neurons were located a small distance into the dorsal caudal medullary tegmentum.

The Serotonergic Nuclei of the Medulla Oblongata. Throughout the entire rostrocaudal extent of the medulla, running from just inferior to the dorsal grey matter to the floor of the medulla, two para-raphe columns, less than 5 cells in width, of medium to large sized cells formed the raphe nuclei. In certain locations, the neurons that formed the raphe nuclei were immunoreactive for serotonin $(5 \mathrm{HT}+)$. These serotonergic immunopositive neurons form what is often referred to as the caudal cluster of serotonergic neurons, and these neurons have axons that project mainly to the spinal cord [Steinbusch, 1981; Törk, 1990]. Within the caudal cluster of serotonergic neurons we found evidence for the raphe magnus ( $\mathrm{RMg}$ ), rostral and caudal ventrolateral columns (RVL and CVL), raphe pallidus $(\mathrm{RPa})$ and raphe obscurus $(\mathrm{ROb})$ nuclei (online suppl. fig. BB-TT). In the rostral medulla, rostral to the inferior olive, bilateral columns of medium to large sized para-raphe $5 \mathrm{HT}+$ neurons formed the raphe magnus nucleus (RMg). This nucleus was observed to span approximately $25 \mathrm{~mm}$ on the rostrocaudal axis.

Within the left and right ventrolateral medullary tegmentum a distinct rostrocaudal column of 5HT+ neurons extending from the level of the facial nucleus to the spinomedullary junction was observed. These have previously been termed the rostral and caudal ventrolateral serotonergic columns [e.g. Maseko et al., 2007; Moon et al., 2007; Dwarika et al., 2008; Dell et al., 2010]. The RVL began as a lateroventral continuation of $5 \mathrm{HT}+$ neurons from the lower portion of the RMg extending over the medial lemniscus and the pyramidal tracts and consolidating as a distinct column lateral to the inferior olivary nuclei on the ventromedial border of the gigantocellular reticular column. The inferior olive topologically distinguishes left and right RVL, and at the level of the nucleus ambiguus the RVL becomes the CVL. The CVL continues in the caudal ventrolateral medullary tegmentum but ends at the level of the caudal pole of the inferior olive. The number of neurons within this RVL/CVL column steadily decreases from rostral to caudal. Although the RVL and CVL are continuous in the African elephant, and indeed in several other eutherian mammals previously studied [e.g. Maseko et al., 2007; Moon et al., 2007; Dwarika et al., 2008; Dell et al., 2010], we make the distinction of two components of these ventrolateral columns, as the caudal portions have not been reported in the opossum or the monotremes [Crutcher and Humbertson, 1978; Manger et al., 2002].

A small number of $5 \mathrm{HT}+$ neurons assigned to the $\mathrm{RPa}$ nucleus were found in the ventral midline of the medulla between with the pyramidal tracts. The RPa was found at the level of the medulla where the caudal pole of the facial nucleus and the rostral pole of the nucleus ambiguus were located. Two loosely arranged bilateral columns of 5HT+ neurons located on either side of the midline from the level of the nucleus ambiguus to the spinomedullary junction were classified as the ROb. In the rostral half of the $\mathrm{ROb}$, the $5 \mathrm{HT}+$ neurons were not restricted to the midline, with some cells being found 1-2 $\mathrm{mm}$ away from the midline, forming a second para-raphe column. Thus, there is a clear central ROb column that extends from the rostral pole of the nucleus ambiguus through to the spinomedullary junction, a distance of $50 \mathrm{~mm}$ on the rostro- 
caudal axis, and a second, lateral column of ROb that was observed from a few millimetres caudal to the most rostral margin of the central column, extending caudally for a distance of approximately $25 \mathrm{~mm}$.

\section{The Intrinsic Nuclei of the Medulla Oblongata}

The Inferior Olivary Nuclear Complex. The inferior olivary nuclear complex is located in the ventromedial rostral half of the medulla, extending over $40 \mathrm{~mm}$ on the rostrocaudal plane, and at its largest it is approximately $15 \mathrm{~mm}$ in diameter (online suppl. fig. FF-QQ). In our material we could readily distinguish four nuclei within this complex: the principal, medial, dorsomedial, and dorsal nuclei. It should be noted that the proportion of the rostral medulla occupied by this nuclear mass appears to be quite large in comparison to other species. Within the nuclear complex, the largest nucleus is the principal olivary nucleus (iopr), which has a rough kidney shape on the coronal plane and is found throughout the entire rostrocaudal extent of the nuclear complex. The medial nucleus (iom) was found between the principal nucleus and the pyramidal tract in the caudal half of the nuclear complex, while slightly dorsal to this position in the rostral half of this complex the dorsomedial nucleus (iodm) was located between the principal nucleus and the raphe. The dorsal nucleus (iod) formed a thin 'cap', or sheet, surrounding the dorsal and lateral aspects of the principal nucleus, forming a dorsolateral border with the medullary gigantocellular reticular nucleus. All of the nuclei within this nuclear complex were populated with a high density of calbindin- and calretinin-immunopositive neurons, but the calbindin-immunopositive neurons had a higher density than the calretinin-immunopositive neurons. From the rostral portion of this nuclear complex, the distinct olivocerebellar tract could be seen to emerge from the medial border of the principal nucleus, gaining fibres as it passed through the dorsal and dorsomedial nuclei, before it turned ventrally at the midline to decussate just dorsal to the pyramidal tract to form the olivocerebellar tract in the ipsilateral, ventrolateral margin of the medulla. This tract was marked by dense clusters of parvalbumin-immunopositive axons that continued around the ventrolateral margin of the medulla to join the inferior cerebellar peduncle.

The Nuclei of Tractus Solitarius. The grey matter forming the nuclear mass of the solitary tract was observed as a rostral continuation of lamina 10 of the spinal cord. This approximately 2 -mm-thick mass of grey matter occupied a position adjacent to the floor of the fourth ventricle and was observed to occur from the caudal pole of the ves-

Elephant Diencephalon and Brainstem tibular nuclei to the spinomedullary junction, spreading up to $10 \mathrm{~mm}$ from the midline in the rostral portions but having a mediolateral dimension of less than $3 \mathrm{~mm}$ in the caudal portions (online suppl. fig. FF-MM). The tractus solitarius was evident as a distinct tract lateral to the grey matter column. For the most part, it was difficult to subdivide this grey matter mass on the basis of the stains used in the present study, although at some levels, for example at the level of the area postrema, several distinct subdivisions were evident. It would be fair to say that further observations with additional stains would reveal the subdivisions seen in other mammals. Invested within this nuclear mass was the dorsal motor vagal nucleus, and medial and ventral to the mass was the hypoglossal nucleus. In the posterior part of the medulla, the mass was bordered laterally and superiorly by the gracile nucleus and tract, and throughout its length it was bordered inferiorly by the medullary reticular nuclei. Our ChAT immunostaining allowed the identification of the preganglionic cells of the inferior salivatory nucleus. These neurons were located in the very rostral aspects of the solitary nuclear mass, rostral to the rostral pole of the dorsal motor vagal nucleus. These neurons likely indicate the gustatory region of the solitary nuclear mass.

The Dorsal Column Nuclei. Within the dorsal funiculus of the spinal cord, two distinct white matter columns, the medially located gracile and the laterally located cuneate fasciculi, were readily identified with myelin staining. At the spinomedullary junction, these fasciculi were observed to invest into the gracile and cuneate nuclei (online suppl. fig. $\mathrm{HH}-\mathrm{XX}$ ). The gracile nucleus was observed to have a rostrocaudal extent of approximately 65 $\mathrm{mm}$, and was seen to occupy a small portion of the middle of the gracile fasciculus in the caudal aspect, but steadily became larger farther rostrally. At its maximum size, the gracile nucleus had a diameter of approximately $3 \mathrm{~mm}$. The cuneate nucleus exhibited similar rostrocaudal dimensions but was slightly smaller $(2.5-\mathrm{mm}$ diameter) than the gracile nucleus due to the presence of the external cuneate nucleus which was also a recipient of the ascending fibres of the cuneate fasciculus. The external cuneate nucleus was located dorsal and lateral to the cuneate nucleus and at first exhibited a lobulated appearance before gradually forming a consolidated nuclear mass. The rostral pole of the external cuneate nucleus was located $30 \mathrm{~mm}$ rostral to the spinomedullary junction and had a rostrocaudal dimension of $35 \mathrm{~mm}$. This nucleus also contains calretinin-immunopositive neurons. The external cuneate nucleus starts $30 \mathrm{~mm}$ rostral to the start of the cuneate nucleus at the spinomedullary 
junction. All three dorsal column nuclei exhibited a low to moderate density of scattered calretinin-immunopositive neurons.

The Potential Neurogenic Zone of the Medulla Oblongata. A thin layer of cells occupying the dorsal medial aspect of the medulla, in direct apposition to the ventricle, appeared to be a continuation of the potential neurogenic zone observed in both the midbrain and the pons (online suppl. fig. X-JJ). This layer of cells was observed to spread up to $5 \mathrm{~mm}$ from the midline and occupied this position from the rostral margin of the medulla through to the level of the posterior pole of the nucleus ambiguus. Again, staining for neurogenic markers such as Ki-67, doublecortin and glial fibrillary acidic protein did not label these cells. Despite this, the appearance and location of this layer of cells indicated that they may be a potential neurogenic site.

The Reticular/Tegmental Nuclei of the Medulla Oblongata

The core of the medulla oblongata is occupied by the reticular formation, which extends from the most rostral level of the medulla through to the spinomedullary junction. As in other mammals, the medullary reticular formation can be subdivided into three major rostrocaudally oriented cell columns: (1) the raphe column, (2) the gigantocellular/magnocellular column (GiCRt), and (3) the parvocellular column (PCRt) (online suppl. fig. Z$\mathrm{UU})$. In addition to these three major cell columns, a caudolaterally located lateral reticular nucleus (LRt) was also observed in the African elephant medulla oblongata. No distinct intermediate reticular nucleus, as reported for the rat [Paxinos et al., 2009], was observed. The raphe column consists of two parasagittal cell columns, often only a few cells wide, located along the entire rostrocaudal extent of the medulla. A thin (less than $1 \mathrm{~mm}$ ), vertically oriented fibre sheet divides the two columns at the midline, and the bilateral columns do not spread more than $2 \mathrm{~mm}$ from the midline at their greatest extent. In several places the cells forming the raphe columns were immunopositive for serotonin (see above), forming individually identifiable nuclei within this column. The remaining neurons of these columns did not exhibit any specific immunoreactivity to the antibodies used in the current study, and thus we could not subdivide this column into more distinct components. At most levels of the medulla the raphe column was clearly isolated from the laterally located GiCRt by a thick column of white matter (incorporating the medial longitudinal fasciculus, medial lemniscus and pyramidal tract, see above).
Lateral to this white matter column, the remaining three subdivisions of the medullary reticular formation were readily distinguishable. The most medial of these three subdivisions was the gigantocellular reticular nucleus, which was readily distinguished from the other reticular nuclei due to the presence of large neurons. The GiCRt had the appearance of a typical mammalian reticular nucleus, with the large cells forming a mesh-like dendritic network around fascicles of the ascending/descending axons. At the lateral margin of the GiCRt, close to its border with the PCRt, a column of tyrosine-hydroxylase-immunopositive neurons, the rostral ventrolateral tegmental group ( $\mathrm{C} 1$, see above), was located. On the medioventral border of this reticular column, the serotonergic rostroventrolateral and caudoventrolateral columns were observed. Occasional calbindin- and calretinin-immunopositive neurons were observed in the GiCRt, and the axons passing through this nucleus were often immunopositive for these two markers.

In a position lateral to the GiCRt and medial to the spinal trigeminal system (see above), another large reticular column, the parvocellular reticular nucleus (PCRt), was located throughout the rostrocaudal extent of the medulla. The border between these two regions was marked by the presence of the nucleus ambiguus and the $\mathrm{TH}+$ rostral ventrolateral tegmental group $(\mathrm{C} 1$, medial to the nucleus ambiguus) and the caudal ventrolateral tegmental nucleus (A1, lateral to the nucleus ambiguus). In addition to this, the marked reduction in size of the soma forming this nucleus provided a reliable delineation of the medial edge of this nucleus. Again, the typical mammalian reticular appearance was present, and occasional calbindin- and calretinin-immunopositive neurons were observed. The axons passing through this nucleus were often immunopositive for these two markers.

The caudolateral aspect of the medullary reticular formation was occupied by the lateral reticular nucleus (LRt). The rostral margins of this nucleus were bordered dorsomedially by the GiCRt and PCRt, but in the more caudal aspects of the LRt the nucleus was distinctly separated from the central reticular core by a distinct column of white matter. The lateral margins of this nucleus abutted against the ascending spinal pathways that formed the lateral wall of the medulla. Apart from being distinct in location, this nucleus had a higher density of medium-sized cells compared with the GiCRt and PCRt, making it readily distinguishable when abutting these nuclei. Despite this higher cell density, the typical reticulated appearance of the nucleus was evident. No calbindin- or calretinin-immunopositive neurons were 
observed in the LRt, but the axons passing through this nucleus were often immunopositive for these two markers.

\section{Discussion}

The current study has provided, for the first time in 50 years [Precechtel, 1925; Abe, 1952; Diepen et al., 1956; Verhaart and Kramer, 1958; Verhaart, 1962, 1963], a comprehensive description of the architectural organization of the diencephalon and brainstem of the African elephant. The acquisition of tissue that was preserved in a manner that allowed investigation with immunohistochemical stains in addition to more traditional cyto- and myelo-architectural stains, from elephants that spent their lives in their natural habitat with little human contact [Manger et al., 2009], ensured our ability to provide a neuroanatomical description of the elephant brainstem that is unparalleled for this iconic African species. While this is clearly advantageous, the sheer size of the combined elephant diencephalon and brainstem means that our description is, for the most part, based on the anatomy found in a single individual [LA1, which had a brain mass of 5,145 g, slightly above the average brain mass of 4,783 g reported for adult elephants by Shoshani et al., 2006]. While we cannot overcome this obstacle (the time and cost required to section and analyse more specimens is prohibitive), we did ensure, using MR imaging, that the gross anatomy of the specimen sectioned was representative of the three specimens obtained, and thus we extrapolate that it was representative of the entire species. In addition to this, the observations made in the current study concur with, and greatly extend, those observations previously made on these structural components of the elephant diencephalon and brainstem [Precechtel, 1925; Abe, 1952; Diepen et al., 1956; Verhaart and Kramer, 1958; Verhaart, 1962, 1963].

As mentioned earlier, the description provided in the current study is based on those descriptions provided in more standard laboratory animals, such as macaque monkeys, cats and laboratory rodents [e.g. Jones, 2007; Paxinos et al., 2009]. The descriptions furnished for these species provided a solid baseline upon which to build the current description, and interestingly, for the most part, it was relatively easy to recognize structures within the elephant diencephalon and brainstem using the published atlases of these more commonly investigated species. The variations from these previous descriptions, where they occurred, were related to four specific aspects of neural information processing: (1) the motor systems,
(2) the auditory and vocalization systems, (3) the orexinergic satiety/wakefulness centre of the hypothalamus, and (4) the potential neurogenic lining of the brainstem. Apart from these specific regions, the remainder of the diencephalon and brainstem of the elephant are what could be considered typically mammalian, with subtle differences in proportions and topology. Due to these observations, this discussion will focus primarily upon the co-ordinated changes within these systems and how they may relate to the life history and observed behaviours of the African elephant.

\section{Motor System Specializations of the African Elephant Brainstem}

The observations made in the current study indicate that a subset of structures involved in the processing of neural information of relevance to the production and co-ordination of movements have specific specializations. Three specific structures exhibited noteworthy differences in organization - the pars compacta of the substantia nigra, the facial nerve nucleus and the inferior olivary nuclear complex. The pars compacta of the substantia nigra (A9pc) exhibited a specific and unusual parcellation of the neurons making up this nucleus; this parcellation of pars compacta neurons extended away from the classical region in which the pars compacta is found (just above the cerebral peduncle), dorsolaterally through the midbrain tegmentum, into the periaqueductal grey matter. No other mammal previously investigated has exhibited such a parcellation and extension of the pars compacta as seen in the current study of the African elephant. Interestingly, no other subdivisions of the substantia nigra, or the adjacent ventral tegmental nuclei, exhibited any morphology that could be considered evidential of specialization. The pars compacta region of the substantia nigra is known to be involved in motor control [e.g. Hodge and Butcher, 1980], and specifically fine motor control [e.g. Pioli et al., 2008], through its projection upon the dorsal striatopallidal complex [Dahlström and Fuxe, 1964]. While we cannot definitively state that the specialized organization of the pars compacta of the substantia nigra in the African elephant will enhance fine motor control, it would appear that this is a likely scenario given the need for fine control of the extensive musculature of the trunk [Endo et al., 2001]. It is possible to speculate that each of the pars compacta islands receives a specific subset of sensory afferents, for example related to trunk flexion, thereby enhancing distinct and discrete trunk movements through a topographically organized striatal dopaminergic projection. 
Both the facial nerve nucleus and the inferior olivary nuclear complex did not exhibit specific specializations through the addition of subdivisions, or anatomical compartmentalization, but both, from a relative, comparative aspect, appear to be quite large. In the case of the facial nerve nucleus, all components of the nucleus appear to be enlarged, indicating an overall increase in the number of axons being sent to the musculature of the cranium under control of this nucleus, and for the most part this would represent the musculature of the trunk. Within the inferior olivary nuclear complex, the principal nucleus of this complex in particular appears to be greatly enlarged. The inferior olivary complex is closely associated with the cerebellum and appears to play a crucial role in both motor learning and motor timing of non-rhythmic movements in the awake animal [De Zeeuw et al., 1998]. This increase in size of the inferior olivary complex appears to be linked to the increased size of the elephant cerebellum [Maseko et al., 2012] and the increased complexity of the cerebellar cortex [Maseko et al., 2013].

It is of interest to note here that the correlated changes in the motor systems in the African elephant brain do not apply to all motor regions of the brain; for example, no clear changes in the ventral anterior nuclei of the dorsal thalamus were obvious. Thus, while changes occur, they appear to be limited in scope and do not involve the entire motor system. This is of interest in terms of the broader aspects of comparative neuroanatomy. Studies of specialized sensory systems across mammals have shown that all parts of the brain involved in processing sensory information reflect the specialization of the sensory system; however, for the motor system in the elephant, only specific regions show specialization. An intriguing aspect of the specializations of the portions of the motor system in the elephant (the pars compacta of the substantia nigra, the inferior olive, the facial nerve nucleus and the cerebellum) is that they all appear to play a key role in the timing and learning of non-rhythmic movements. The specialization of this aspect of movement all points towards control of the movement of the trunk as the corollary of this specialization of a subset of motor-related structures in the elephant brain. The trunk of the African elephant has over 100,000 muscle fibre bundles [Endo et al., 2001] in an unpaired organ that has no skeletal elements. Given this anatomy (in comparison the human has only around 640 muscles in the entire body), the extensive learning required by young elephants to master control of the trunk [Lee and Moss, 1999], and the fact that the elephant uses it trunk extensively and in an intricate manner to manipulate its im- mediate environment, it becomes clear that the neural system involved in the control of timing and learning to use this appendage should be specialized in the elephant. The specialization of only the timing and learning components of the motor system in the elephant can instruct other comparative studies of the motor system in terms of functional interpretation.

\section{Auditory and Vocalization System Specializations of}

the African Elephant Diencephalon and Brainstem

Behavioural and observational studies of the African elephant have revealed the capacity for ultra-low-frequency sound communication [Payne et al., 1986; Garstang, 2004] that is passed as both aerial and seismic sound waves [O'Connell-Rodwell et al., 2006]. O'ConnellRodwell et al. [2006, 2007, 2011] convincingly demonstrated that free-roaming African elephants can detect and react appropriately to the seismic, in addition to the airborne, component of the infrasonic vocalizations produced by other elephants. It is clear that infrasonic vocalizations play a pivotal role in the communication of information between elephants, but currently there are still many significant unanswered questions regarding both the production and the detection of this mode of communication. The current study has revealed at least five regions of the combined vocalization production and auditory/seismic reception system that may provide interesting insights into the developing understanding of infrasonic communication in elephants. For the vocalization aspect, the large and distinct nucleus ellipticus is a clear specialization of the periaqueductal grey matter that we postulate here to be related to infrasonic vocalization production. In terms of reception and interpretation of infrasonic vocalizations, the enlarged lateral superior olivary nucleus and the transverse infrageniculate nucleus appear to be related to the air-borne sound waves, while the enlarged dorsal column nuclei and the ventral posterior inferior nucleus of the dorsal thalamus appear to be related to the seismic aspects of the sound waves.

The neuronal control of vocalization in various mammalian species has been extensively studied, and the neural circuitry that underlies vocalization has been described in detail [reviewed by Jürgens, 1998]. The interesting feature of the mammalian vocal control system of relevance to the elephants is that all telencephalic control of vocalization is channeled to the brainstem through the periaqueductal grey matter. The periaqueductal grey matter, specifically the ventrolateral column, has neurons that when stimulated produce natural-sounding speciesspecific vocalizations [Jürgens, 1994]. It is thought that 
this region of the vertebrate brain controls the activity of the phonatory motoneurons (the nucleus ambiguus) and may act as a vocal pattern generator [Zhang et al., 1994]. It has long been known that the ventrolateral column of the periaqueductal grey matter in the elephant midbrain is specialized as the large nucleus ellipticus [Precechtel, 1925], and the observations in the present study confirm the presence and structure of this nucleus. Given the ability of elephants to create infrasonic vocalizations, it is perhaps not surprising that a specialized structure within the midbrain, in a location likely to represent the ventrolateral column of the periaqueductal grey matter, exists. A similar nucleus is observed in cetaceans [Jansen, 1969; Ridgway, 1990], another group of species with a specialized vocal production system. How this system interacts with the cranial and thoracic morphology of the elephant to create the infrasonic vocalizations is currently unknown, but a recent study demonstrated that the laryngeal vocal folds are capable of vibrating at frequencies similar to those of the infrasonic vocalizations [Herbst et al., 2012]. It would be safe to assume, though, that the production of infrasonic vocalizations in elephants is likely to have a complex neural substrate and that the nucleus ellipticus may form a key processing station in vocal production.

The medial geniculate body is the auditory processing station of the dorsal thalamus, taking information from the inferior colliculus to the auditory cortex. In general terms, the medial geniculate body of the African elephant has a nuclear parcellation that resembles that seen in most mammals [Jones, 2007; Paxinos et al., 2009]; however, as noted previously [Precechtel, 1925; Abe, 1952], an additional nucleus located on the ventrolateral surface of the medial geniculate body, the transverse infrageniculate nucleus (TIN), is present in the elephant. Such a nucleus has not been observed in other mammals studied to date [Jones, 2007]. Precechtel [1925] and Abe [1952] indicated that this nucleus is laminated, and the current study lends some support to this conclusion. In the current study, the Nissl and myelin staining did not reveal a distinct laminar pattern in the TIN, but the immunostaining for calbindin and calretinin revealed an incipient bilaminar appearance, with calbindin neurons roughly limited to the ventrolateral lamina of the nucleus and calretinin neurons being limited to the mediodorsal lamina of the nucleus. Thus, this novel medial geniculate nucleus also shows a degree of anatomical lamination, suggestive of specialized processing of incoming neural information. Studies of the tonotopic arrangement of the mammalian ventral medial geniculate nucleus (MGv), which in the elephant is dorsomedially adjacent to the TIN, have shown that the ventrolateral edge of the MGv is the region where the lowest tones received are processed [Imig and Morel, 1985; Jones, 2007]. The consistent observation of the processing of lower frequencies in the ventrolateral portion of the MGv in other mammals suggests that the TIN in the elephant is a specialized portion of the MGv specifically tuned to process infrasound. This suggestion is obviously speculative but, given the likelihood that physiological recording will never be able to be undertaken in the TIN, this educated guess probably represents the best explanation of the function of this nucleus.

Within the ventrolateral pons the superior olivary complex, an integral processing station of the ascending auditory system, had an overall nuclear organization resembling that seen in the laboratory rat [Paxinos et al., 2009]; however, the entire nuclear complex was dominated by an enlarged lateral superior olivary nucleus [Glendenning and Masterton, 1998]. While in general the superior olivary nuclear complex is involved in neural processing related to sound localization, the different components of this nuclear complex undertake different roles to achieve this task. The lateral superior olivary nucleus is specifically involved in using interaural differences in sound intensity to localize the sound source [Tsuchitani and Boudreau, 1967]. The enlargement of the lateral superior olivary nucleus would suggest that interaural sound intensity differences are of most importance to the elephant for sound localization.

All three dorsal column nuclei (gracile, cuneate and external cuneate) appear to be comparatively large in the African elephant. The dorsal column nuclei process neural information carried from receptors in the skin sensitive to light touch and proprioception. Given the pachydermatous nature of the skin of the elephant, it seems unlikely that light or fine touch is a central aspect of the sensitivity of the elephant skin; however, several reports have shown that both the tip of the trunk [Smith, 1890; Rasmussen and Munger, 1996] and the feet [Bouley et al., 2007] are invested with numerous Pacinian corpuscles, well known for their sensitivity to vibrotactile stimuli [Horch, 1991]. It has been postulated that the pacinian corpuscles in the elephant feet, and likely the trunk, can serve to detect the seismic component of the infrasonic vocalizations [Bouley et al., 2007]. The presence of a distinct ventral posterior inferior nucleus of the dorsal thalamus, thought to process pacinian-like input specifically [Dykes et al., 1981], and not clearly present in all mammals [Jones, 2007], supports the notion that a specialized pathway for vibratory reception from peripheral Pacinian 
corpuscles is present in the elephant brain. Given the paucity of other tactile receptors across the elephant skin surface, it seems likely that the size of the dorsal column nuclei and the presence of the ventral posterior inferior nucleus of the dorsal thalamus are a central nervous system reflection of the pacinian tactile sensory specializations of the trunk and the feet.

O'Connell-Rodwell et al. [2006] convincingly demonstrated that free-roaming African elephants can detect, and react appropriately to, the seismic component of the infrasonic vocalizations produced by other elephants. While O'Connell-Rodwell et al. [2007, 2011] appear to feel that elephants can detect the semantic component of infrasonic vocalizations through the detection of seismic waves, we feel this conclusion may have been reached in haste. The heterogeneous nature of the ground, and the extended distance through which the vocalization must travel, is likely to confound or muffle the semantic contents of the vocalization. One interesting aspect of the seismic waves that has not been examined to date is the fact that seismic waves travel at approximately $5,000 \mathrm{~m} / \mathrm{s}$, far quicker than airborne sound waves, which travel at around $350 \mathrm{~m} / \mathrm{s}$. This would, for example, mean that if the source of the infrasonic vocalization were $1,000 \mathrm{~m}$ from the detector, the seismic component of the infrasonic vocalization would arrive at the detector in $200 \mathrm{~ms}$, while the aerial wave would arrive at the detector around $2,600 \mathrm{~ms}$ later. This differential in arrival of the two waves allows for the detector to determine, through experiential learning, the approximate distance of the sound source from its location. We can posit the following scenario as an example. Let us imagine that a subordinate male is drinking at a water hole and he detects the infrasonic vocalization of a dominant male. He may pause his drinking and adopt the 'listening' posture commonly seen in elephants [O'Connell-Rodwell et al., 2006]. If the airborne component of the vocalization arrives $2,600 \mathrm{~ms}$ after the seismic component, he will know the dominant male is at least $1,000 \mathrm{~m}$ away and will likely relax and continue drinking; however, if the airborne wave were to arrive $260 \mathrm{~ms}$ after the seismic wave, he will know the dominate male is only approximately $100 \mathrm{~m}$ away. In order to avoid a potentially dangerous confrontation, the subordinate male may then choose to leave the water hole. By determining the time delay between the arrivals of the two wave types, a relatively simple task for associative cerebral cortex, elephants may be able to know, roughly, the location of other elephants in the region, up to distances as far as $5 \mathrm{~km}$ away. If this possibility turns out to be correct, this would provide explanations for many elephant behaviours, including the 'listening' behaviour, and the seemingly uncanny ability of foraging elephant herds to maintain a consistent distance between each other.

Thus, based on a combination of anatomical and neuroanatomical studies, we can propose a mechanism whereby the elephants can use infrasound sensitivity to determine the location of the sound source and the information carried in infrasonic vocalizations. Upon sensing the seismic component of the infrasonic vocalization through the pacinian corpuscles and neural processing in the dorsal column and ventral posterior inferior nucleus of the dorsal thalamus, the elephant can adopt the 'listening' posture until such time as the airborne component of the vocalization arrives. By paying attention to the differential in arrival of the two wave types, the elephant may be provided with the information needed to determine the approximate distance of the sound source. Using the enlarged lateral superior olivary nucleus, the elephant, through interaural intensity differences, will be able to determine the approximate direction of the sound source. Thus, the elephant can, through combined tactile and auditory processing, detect the distance and direction of sound sources. A specialized nucleus within the medial geniculate body, the transverse medial geniculate body, could then interact with the cerebral cortex to extract the semantic information carried in the airborne component of the infrasonic vocalization. Based on distance, direction, and semantics of the detected infrasonic vocalization, the elephant may then make a decision on the appropriate behaviour in relation to the detected vocalization. Obviously, behavioural studies would be required to support or negate this proposed mechanism of infrasonic vocalization detection and use; however, given the current observations, this mechanism is a likely candidate to explain many behaviours of freeroaming elephants related to infrasonic communication.

\section{The Satiety/Wakefulness Centres of the Hypothalamus and Pons}

The orexinergic system within the hypothalamus and the noradrenergic locus coeruleus in the periventricular grey matter of the pons both showed distinct specializations in the brain of the African elephant. The mammalian orexinergic system has been implicated in the regulation of blood pressure, neuroendocrine functions, body temperature, the promotion of wakefulness, stimulation of food intake, energy homeostasis and locomotor activity [e.g. Peyron et al., 1998; Wagner et al., 2000; McGranaghan and Piggins, 2001; Fabris et al., 2004; Baumann and Bassetti, 2005; Spinazzi et al., 2006; McGregor 
et al., 2011]. The locus coeruleus is known to be a powerful wake-promoting centre of the brain through its projection to many subcortical structures that activate noradrenergic receptors [Berridge et al., 2012].

Within the orexinergic system of the hypothalamus, the elephant displayed a medial hypothalamic parvocellular cluster of neurons, in addition to the magnocellular clusters typical of mammals located in the lateral hypothalamus [Dell et al., 2012]. The only other species in which this parvocellular orexinergic neuronal cluster has been observed are the giraffe and the harbour porpoise, both members of the Cetartiodactyl order [Dell et al., 2012]. Dell et al. [2012] considered that this medial parvocellular cluster of orexinergic neurons may be involved in either appetitive drive or the promotion of wakefulness in both species investigated, as both species source highnutrient food, and both have specialized sleep needs. In terms of the appetitive drive of the elephant, it is well known that they can consume up to $500 \mathrm{~kg}$ of low-nutrient foliage per day. Thus, an additional orexinergic nucleus, specifically devoted to increasing their appetitive drive, may prove to be very useful for such a large animal requiring such a large quantity of food each day. Alternatively, the elephant needs to be awake for a long period in order to consume this much food; thus, an additional orexinergic nucleus promoting longer wakefulness would also be of use. In captivity, where food is provided ad libitum, elephants are thought to sleep for 4-6 h/day [Tobler, 1992]; however, unpublished studies of the rest and activity of elephants in the wild have demonstrated that they likely sleep for less than $3 \mathrm{~h} /$ day [Manger, Lyamin, Siegel, pers. commun.]. At this stage it would appear that either explanation for the presence of an additional cluster of orexinergic neurons in the medial hypothalamus has value, but perhaps the two concepts are inseparable and the combined needs of increased appetitive drive and increased wakefulness explain the presence of this nucleus not only in the elephant but also in the giraffe and harbour porpoise.

The observation of an additional subdivision of the locus coeruleus, that is termed the A6 medial subdivision herein, is also of interest as the locus coeruleus is a powerful promoter of wakefulness. The locus coeruleus also receives substantial input from the orexinergic neurons of the hypothalamus [Sutcliffe and de Lecea, 2000]. In the elephant, the main nucleus of the locus coeruleus displays the typically diffuse packing of neurons seen in most mammals [Dell et al., 2010; Kruger et al., 2012] but, unlike the other Afrotherians previously studied, the rock hyrax, golden mole and elephant shrew [Gravett et al., 2009;
Calvey et al., 2013], this nucleus has a large number of neurons. In addition, a novel small cluster of neurons was located medial to this main cluster. This more complex arrangement of the locus coeruleus in the elephant would appear to support the concept that the drive for wakefulness is strong in the elephant, allowing it time to consume the large daily quantity of low-quality foliage required to sustain its large body mass. Moreover, the A7 nucleus appeared to be made up of a large number of cells, and previous studies have implicated the A7 nucleus in feeding [McCabe et al., 1984]. A detailed study of the neural systems related to both the sleep-wake cycle and satiety would be of interest to pursue for the elephant, as these two important factors in the life of the elephant, and indeed all mammals [Sutcliffe and de Lecea, 2000], seem to be inextricably linked.

\section{The Potential Neurogenic Lining of the Brainstem}

One of the more intriguing observations made in the course of the current study was the appearance of a thin lamina of cells lining the cerebral aqueduct, the floor of the fourth ventricle, and the dorsolateral margin of the midbrain over the colliculi which has the appearance of a development neurogenic zone. Indeed, within the midbrain, this lining was thicker and formed a significant bulge that extended into the periaqueductal grey matter. In attempting to determine the identity of this layer of cells, we tried immunostaining for Ki67, doublecortin (DCX) and glial fibrillary acidic protein (GFAP); however, no immunoreactivity could be detected with these stains. While immunostaining for Ki67 antibody does not appear to be reactive to elephant tissue, both the DCX and GFAP antibodies used were immunoreactive in the elephant olfactory bulb [Ngwenya et al., 2011]. The lack of staining for these three antibodies in this region indicates that perhaps it is not neurogenic but plays some other role; however, it would be important to investigate this region further with a wider range of antibodies related to neurogenesis. The current study also demonstrates that these cells are not immunoreactive for choline acetyltransferase, tyrosine hydroxylase, serotonin, orexin-A, parvalbumin, calbindin or calretinin. Thus, through a process of elimination, they are either excitatory neurons or have some specific function that we are currently unaware of, although the neurogenic function would appear to be the most reasonable explanation given the location and appearance of this region of cells.

The elephant used for this range of immunostains, LA1, was 20-25 years old. Male African elephants reach puberty at approximately 10 years of age but do not be- 
come socially mature, i.e. old enough to compete for oestrus females, until they reach 35 years, and they can live to an estimated 60 years in their natural habitat [Skinner and Chimimba, 2005]. Thus, while not a fully mature bull elephant, LA1 is clearly an adult. Despite this, the observation of the potential neurogenic lining may indicate that this elephant is still undergoing normal brain growth. In order to test this, we were provided with thioninstained sections of the midbrain of two further elephants: a 40-year-old female African elephant and a 48-year-old female Asian elephant (courtesy of Prof. Jerry Siegel), both of which evinced the clear bulge of cells adjacent to the cerebral aqueduct in the periaqueductal grey matter. These additional observations indicate that this lining, whatever its nature, is likely to be a neural character common to all extant elephants. In this sense understanding what this structure is and does becomes even more interesting; however, at this stage we can only conclude that further investigation is required to elucidate this matter.

\section{Stasis in the Evolution of the Mammalian}

\section{Diencephalon and Brainstem}

It is important to note that for the vast majority of the current study we could use published atlases and descriptions of commonly studied mammals to both determine and understand the anatomical configuration of the diencephalon and brainstem in the African elephant. The variances from these descriptions (noted and discussed above), while of interest to understanding specific aspects of the elephant, were actually quite few. It has long been a tenet in comparative neuroanatomical studies that larger brains are generally more complexly organized than smaller brains in terms of the number of subdivisions of nuclei in different neural systems [e.g. Stephan et al., 1981]. The current study, which outlines the organization of the diencephalon and brainstem in the largest extant terrestrial brain, contradicts this principle. It is without a doubt generally accepted that evolutionary changes in the structure of the diencephalon and brainstem will be relatively fewer than in regions of the telencephalon due to the fact that making changes to an established and evolutionarily successful system is likely to result in lethal phenotypes. Despite this, the complexity of the organization of the nuclei within the dorsal thalamus is also thought to be an accurate proxy of the complexity of organization of the cerebral cortex. Apart from the clear addition of the medial geniculate leaflet, the organization of the African elephant dorsal thalamus, at least at the level investigated herein, is not in any way particularly more or less complex than seen in other mammals [Jones, 2007]. Moreover, this generalized mammalian organization of the dorsal thalamus indicates that the cerebral cortex of the African elephant likely does not have a more complex areal organization than other mammals despite its large size. Similar conclusions can be drawn about neural systems within the brainstem, including those we identified with immunohistochemical stains - while there are some minor changes in the nuclear organization of these systems, for the most part they are similar to that seen in other mammals [Manger, 2005; Dell et al., 2010; Kruger et al., 2012]. In fact, it would be fair to say that the elephant brainstem is, for the most part, a roughly scaled up version of the laboratory rat brainstem [Paxinos et al., 2009].

These observations indicate that, at the level of analysis undertaken herein, evolutionary changes in the organization of the mammalian brainstem are likely to be few and will often be related to specific unique behaviours that characterize a particular species or group of closely related species as there are clear phylogenetic links between many observed changes [Manger, 2005; Maseko and Manger, 2007; Maseko et al., 2007; Dell et al., 2010; Kruger et al., 2012]. This evolutionary stasis in the organization of the brainstem leads to two possible conclusions: (1) it is very difficult for evolutionary changes in the nuclear organization of the brainstem to occur without the production of lethal phenotypes or (2) the level of analysis undertaken here is not fine enough in detail to extract much of the phylogenetic variance in the organization of the brainstem. Indeed, the latter conclusion, while not negating the first, indicates that finer levels of organization, such as at the level of the individual neuron, the chemical anatomy of neurons, receptors, molecular organization, or even active gene expressions, are potentially where important changes that may affect the overall behaviour of animals are likely to occur. Unfortunately, obtaining the material required for investigations of these levels of neuronal organization in the African elephant is not easy, but we can safely conclude that at the level of nuclear organization, as investigated here, there is a very robust organization across mammalian species that is not readily amenable to evolutionary change [Manger, 2005].

\section{Thalamic Parcellation and Neocortical Parcellation - Correlations in the Elephant?}

It is a common tenet in the comparative neurosciences, though often not overtly stated, that the degree of nuclear parcellation in the dorsal thalamic nuclear mass is reflected in the degree of parcellation of neocortical areas. Jones [2007] is one of the few who has made direct statements about this potential correlation, indicating that 
species with complexly organized lateral geniculate nuclei generally have more visual cortical areas, arguing that the highly parcellated human thalamus is [Jones, 2007, p. 84] 'a reflection of the equally high degree of structural and functional parcellation of the ... cortex.... Monotony of cortex breeds monotony of thalamus (or is it vice versa?).' The current study of the African elephant dorsal thalamic nuclear mass may thus provide some predictions about how the cerebral cortex of this species would be organized, i.e. do elephants have a highly parcellated cerebral cortex? This is an interesting question to tackle, as it is generally considered that species with highly parcellated cerebral cortices show greater cognitive flexibility. This is based on the assumption that a greater number of cortical areas leads to an increase in the degrees of freedom for neuronal processing, which in turn allows more complex processing to occur, thus providing a basis for increased behavioural flexibility.

In the current study we followed the schema provided by Jones [2007] as our base to describe the elephant dorsal thalamic nuclear mass. Our observations indicate that only two regions of the elephant dorsal thalamic nuclear mass exhibit increased parcellation in comparison to other mammals that may reflect additional cortical regions - the medial nuclear complex, and the medial geniculate complex. In the medial nuclear complex, we observed that the mediodorsal nucleus was clearly segregated into three subdivisions, a condition that is usually only clearly seen in the brains of primates, although there are indications of these divisions even in the rat brain [Jones, 2007]. The mediodorsal nucleus is strongly interconnected with the prefrontal cortex (that rostral to the premotor cortex) of mammals. The prefrontal cortex is known to be involved in cognition, learning and memory, tracking of behavioural conditions in relation to a range of potential responses, working memory, and complex olfactionbased behaviours, amongst many others. African elephants are known to have a large and complexly organized olfactory bulb [Ngwenya et al., 2011]; thus, the prefrontal cortex of elephants, along with the mediodorsal nucleus, may have become more parcellated in response to increased olfactory sensitivity in elephants. Moreover, the prefrontal cortex was the location where pyramidal neurons exhibiting large and complex dendritic arbors (matriarch neurons), rivalling those seen in human prefrontal cortex, were observed in the African elephant [Jacobs et al., 2011]. Thus, it is possible that the prefrontal cortical region of the elephants is a region that is highly parcellated and may indeed subserve many of the complex behaviours exhibited by elephants.

Elephant Diencephalon and Brainstem
The additional nucleus in the medial geniculate complex, the transverse infrageniculate nucleus (TIN), possibly involved in the processing of low-frequency sounds (see above), may be another feature of the dorsal thalamic nuclear mass leading to increased cortical parcellation. It is unclear whether TIN is a novel nucleus or a specialized part of the ventral medial geniculate nucleus. If the latter is true, it is possible that additional novel areas have not been added to the auditory cortex but that existing areas have regions of specialization; however, if this is a novel nucleus, then it is possible that additional auditory cortical areas have been added. Either way, it is clear that the cortical processing of auditory information in the elephant is likely to be more complex than seen in many other mammals. Although we could not identify specific subdivisions of the lateral posterior-pulvinar complex, this complex occupied a large portion of the dorsal thalamic nuclear mass in the elephant. It is likely that several individual nuclei are housed within this complex, and that there may be novel nuclei in this region. If this is the case, then the cortical target region of the LP-pulvinar complex of the elephant is likely to be highly parcellated, as is seen in the target region of this complex in primates. Despite these possibilities, the remainder of the dorsal thalamic complexes in the elephant were not specialized above and beyond that seen in many mammalian species, even those with small brains. Thus, despite the generally large size of the elephant cortical sheet, it may not be extensively parcellated and may indeed have a more homogeneous appearance than one would predict based on comparisons to other mammals - maybe larger brains are not always highly parcellated. The parcellation of elephant cerebral cortex is a significant target for future study.

\section{Future Directions}

The current study has, for the first time in over 50 years [Cozzi et al., 2001], provided a comprehensive framework detailing the anatomical organization of the African elephant diencephalon and brainstem. The analysis provided here has shown that specific aspects of the organization of these brain regions in the elephant have undergone a moderate amount of change, specifically those associated with the timing aspects of the motor systems, the production and reception of vocalizations, the satiety and sleep-wake systems, and the potential neurogenic lining of much of the ventricular surface in the brainstem. All of these aspects of change are worth investigating in greater detail, with a greater range of techniques where possible. These regions of change appear to be related to specific 
behaviours unique to the elephants and thus are of importance in gaining an overall understanding of the way the brain processes information of relevance to these unique behaviours.

Whilst the study of these important variances will undoubtedly lead to a better understanding of elephant behaviour, the regions that do not vary considerably should not be overlooked, as subtle changes may indeed play an important role in elephant behaviour. While it is reasonably easy to conclude that the organization of the elephant brainstem is mostly typical of that of mammals in general, there are many subtle changes that have not been strongly expressed in the current study. Subtle variations in the organization of specific nuclei, such as the salivatory nuclei, may be observed on closer inspection and comparison to a broader range of species. This is just an example, but it may apply across many of the co-ordinated neural systems found in the diencephalon and brainstem. Only a very broad-based and detailed comparative assessment could define the subtle changes in the anatomy of these brain regions and how they relate to overt elephant behaviour. However, one could, for example, take a specific region, such as the inferior colliculus, which in the elephant for all practical purposes looks the same as a rat inferior colliculus, and investigate this in greater detail and it is likely that subtle differences related to the differing auditory worlds, and the manner in which they topographically map these worlds, would emerge. When approached from this aspect, there is an enormous amount of work to be undertaken in the future in order to provide a comprehensive picture of how the elephant brain produces elephant behaviour.

\section{Acknowledgments}

This study was supported by a grant from the South African National Research Foundation to P.R.M. (grant No. FA2005033100004). We would like to thank Dr. Theo Nel and staff at Wits-DGMC for the kind use of the MR scanner and their help during this project, Dr. Hilary Madzikanda of the Zimbabwe Parks and Wildlife Management Authority, and Dr. Bruce Fivaz and the team at the Malilangwe Trust, Zimbabwe. Lastly, P.R.M. wishes to dedicate this paper to the memory of Edward (Ted) G. Jones, with whom he had planned to undertake the work on the dorsal thalamus presented here for many years, but which they never managed to find the time to do together - his input to this study was sorely missed, as is he.

\section{References}

Abe Y (1952): Zur Cytoarchitektonik des Thalamus beim Elefanten. Folia Psychiatr Neurol Jpn 5:213-239.

- Asher RJ, Bennett N, Lehmann T (2009): The new framework for understanding placental mammal evolution. Bioessays 31:853-864.

-Baumann CR, Bassetti CL (2005): Hypocretins (orexins): clinical impact of the discovery of a neurotransmitter. Sleep Med Rev 9:253-268.

- Berridge CW, Schmeichel BE, Espana RA (2012): Noradrenergic modulation of wakefulness/ arousal. Sleep Med Rev 16:187-197.

Bhagwandin A, Fuxe K, Bennett NC, Manger PR (2008): Nuclear organization and morphology of cholinergic, putative catecholaminergic and serotonergic neurons in the brains of two species of African mole-rat. J Chem Neuroanat 35:371-387.

- Bhagwandin A, Fuxe K, Bennett NC, Manger PR (2011): Distribution of orexinergic neurons and their terminal networks in the brains of two species of African mole rats. J Chem Neuroanat 41:32-42.

Bjarkam CR, Sorensen JC, Geneser FA (1997): Distribution and morphology of serotoninimmunoreactive neurons in the brainstem of the New Zealand white rabbit. J Comp Neurol 380:507-519.
Bouley DM, Alarcon CN, Hildebrandt T, O'Connell-Rodwell CE (2007): The distribution, density and three-dimensional histomorphology of Pacinian corpuscles in the foot of the Asian elephant (Elephas maximus) and their potential role in seismic communication. J Anat 211:426-435.

Calvey T, Patzke N, Kaswera C, Gilissen E, Bennett NC, Manger PR (2013): Nuclear organisation of some immunohistochemically identifiable neural systems in three Afrotherian species - Potomogale velox, Amblysomus hottentotus and Petrodromus tetradactylus. J Chem Neuroanat 50-51:48-65.

Cozzi B, Spagnoli S, Bruno L (2001): An overview of the central nervous system of the elephant through a critical appraisal of the literature published in the XIX and XX centuries. Brain Res Bull 54:219-227.

Crutcher KA, Humbertson AO (1978): The organization of monoamine neurons within the brainstem of the North American opossum (Didelphis virginiana). J Comp Neurol 179:195-222.

Dahlström A, Fuxe K (1964): Evidence for the existence of monoamine-containing neurons in the central nervous system. 1. Demonstration of monoamine in the cell bodies of brainstem neurons. Acta Physiol Scand 62:1-52.
Dell LA, Kruger JL, Bhagwandin A, Jillani NE, Pettigrew JD, Manger PR (2010): Nuclear organization of cholinergic, putative catecholaminergic and serotonergic systems in the brains of two megachiropteran species. J Chem Neuroanat 40:177-195.

Dell LA, Patzke N, Bhagwandin A, Bux F, Fuxe K Barber G, Siegel JM, Manger PR (2012): Organization and number of orexinergic neurons in the hypothalamus of two species of Cetartiodactyla: a comparison of giraffe (Giraffa camelopardalis) and harbour porpoise (Phocoena phocoena). J Chem Neuroanat 44:98-109.

De Zeeuw CI, Simpson JI, Hoogenraad CC, Galjart N, Koekkoek SKE, Ruigrok TJH (1998): Microcircuitry and function of the inferior olive. Trends Neurosci 21:391-400.

Diepen R, Janssen P, Engelhardt F, Spatz H (1956): Recherches sur le cerveau de l'elephant d'Afrique (Loxodonta africana Blum): donnees sur l'hypothalamus. Acta Neurol Belg 11: 759-788.

Dwarika S, Maseko BC, Ihunwo AO, Fuxe K, Manger PR (2008): Distribution and morphology of putative catecholaminergic and serotonergic neurons in the brain of the greater cane rat, Thryonomys swinderianus. J Chem Neuroanat 35:108-122. 
Dykes RW, Sur M, Merzenich MM, Kaas JH, Nelson RJ (1981): Regional segregation of neurons responding to quickly adapting, slowly adapting, deep and Pacinian receptors within thalamic ventroposterior inferior nuclei in the squirrel monkey (Saimiri sciureus). Neuroscience 6:1687-1692.

Endo H, Hayashi Y, Komiya T, Narushima E, Sasako M (2001): Muscle architecture of the elongated nose in the Asian elephant (Elephas maximus). J Vet Med Sci 63:533-537.

-Fabris C, Cozzi B, Hay-Schmidt A, Naver B, Moller M (2004): Demonstration of an orexinergic central innervaton of the pineal gland of the pig. J Comp Neurol 471:113-127.

Gallyas F (1979): Silver staining of myelin by means of physical development. Neurol Res 1:203-209.

-Garstang M (2004): Long-distance, low-frequency elephant communication. J Comp Physiol A Neuroethol Sens Neural Behav Physiol 190: 791-805.

Glendenning KK, Masterton RB (1998): Comparative morphometry of mammalian central auditory systems: variation in nuclei and form of the ascending system. Brain Behav Evol 51: 59-89.

Gravett N, Bhagwandin A, Fuxe K, Manger PR (2009): Nuclear organization and morphology of cholinergic, putative catecholaminergic and serotonergic neurons in the brain of the rock hyrax, Procavia capensis. J Chem Neuroanat 38:57-74.

Gravett N, Bhagwandin A, Fuxe K, Manger PR (2011): Distribution of orexin-A immunoreactive neurons and their terminal networks in the brain of the rock hyrax, Procavia capensis. J Chem Neuroanat 41:86-96.

-Herbst CT, Stoeger AS, Frey R, Lohscheller J, Titze IR, Gumpenberger M, Fitch WT (2012): How low can you go? Physical production mechanism of elephant infrasonic vocalizations. Science 337:595-599.

Hodge GK, Butcher LL (1980): Pars compacta of the substantia nigra modulates motor activity but is not involved importantly in regulating food and water intake. Naunyn Schmiedebergs Arch Pharmacol 313:51-67.

-Hökfelt T, Johansson O, Fuxe K, Goldstein M, Park D (1976): Immunohistochemical studies on the localization and distribution of monoamine neuron systems in the rat brain. 1. Tyrosine hydroxylase in the mes- and diencephalon. Med Biol 54:427-53.

Hökfelt T, Martenson R, Björklund A, Kleinau S, Goldstein M (1984): Distributional maps of tyrosine-hydroxylase-immunoreactive neurons in the rat brain; in Björklund A, Hökfelt T (eds): Handbook of Chemical Neuroanatomy: Classical Neurotransmitters in the CNS, Part 1. Amsterdam, Elsevier, vol 2, pp 277-379.

Horch K (1991): Coding of vibrotactile stimulus frequency by Pacinian corpuscle afferents. J Acoust Soc Am 89:2827-2836.

-Imig TJ, Morel A (1985): Tonotopic organization in ventral nucleus of medial geniculate body in the cat. J Neurphysiol 53:309-340.
Jacobs B, Lubs J, Hannan M, Anderson K, Butti C, Sherwood C, Hof PR, Manger PR (2011): Neuronal morphology in the African elephant (Loxodonta africana) neocortex. Brain Struct Funct 215:273-298.

Jansen J (1969): On cerebellar evolution and organization from the point of view of a morphologist; in Llinás R (ed): Neurobiology of Cerebellar Evolution and Development. Chicago, American Medical Association Education and Research Foundation, pp 881-893.

Jones EG (2007): The Thalamus. Cambridge, Cambridge University Press.

Jürgens U (1994): The role of the periaqueductal gray in vocal behavior. Behav Brain Res 62: $107-117$.

Jürgens U (1998): Neuronal control of mammalian vocalization, with special reference to the squirrel monkey. Naturwissenschaften 85 : 376-388.

Kalia M, Fuxe K, Goldstein M, Harfstrand A, Agnati LF, Coyle JT (1984): Evidence for the existence of putative dopamine-, adrenalineand noradrenaline-containing vagal motor neurons in the brainstem of the rat. Neurosci Lett 50:57-62.

-Kitahama K, Geffard M, Okamura H, Nagatsu I, Mons N, Jouvet M (1990): Dopamine- and dopa-immunoreactive neurons in the cat forebrain with reference to tyrosine hydroxylase-immunohistochemistry. Brain Res 518: 83-94.

-Kitahama K, Sakamoto N, Jouvet A, Nagatsu I, Pearson J (1996): Dopamine-beta-hydroxylase and tyrosine hydroxylase immunoreactive neurons in the human brainstem. J Chem Neuroanat 10:137-146.

Klemm WR, Vertes RP (1990): Brainstem Mechanisms of Behavior. New York, Wiley-Interscience.

Kozicz T, Bittencourt JC, May PJ, Reiner A, Gamlin PD, Palkovits M, Horn AK, Toledo CA, Ryabinin AE (2011): The Edinger-Westphal nucleus: a historical, structural, and functional perspective on a dichotomous terminology. J Comp Neurol 519:1413-1434.

Kruger JL, Dell LA, Bhagwandin A, Jillani NE, Pettigrew JD, Manger PR (2010): Nuclear organization of cholinergic, putative catecholaminergic and serotonergic systems in the brains of five microchiropteran species. J Chem Neuroanat 40:210-222.

Kruger JL, Patzke N, Fuxe K, Bennett NC, Manger PR (2012): Nuclear organization of cholinergic, putative catecholaminergic, serotonergic and orexinergic systems in the brain of the African pygmy mouse (Mus minutoides): organizational complexity is preserved in small brains. J Chem Neuroanat 44:45-56.

Kubota K (1967): Comparative anatomical and neurohistological observations on the tongues of elephants (Elephas indicus and Loxodonta africana). Anat Rec 157:505-515.
Lee PC, Moss CJ (1999): The social context for learning and behavioral development among wild African elephants; in Box HO, Gibson KR (eds): Mammalian Social Learning: Comparative and Ecological Perspectives. Cambridge, Cambridge University Press, pp $102-$ 125 .

Limacher AM, Bhagwandin A, Fuxe K, Manger PR (2008): Nuclear organization and morphology of cholinergic, putative catecholaminergic and serotonergic neurons in the brain of the Cape porcupine (Hystrix africaeaustralis): increased brain size does not lead to increased organizational complexity. J Chem Neuroanat 36:33-52.

Manger PR (2005): Establishing order at the systems level in mammalian brain evolution. Brain Res Bull 66:282-289.

- Manger PR, Fahringer HM, Pettigrew JD, Siegel JM (2002): Distribution and morphology of serotonergic neurons in the brain of the monotremes. Brain Behav Evol 60:315-332.

- Manger PR, Hemingway J, Haagensen M, Gilissen E (2010): Cross-sectional area of the elephant corpus callosum: comparison to other eutherian mammals. Neuroscience 167:815824.

- Manger PR, Pillay P, Maseko BC, Bhagwandin A, Gravett N, Moon D, Jillani NE, Hemingway J (2009): Acquisition of the brain of the African elephant (Loxodonta africana): perfusion-fixation and dissection. J Neurosci Methods 179: $16-21$.

-Manger PR, Prowse M, Haagensen M, Hemingway J (2012): Quantitative analysis of neocortical gyrencephaly in African elephants (Loxodonta africana) and six species of cetaceans: comparison with other mammals. J Comp Neurol 520:2430-2439.

Maseko BC, Bourne JA, Manger PR (2007): Distribution and morphology of cholinergic, putative catecholaminergic and serotonergic neurons in the brain of the Egyptian Rousette flying fox, Rousettus aegyptiacus. J Chem Neuroanat 34:108-127.

-Maseko BC, Jacobs B, Spocter MA, Sherwood CC, Hof PR, Manger PR (2013): Qualitative and quantitative aspects of the microanatomy of the African elephant cerebellar cortex. Brain Behav Evol 81:40-55.

Maseko BC, Manger PR (2007): Distribution and morphology of cholinergic, catecholaminergic and serotonergic neurons in the brain of Schreiber's long-fingered bat, Miniopterus schreibersii. J Chem Neuroanat 34:80-94.

Maseko BC, Spocter MA, Haagensen M, Manger PR (2011): Volumetric analysis of the African elephant ventricular system. Anat Rec 294: 1412-1417.

Maseko BC, Spocter MA, Haagensen M, Manger PR (2012): Elephants have relatively the largest cerebellum size of mammals. Anat Rec 295:661-672.

-McCabe JT, Debellis M, Leibowitz SF (1984): Clonodine-induced feeding - analysis of central sites of action and fiber projections mediating this response. Brain Res 309:85-104. 
McGranaghan PA, Piggins HD (2001): Orexin Alike immunoreactivity in the hypothalamus and thalamus of the Syrian hamster (Mesocricetus auratus) and Siberain hamster (Phodopus sungorus), with special reference to circadian structures. Brain Res 904:234-244.

-McGregor R, Wu M-F, Barber G, Ramanthan L, Siegel JM (2011): Highly specific role of hypocretin (orexin) neurons: differential activation as a function of diurnal phase, operant reinforcement vs. operant avoidance and light level. J Neurosci 31:15455-15467.

- Meister B, Hökfelt T, Steinbusch HW, Skagerberg G, Lindvall O, Geffard M, Joh TH, Cuello AC, Goldstein M (1988): Do tyrosine hydroxylase-immunoreactive neurons in the ventrolateral arcuate nucleus produce dopamine or only L-dopa? J Chem Neuroanat 1:59-64.

Moon DJ, Maseko BC, Ihunwo AO, Fuxe K, Manger PR (2007): Distribution and morphology of catecholaminergic and serotonergic neurons in the brain of the highveld gerbil, Tatera brantsii. J Chem Neuroanat 34:134-144.

- Ngwenya A, Patzke N, Ihunwo AO, Manger PR (2011): Organisation and chemical neuroanatomy of the African elephant (Loxodonta africana) olfactory bulb. Brain Struct Funct 216:403-416.

-O'Connell-Rodwell CE, Wood JD, Kinzley C, Rodwell TC, Alarcon C, Wasser SK, Sapolsky R (2011): Male African elephants (Loxodonta africana) queue when the stakes are high. Ethol Ecol Evol 23:388-397.

O'Connell-Rodwell CE, Wood JD, Kinzley C, Rodwell TC, Poole JH, Puria S (2007): Wild African elephants (Loxodonta africana) discriminate between familiar and unfamiliar conspecific seismic alarm calls. J Acoust Soc Am 122:823-830.

-O'Connell-Rodwell CE, Wood JD, Rodwell TC, Puria S, Partan SR, Keefe R, Shriver D, Arnason BT, Hart LA (2006): Wild elephant (Loxodonta africana) breeding herds respond to artificially transmitted seismic stimuli. Behav Ecol Sociobiol 59:842-850.

Olson L, Fuxe K (1972): Further mapping out of the central noradrenaline neurons systems: projections of the 'subcoeruleus' area. Brain Res 43:289-295.

Paxinos G, Watson C, Carrive P, Kirkcaldie MTK, Ashwell K (2009): Chemoarchitectonic Atlas of the Rat Brain. New York, Elsevier.
Payne KB, Langbauer WE, Thomas EM (1986): Infrasonic calls of the Asian elephant (Elephas maximus). Behav Ecol Sociobiol 18:297-301.

- Peyron C, Tighe DK, van den Pol AN, de Lecea L, Heller HC, Sutcliffe JG, Kilduff TS (1998): Neurons containing hypocretin (orexin) project to multiple neuronal systems. J Neurosci 18:9996-10015.

Pieters RP, Gravett N, Fuxe K, Manger PR (2010): Nuclear organization of cholinergic, putative catecholaminergic and serotonergic nuclei in the brain of the eastern rock elephant shrew, Elephantulus myurus. J Chem Neuroanat 39: 175-188.

Pioli EY, Meissner W, Sohr R, Gross CE, Bezard E, Bioulac BH (2008): Differential behavioral effects of partial bilateral lesions of ventral tegmental area or substantia nigra pars compacta in rats. Neuroscience 153:1213-1224.

Precechtel A (1925): Some notes upon the finer anatomy of the brain stem and basal ganglia of Elephas indicus. Proc R Acad Sci Amsterdam 28:81-92.

Rasmussen LEL, Munger BL (1996): The sensorineural specializations of the trunk tip (finger) of the Asian elephant, Elephas maximus. Anat Rec 246:127-134.

Ridgway SH (1990): The central nervous system of the bottlenose dolphin; in Leatherwood S, Reeves RR (eds): The Bottlenose Dolphin. New York, Academic Press, pp 69-97.

-Ruggiero DA, Anwar M, Gootman PM (1992): Presumptive adrenergic neurons containing phenylethanolamine $\mathrm{N}$-methyltransferase immunoreactivity in the medulla oblongata of neonatal swine. Brain Res 583:105-119.

Shoshani J, Kupsky WJ, Marchant GH (2006): Elephant brain. 1. Gross morphology, functions, comparative anatomy, and evolution. Brain Res Bull 70:124-157.

Skinner JD, Chimimba CT (2005): The Mammals of the Southern African Subregion, ed 3. Cape Town, Cambridge University Press.

Smeets WJAJ, González A (2000): Catecholamine systems in the brain of vertebrates: new perspectives through a comparative approach. Brain Res Rev 33:308-379.

Smith F (1890): Histology of the skin of the elephant. J Anat Physiol 24:493-503.
Spinazzi R, Andries PG, Rossi GP, Nussdorfer GG (2006): Orexins in the regulation of the hypothalamic-pituitary-adrenal axis. Pharmacol Rev 58:46-57.

Steinbusch HWM (1981): Distribution of serotonin-immunoreactivity in the central nervous system of the rat - cell bodies and terminals. Neuroscience 6:557-618.

Stephan H, Frahm H, Baron G (1981): New and revised data on volumes of brain structures in insectivores and primates. Folia Primatol 35: $1-29$.

Sutcliffe JG, de Lecea L (2000): The hypocretins: excitatory neuromodulatory peptides for multiple homeostatic systems, including sleep and feeding. J Neurosci Res 62:161-168.

Tabuce R, Asher RJ, Lehmann T (2008): Afrotherian mammals: a review of current data. Mammalia 72:2-14.

Tobler I (1992): Behavioral sleep in the Asian elephant in captivity. Sleep 15:1-12.

Törk I (1990): Anatomy of the serotonergic system. Ann NY Acad Sci 600:9-35.

Tsuchitani C, Boudreau JC (1967): Encoding of stimulus frequency and intensity by cat superior olive S-segment cells. J Acoust Soc Am 42: 794-805.

Verhaart WJC (1962): Anatomy of the brain stem of the elephant. J Hirnforsch 5:455-525.

Verhaart WJC (1963): Pyramidal tract in the cord of the elephant. J Comp Neurol 121:45-50.

Verhaart WJC, Kramer W (1958): The pyramid in the medulla and the cord of the elephant. Acta Morphol Neerl Scand 2:174-182.

-Wagner D, Salin-Pascual R, Greco MA, Shiromani PJ (2000): Distribution of hypocretincontaining neurons in the lateral hypothalamus and $\mathrm{C}$-fos-immunoreactive neurons in the VLPO. Sleep Res Online 3:35-42.

Weihe E, Depboylu C, Schultz B, Schafer MKH, Edien LE (2006): Three types of tyrosine hydroxylase-positive CNS neurons distinguished by dopa decarboxylase and VMAT2 co-expression. Cell Mol Neurobiol 26:659678.

-Woolf NJ (1991): Cholinergic systems in mammalian brain and spinal cord. Prog Neurobiol 37:475-524.

Zhang SP, Davis PJ, Bandler R, Carrive P (1994): Brain stem integration of vocalization: role of the midbrain periaqueductal gray. J Neurophysiol 72:1337-1356. 